TRANSACTIONS OF THE

AMERICAN MATHEMATICAL SOCIETY

Volume 357 , Number 12, Pages 4897-4955

S 0002-9947(04)03618-9

Article electronically published on December 28, 2004

\title{
A GIESEKER TYPE DEGENERATION OF MODULI STACKS OF VECTOR BUNDLES ON CURVES
}

\author{
IVAN KAUSZ
}

\begin{abstract}
We construct a new degeneration of the moduli stack of vector bundles over a smooth curve when the curve degenerates to a singular curve which is irreducible with one double point. We prove that the total space of the degeneration is smooth and its special fibre is a divisor with normal crossings. Furthermore, we give a precise description of how the normalization of the special fibre of the degeneration is related to the moduli space of vector bundles over the desingularized curve.
\end{abstract}

\section{INTRODUCTION}

In this paper we construct a degeneration with nice properties of the moduli stack of vector bundles on a smooth curve when the curve degenerates to a singular curve which is irreducible with one double point.

Degeneration is a well-known technique in the study of moduli spaces of bundles on curves. The technique can be described as follows: Suppose one is interested in some invariant $\mathcal{N}$ of the moduli space $U(X)$ of (semistable) vector bundles (say of fixed rank and degree) on a smooth curve $X$. Suppose furthermore that the invariant does not really depend on the curve, but only on its genus $g$. Then it is often useful to consider a proper flat family of genus $g$ curves over a one-dimensional connected base, say $\mathcal{X} \rightarrow Y$, which is smooth over the complement of a point $y_{0} \in Y$ and whose fibre $\mathcal{X}_{y_{0}}$ over $y_{0}$ is singular. In addition one requires that the genus of the normalization $\widetilde{\mathcal{X}}_{y_{0}}$ of $\mathcal{X}_{y_{0}}$ is strictly smaller than $g$. If $g \geq 1$, one can always find such a family. More specifically, there exists a family such that $\mathcal{X}_{y_{0}}$ is irreducible with one double point (and thus the genus of $\widetilde{\mathcal{X}}_{y_{0}}$ is $g-1$ ). Now one tries to construct a proper flat family $U(\mathcal{X} / Y) \rightarrow Y$ of varieties such that:

(1) For every $y \in Y \backslash\left\{y_{0}\right\}$ the fibre $U(\mathcal{X} / Y)_{y}$ is isomorphic to $U\left(\mathcal{X}_{y}\right)$.

(2) The invariant $\mathcal{N}$ makes sense and takes the same value for all fibres $U(\mathcal{X} / Y)_{y}$.

(3) The varieties $U(\mathcal{X} / Y)_{y_{0}}$ and $U\left(\widetilde{\mathcal{X}}_{y_{0}}\right)$ are related in a way which enables one to compute the invariant for $U(\mathcal{X} / Y)_{y_{0}}$ in terms of the invariant for $U\left(\widetilde{\mathcal{X}}_{y_{0}}\right)$.

By this strategy one is finally reduced to the genus zero case where often one can determine the invariant directly.

Received by the editors January 28, 2004.

2000 Mathematics Subject Classification. Primary 14H60; Secondary 14D06, 14A20.

(C) 2004 by the author 
I know of two examples where this technique has been employed successfully:

One example is Sun's proof of the so-called "factorization rule". In this example, $U(X)$ is the space of semistable (parabolic) bundles (of given rank and degree) on a smooth (pointed) curve $X$ and the invariant $\mathcal{N}$ is the dimension of the space of global sections of (some power of) the generalized theta line bundle $\Theta$ on $U(X)$. In his proof Sun extends ideas of Narasimhan and Ramadas (cf. [NR]) who did the rank two case. For the degeneration technique he uses the family $U^{\text {tfs }}(\mathcal{X} / Y) \rightarrow Y$ of moduli spaces of torsion free sheaves as studied e.g. in $[\mathrm{N}]$ and $[\mathrm{Se} 1$. A variant of Bhosle's concept of generalized parabolic sheaves (cf. Bho]) allows him to relate $U^{\mathrm{tfs}}(\mathcal{X} / Y)_{y_{0}}$ with $U\left(\widetilde{\mathcal{X}}_{y_{0}}\right)$. Unfortunately his proof that $\mathcal{N}$ is independent of $y \in Y$ only works for $g \geq 3$. Therefore he cannot quite reduce to the genus zero case and his result does not lead to a formula for $\mathcal{N}$.

The other example for a successful employment of the degeneration technique is Gieseker's proof of a conjecture of Newstead and Ramanan. In this example $U(X)$ is the space of stable vector bundles of rank 2 and odd degree on a smooth curve $X$ of genus $g$ and $\mathcal{N}$ is the $m$-th Chern class of the tangent bundle of $U(X)$, where $m>2 g-2$. The conjecture of Newstead and Ramanan says that $\mathcal{N}=0$. For the degeneration technique, Gieseker uses a different family of moduli spaces than Sun: The fibre over $y_{0}$ of Gieseker's family $U^{\text {Gieseker }}(\mathcal{X} / Y) \rightarrow Y$ is a moduli space $U^{\text {Gieseker }}\left(\mathcal{X}_{y_{0}}\right)$ for certain rank 2 vector bundles. These vector bundles live either on $\mathcal{X}_{y_{0}}$ itself or on modifications $\mathcal{X}_{y_{0}}^{\prime}$ or $\mathcal{X}_{y_{0}}^{\prime \prime}$ of $\mathcal{X}_{y_{0}}$ which may be depicted as follows:
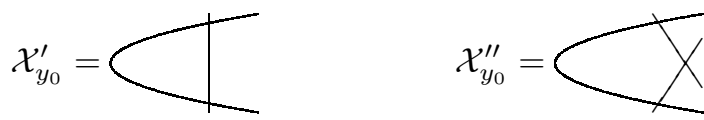

In these figures the straight lines stand for copies of the projective line $\mathbb{P}^{1}$ and the crooked ones stand for the normalization $\widetilde{\mathcal{X}}_{y_{0}}$ of $\mathcal{X}_{y_{0}}$. (As explained in the introduction of [K1] the space of vector bundles on $\mathcal{X}_{y_{0}}$ alone cannot be proper, so a compactification of that space must parametrize additional objects.) The relationship between $U^{\text {Gieseker }}\left(\mathcal{X}_{y_{0}}\right)$ and $U\left(\widetilde{\mathcal{X}}_{y_{0}}\right)$ is given by a diagram as follows:

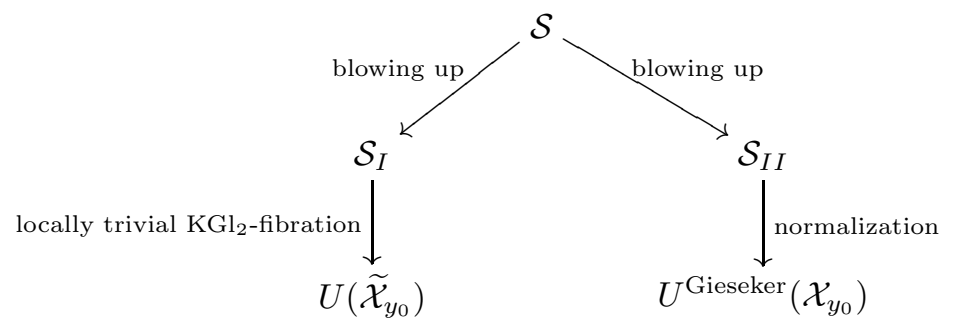

Here, $\mathrm{KGl}_{2}$ is a certain compactification of the general linear group $\mathrm{Gl}_{2}$. It is crucial for Gieseker's purpose that the variety $U^{\text {Gieseker }}(\mathcal{X} / Y)$ is regular and that the fibre of $U^{\text {Gieseker }}(\mathcal{X} / Y)$ over $y_{0}$ is a divisor with normal crossings, properties which are not shared by the family $U^{\mathrm{tfs}}(\mathcal{X} / Y) \rightarrow Y$.

Although expected by the experts (cf. introduction of $[\mathrm{G}]$ and $[\mathrm{T}]$ ), a higher rank generalization of Gieseker's family $U^{\text {Gieseker }}(\mathcal{X} / Y) \rightarrow Y$ was missing for a long time. Only quite recently Nagaraj and Seshadri constructed such a family in the arbitrary rank $n$ and coprime degree $d$ case (cf. [NS] and [Se2]). The fibre over $y_{0}$ of that family is a moduli space $U^{\text {Gieseker }}\left(\mathcal{X}_{y_{0}}\right)$ for what we call stable Gieseker vector bundles on $\mathcal{X}_{y_{0}}$. A Gieseker vector bundle on $\mathcal{X}_{y_{0}}$ is a vector bundle $\mathcal{E}$ on a 
modification $\mathcal{X}_{y_{0}}^{(r)}$ of $\mathcal{X}_{y_{0}}$ of the form

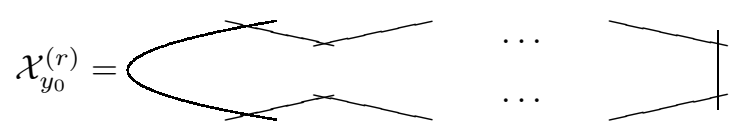

(the number $r \geq 0$ is the length of the inserted chain of projective lines, for $r=0$ we set $\mathcal{X}_{y_{0}}^{(0)}:=\mathcal{X}_{y_{0}}$ ) with the following properties:

(1) The restriction of $\mathcal{E}$ to any of the inserted projective lines is strictly standard, i.e. of the form $\mathcal{O}(1)^{\delta} \oplus \mathcal{O}^{n-\delta}$ for some $\delta>0$ which may depend on the projective line.

(2) The push forward $f_{*} \mathcal{E}$ of $\mathcal{E}$ by the canonical morphism $f: \mathcal{X}_{y_{0}}^{(r)} \rightarrow \mathcal{X}_{y_{0}}$ (which contracts the chain of projective lines to the singular point of $\mathcal{X}_{y_{0}}$ ) is torsion-free.

Such a Gieseker vector bundle $\mathcal{E}$ is called stable, if in (2) the sheaf $f_{*} \mathcal{E}$ is a stable torsion-free sheaf.

Nagaraj and Seshadri show that the total space $U^{\text {Gieseker }}(\mathcal{X} / Y)$ of their family is a regular projective variety and that the fibre over $y_{0}$ is a divisor with normal crossings. Furthermore they construct a canonical proper birational $Y$-morphism $U^{\text {Gieseker }}(\mathcal{X} / Y) \rightarrow U^{\mathrm{tfs}}(\mathcal{X} / Y)$. On the other hand they do not investigate the relationship between $U^{\text {Gieseker }}\left(\mathcal{X}_{y_{0}}\right)$ and $U\left(\widetilde{\mathcal{X}}_{y_{0}}\right)$, i.e. the higher rank analogue of diagram $(*)$. In particular they do not give a candidate for the compactification of $\mathrm{Gl}_{n}$, which should be the higher rank generalization for $\mathrm{KGl}_{2}$.

In the quest for a geometric proof of the Verlinde formula (cf. [So] ) and about the same time as Nagaraj and Seshadri, I arrived independently at a notion of (families of) rank $n$ Gieseker vector bundles on an irreducible stable curve $C_{0}$ (over some field $k$ ) with one singularity (parmetrized by a $k$-scheme $S$ ) and announced (in [K1) a result which assures that the associated moduli functor $\mathrm{GVB}_{n}\left(C_{0} / k\right)$ fits into a diagram

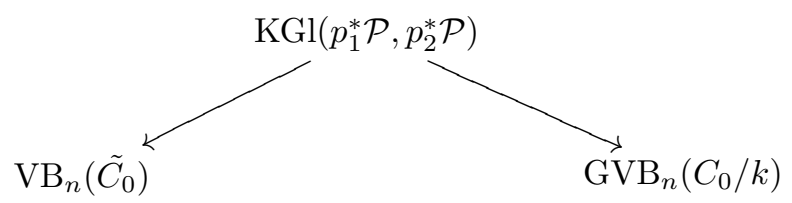

where $\operatorname{VB}_{n}\left(\tilde{C}_{0}\right)$ is the moduli functor of all - not just semistable - rank $n$ vector bundles on the normalization $\tilde{C}_{0}$ of $C_{0}$ and $\operatorname{KGl}\left(p_{1}^{*} \mathcal{P}, p_{2}^{*} \mathcal{P}\right) \rightarrow \operatorname{VB}_{n}\left(\tilde{C}_{0}\right)$ is a locally trivial fibre bundle with standard fibre the canonical compactification $\mathrm{KGl}_{n}$ of $\mathrm{Gl}_{n}$ as defined in $\mathrm{K} 1$.

In the present paper we prove more than that. Here is the precise statement. Let $B$ be the spectrum of a discrete valuation ring, $\operatorname{Spec}(k) \cong B_{0} \hookrightarrow B$ its special and $B_{1} \hookrightarrow B$ its generic point. We assume that $k$ is algebraically closed and of characteristic zero. Let $C \rightarrow B$ be a stable curve over $B$, whose generic fibre $C_{1}$ is smooth and whose special fibre $C_{0}$ is irreducible with one singularity $p \in C_{0}$. We assume furthermore that $C$ is regular. Let $n \geq 1$ and let $\operatorname{VB}_{n}\left(C_{1} / B_{1}\right)$ be the algebraic $B_{1}$-stack of vector bundles of rank $n$ over $C_{1}$. We define the notion of (families of) Gieseker vector bundles on $C$ and prove that the corresponding moduli problem is representable by an algebraic $B$-stack $\operatorname{GVB}_{n}(C / B)$ with the following 
properties:

(1) The stack $\operatorname{GVB}_{n}(C / B)$ is regular, locally of finite type and flat over $B$. Its special fibre $\operatorname{GVB}_{n}\left(C_{0} / B_{0}\right)$ is a divisor with normal crossings (Theorem 3.12).

(2) The generic fibre of $\mathrm{GVB}_{n}(C / B)$ is the moduli stack $\operatorname{VB}_{n}\left(C_{1} / B_{1}\right)$.

(3) In the diagram $(* *)$ the arrows are morphisms of $B_{0}$-stacks. $\operatorname{KGl}\left(p_{1}^{*} \mathcal{P}, p_{2}^{*} \mathcal{P}\right)$ is the fibre bundle over $\operatorname{VB}_{n}\left(\tilde{C}_{0}\right)$ associated as in [K1], Theorem 9.1, to the two vector bundles $p_{i}^{*} \mathcal{P}(i=1,2)$ which are the pull-back of the Poincaré bundle on $\tilde{C}_{0} \times \operatorname{VB}_{n}\left(\tilde{C}_{0}\right)$ by the sections given by the points $p_{1}, p_{2} \in \tilde{C}_{0}$ lying above $p$. The morphism $\operatorname{KGl}\left(p_{1}^{*} \mathcal{P}, p_{2}^{*} \mathcal{P}\right) \rightarrow \operatorname{GVB}_{n}\left(C_{0} / k\right)$ identifies $\operatorname{KGl}\left(p_{1}^{*} \mathcal{P}, p_{2}^{*} \mathcal{P}\right)$ with the normalization of $\mathrm{GVB}_{n}\left(C_{0} / k\right)$ (Theorems 4.9 and 9.5).

In a forthcoming paper (cf. [K2]) I will show how the third property can be used to prove a canonical direct sum decomposition of the space of global sections of a power of the theta line bundle on $\mathrm{GVB}_{n}\left(C_{0} / k\right)$.

There is also a stack version of the torsion-free sheaves approach: For an arbitrary base scheme $B$ and any semistable curve $C \rightarrow B$ there is an algebraic $B$-stack $\operatorname{TFS}_{n}(C / B)$ parametrizing relatively torsion free sheaves of rank $n$ on $C / B$. However the stack $\operatorname{TFS}_{n}(C / B)$ is not regular if $C \rightarrow B$ is not smooth. The singularities of $\operatorname{TFS}_{n}(C / B)$ have been studied by Faltings in $[\mathrm{F}$. (That paper also contains an analogous treatment of the stacks of principal $G$-bundles for $G$ the symplectic or the orthogonal group). Returning to our case (i.e. where $B$ is the spectrum of a discrete valuation ring, $C \rightarrow B$ generically smooth with irreducible special fibre with only one singularity), we have a morphism $\operatorname{GVB}_{n}(C / B) \rightarrow \operatorname{TFS}_{n}(C / B)$ which is defined in the same way as the morphism $U^{\text {Gieseker }}(\mathcal{X} / Y) \rightarrow U^{\mathrm{tfs}}(\mathcal{X} / Y)$ of Nagaraj and Seshadri and which may be considered as a resolution of singularities of $\operatorname{TFS}_{n}(C / B)$. The analogue in the torsion-free sheaves approach of the morphism $\operatorname{KGl}\left(p_{1}^{*} \mathcal{P}, p_{2}^{*} \mathcal{P}\right) \rightarrow \operatorname{GVB}_{n}\left(C_{0} / B_{0}\right)$ is a morphism $\operatorname{Grass}_{n}\left(p_{1}^{*} \mathcal{P} \oplus p_{2}^{*} \mathcal{P}\right) \rightarrow$ $\operatorname{TFS}_{n}\left(C_{0} / B_{0}\right)$. We show (cf. Proposition 10.1) that there exists a commutative diagram

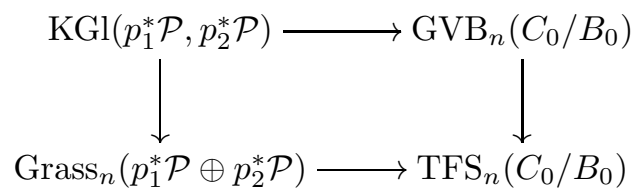

The left vertical arrow has been defined in [K1], where we have also computed the fibres of that morphism.

Our paper is organized as follows: In section 3 we define the notion of a Gieseker vector bundle on $C$ (resp. $C_{0}$ ) over a $B$-scheme (resp. a $k$-scheme) $S$. This is a pair $(\mathcal{C}, \mathcal{E})$, where $\mathcal{C}$ is a nodal curve over $\mathrm{S}$ which differs from the family $C \times{ }_{B} S$ (resp. $C_{0} \times_{k} S$ ) only by the replacement of some of its singular fibres by modifications $C_{0}^{(r)}$ of $C_{0}$ for some $r \leq n$, and where the restriction of $\mathcal{E}$ to any fibre of the form $C_{0}^{(r)}$ is "admissible" in the following sense:

(1) It is strictly standard.

(2) Its push forward by $C_{0}^{(r)} \rightarrow C_{0}$ is torsion free. 
(Actually our definition (cf. 3.6) is different, but equivalent to the above (cf. 3.7).) We then show that the associated moduli problem is represented by a regular algebraic $B$-stack $\operatorname{GVB}_{n}(C / B)$ and that its special fibre $\mathrm{GVB}_{n}\left(C_{0} / B_{0}\right)$ is a divisor with normal crossings. This section relies heavily on results in NS.

In section 4 an auxiliary notion is introduced: that of a Gieseker vector bundle data on $\left(\tilde{C}_{0}, p_{1}, p_{2}\right)$ over a $k$-scheme $S$. For $S=\operatorname{Spec}(k)$ this is just an admissible bundle on $C_{0}^{(r)}$ for some $r \geq 0$ plus the specification of a singular point in $C_{0}^{(r)}$. The associated moduli problem is represented by an algebraic $B_{0}$-stack $\mathrm{GVBD}_{n}\left(\tilde{C}_{0}, p_{1}, p_{2}\right)$ which we show to be canonically isomorphic to the normalization of $\operatorname{GVB}_{n}\left(C_{0} / B_{0}\right)$. Also in section 4 we use results from [NS].

Sections 5 through 9 are concerned with proving that $\operatorname{GVBD}_{n}\left(\tilde{C}_{0}, p_{1}, p_{2}\right)$ is a locally trivial $\mathrm{KGl}_{n}$-fibration over $\mathrm{VB}_{n}\left(\tilde{C}_{0} / B_{0}\right)$. For this, we show that a Gieseker vector bundle data on $\left(\tilde{C}_{0}, p_{1}, p_{2}\right)$ over a $k$-scheme $S$ uniquely corresponds to a vector bundle $\mathcal{E}$ over $\tilde{C}_{0} \times_{k} S$ together with a generalized isomorphism (in the sense of [K1], 5.2) from $p_{1}^{*} \mathcal{E}$ to $p_{2}^{*} \mathcal{E}$. By definition, a generalized isomorphism is made up of what we call bf-morphisms. A bf-morphism between vector bundles $E$ and $F$ over $S$ consists of

- a pair $(L, \lambda)$, where $L$ is an invertible $\mathcal{O}_{S}$-module and $\lambda$ is a global section of $L$, and

- a diagram $E \leftrightarrows \otimes \rightrightarrows F$ with certain properties, where the arrow $F \longrightarrow \otimes \rightarrow E$ means a morphism $F \rightarrow L \otimes E$ (cf. [K1], 5.1).

Our strategy is to break up Gieseker vector bundle data into simpler constituents and then to show a one-to-one correspondence between these constituents and bfmorphisms.

In section 5 we show that for a given curve $\pi: \mathcal{C} \rightarrow S$ together with a section $s$ of $\pi$ meeting $\mathcal{C}$ in the smooth locus, giving data $(L, \lambda)$ is equivalent to giving a new curve $\mathcal{C}^{\prime} \rightarrow S$ (together with a section $s^{\prime}$ ) which differs from $\mathcal{C}$ only by that over points $z \in S$ where $\lambda$ vanishes, a projective line is inserted at $s(z)$. We call $\left(\mathcal{C}^{\prime}, s^{\prime}\right)$ the simple modification of $(\mathcal{C}, s)$ associated to $(L, \lambda)$.

In section 6 we prove a technical lemma needed in section 5

In section 7 we define the notion of an admissible bundle over a simple modification. Then we show that given a curve $\pi: \mathcal{C} \rightarrow S$, a section $s$ of $\pi$ and a vector bundle $\mathcal{E}$ over $\mathcal{C}$ the data of a bf-morphism $\left(L, \lambda, E^{\prime} \leftrightarrows \otimes \square E=s^{*} \mathcal{E}\right)$ is equivalent to the data consisting of a simple modification $\left(\mathcal{C}^{\prime}, s^{\prime}\right)$ of $(\mathcal{C}, s)$ together with an admissible bundle $\mathcal{E}^{\prime}$ over $\mathcal{C}^{\prime}$ which satisfies certain properties relative to $\mathcal{E}$ and $E^{\prime}$.

In section 8 we prove that a Gieseker vector bundle data gives rise to a sequence of simple modifications together with admissible bundles on these.

Finally, in section 9 we put together the results from sections 5.8, to prove that indeed Gieseker vector bundle data correspond to generalized isomorphisms.

In the last section we establish the commutative diagram which clarifies the relationship of our construction with the torsion-free sheaves approach.

A remark on the restrictions on $k$ : Probably the results go through without assuming algebraic closedness and characteristic zero. Indeed the results in sections 5, [10 hold for general $k$. I imposed the restrictions on $k$ for the main results because I wanted to be able to cite theorems from $[\mathrm{NS}$ in sections 3 and 4 .

During the preparation of this paper I had the opportunity to spend six weeks at the Chennai Mathematical Institute. I would like to thank V. Balaji, D.S. Nagaraj 
and C.S. Seshadri for their interest in my work and all the other members of CMI for providing a friendly atmosphere.

\section{Notation}

Here we collect some notation which will be used freely in this paper.

- Throughout the article, $B$ denotes the spectrum of a discrete valuation ring, $\operatorname{Spec}(k) \cong B_{0} \hookrightarrow B$ its special and $B_{1} \hookrightarrow B$ its generic point. Furthermore, we fix once and for all a stable curve $C / B$ with smooth generic fibre $C_{1}$ and irreducible special fibre $C_{0}$ with only one singularity $p \in C_{0}$. We assume that $C$ is regular and that $\widehat{\mathcal{O}_{C_{0}, p}} \cong k[[u, v]] /(u . v)$. The symbol $\tilde{C}_{0}$ denotes the normalization of $C_{0}$ and $p_{1}, p_{2} \in \tilde{C}_{0}$ are the points which map to the singular point $p \in C_{0}$.

- Let $X$ be a scheme, $x$ a point of $X$ and $\mathcal{F}$ a coherent $\mathcal{O}_{X}$-module. Then we denote by $\mathcal{F}[x]$ the fibre $\mathcal{F}_{x} \otimes_{\mathcal{O}_{X, x}} \kappa(x)$ of $\mathcal{F}$ at $x$.

- For two integers $a, b$ the symbol $[a, b]$ denotes the set of integers $c$ with $a \leq c \leq b$.

- Let $S$ be a scheme. Let $\pi: X \rightarrow S$ be a prestable curve over $S$ (i.e. $\pi$ is flat, proper, such that the geometric fibres are reduced curves with at most ordinary double points). Let $Y$ be the disjoint union of finitely many copies of $S$ and for $\nu=1,2$ let $i_{\nu}: Y \rightarrow X$ be closed immersions such that

(1) The morphisms $i_{\nu}$ are $S$-morphisms.

(2) The subschemes $i_{1}(Y)$ and $i_{2}(Y)$ of $X$ intersect trivially.

(3) For $\nu=1,2$ the subscheme $i_{\nu}(Y)$ is contained in the smooth locus of $\pi$.

By $\mathrm{Kn}$, Theorem 3.4, there exists a prestable curve $X^{\prime} / S$ and an $S$ morphism $g: X \rightarrow X^{\prime}$, uniquely defined by the following properties:

(1) $g \circ i_{1}=g \circ i_{2}$ and $g$ is universal with this property.

(2) As a topological space, $X^{\prime}$ is the quotient of $X$ under the equivalence relation $i_{1}(y) \sim i_{2}(y)$ for $y \in Y$.

(3) For $U^{\prime}$ an open subset of $X^{\prime}$ and $U:=g^{-1}\left(U^{\prime}\right)$ the equality $\Gamma\left(U^{\prime}, \mathcal{O}_{X^{\prime}}\right)$ $=\left\{f \in \Gamma\left(U, \mathcal{O}_{X}\right) \mid i_{1}^{*} f=i_{2}^{*} f\right\}$ holds.

We refer to $X^{\prime}$ as the cokernel of the double arrow $Y \underset{i_{2}}{\stackrel{i_{1}}{\rightleftarrows}} X$.

- An algebraic stack will always be understood in the sense of [LM], (4.1).

\section{Gieseker vector Bundles}

Definition 3.1. Let $K$ be a field. A chain of rational curves over $K$ is a connected projective prestable curve whose associated graph is linear and whose irreducible components intersect in $K$-rational points and are each isomorphic to the rational line $\mathbb{P}_{K}^{1}$. The length of a chain of rational curves is the number of its irreducible components. We define a chain of length 0 of rational curves over $K$ to be just $\operatorname{Spec}(K)$.

Let $R$ be a chain of rational curves over $K$ of length $r \geq 1$ and let $R_{i} \subseteq R$ $(1 \leq i \leq r)$ be its irreducible components such that $R_{i} \cap R_{j} \neq \emptyset$ if and only if $|i-j| \leq 1$. For $1 \leq i \leq r-1$ let $x_{i} \in R(K)$ be the ordinary double point, in which the components $R_{i}$ and $R_{i+1}$ intersect. Furthermore, let $x_{0} \in R_{1}(K)$ and 
$x_{r} \in R_{r}(K)$ be smooth points with $x_{0} \neq x_{1}$. Thus the situation may be depicted as follows:

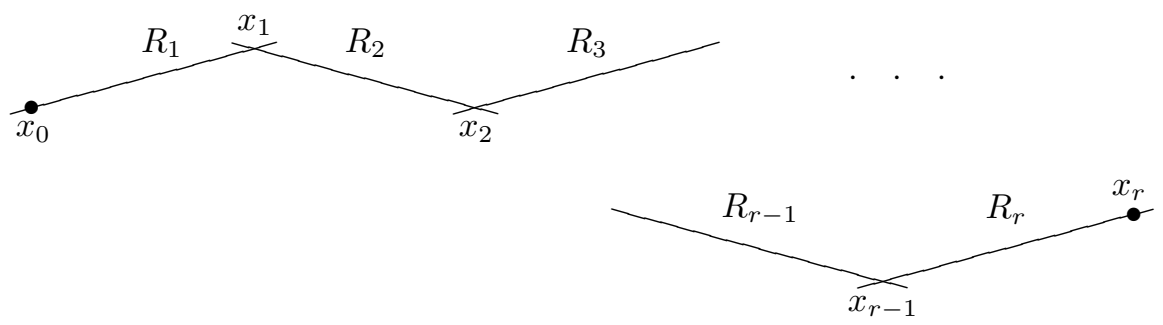

Let $\mathcal{E}$ be a locally free $\mathcal{O}_{R}$-module of rank $n$ such that for each $i$ one has

$$
\left.\mathcal{E}\right|_{R_{i}} \cong \bigoplus^{d_{i}} \mathcal{O}(1) \oplus \bigoplus^{n-d_{i}} \mathcal{O}
$$

with certain $d_{i} \in[1, n]$. Note that although the direct sum decomposition of $\left.\mathcal{E}\right|_{R_{i}}$ is not canonical, we have a canonical exact sequence

$$
\left.0 \rightarrow \mathcal{F}_{i} \rightarrow \mathcal{E}\right|_{R_{i}} \rightarrow \mathcal{G}_{i} \rightarrow 0
$$

such that $\mathcal{F}_{i} \cong \bigoplus^{d_{i}} \mathcal{O}(1)$ and $\mathcal{G}_{i} \cong \bigoplus^{n-d_{i}} \mathcal{O}$. Indeed, $\left(\left.\mathcal{F}_{i} \subseteq \mathcal{E}\right|_{R_{i}}\right)$ is the HarderNarasimhan filtration of $\left.\mathcal{E}\right|_{R_{i}}$. Therefore $\mathcal{E}$ induces a diagram of $K$-vector spaces

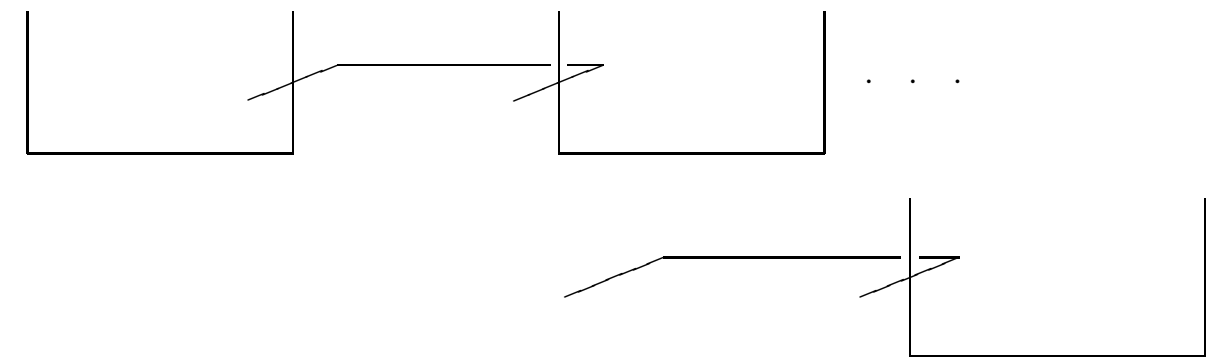

made up of $r$ horseshoes, where the $i$-th horseshoe stands for the diagram

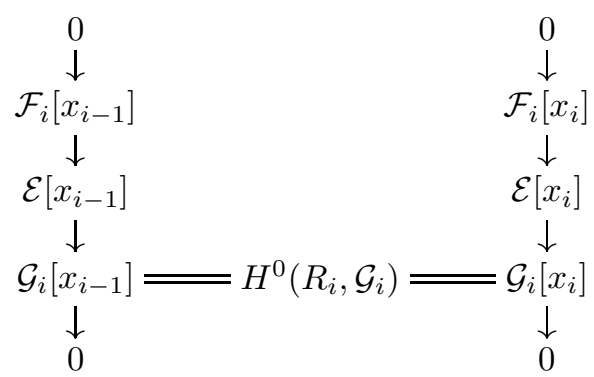

and the $i$-th and $(i+1)$-st horseshoe are linked together at $\mathcal{E}\left[x_{i}\right]$. We define subspaces $V_{i} \subseteq \mathcal{E}\left[x_{i}\right]$ for $0 \leq i \leq r$ inductively by setting $V_{0}:=(0)$ and $V_{i}:=$ (preimage under $\mathcal{E}\left[x_{i}\right] \rightarrow \mathcal{G}_{i}\left[x_{i}\right]$ of the image of $V_{i-1}$ under the morphism $\mathcal{E}\left[x_{i-1}\right] \rightarrow \mathcal{G}_{i}\left[x_{i-1}\right]=$ $\left.\mathcal{G}_{i}\left[x_{i}\right]\right)$.

Definition 3.2. Let $K$ be a field and let $R=\bigcup_{i=1}^{r} R_{i}$ be a chain of rational curves over $K$. A locally free $\mathcal{O}_{R}$-module of rank $n$ is called admissible, if either $R=\operatorname{Spec}(K)$ is of length zero, or $R$ is of length $r \geq 1$ with successive irreducible components $R_{1}, \ldots, R_{r}$ and the following two conditions hold. 
(1) $\mathcal{E}$ is strictly standard, i.e. for all $i$ the restriction of $\mathcal{E}$ to $R_{i}$ is isomorphic to $\bigoplus^{d_{i}} \mathcal{O}(1) \oplus \bigoplus^{n-d_{i}} \mathcal{O}$ for some $d_{i} \in[1, n]$.

(2) With the above notation, for all $i=1, \ldots, r-1$ the two subspaces $V_{i}$ and $\mathcal{F}_{i+1}\left[x_{i}\right]$ of $\mathcal{E}\left[x_{i}\right]$ intersect trivially.

Lemma 3.3. Let $K$ be a field, let $R=\bigcup_{i=1}^{r} R_{i}$ be a chain of length $r \geq 1$ of rational curves over $K$ and let $x_{0} \in R_{1}(K), x_{1} \in R_{r}(K)$ be two different smooth points on the extremal components. Let $\mathcal{E}$ be a strictly standard locally free $\mathcal{O}_{R}$-module of rank $n$. Then the following conditions are equivalent:

(1) $\mathcal{E}$ is admissible.

(2) $\operatorname{dim} H^{0}\left(R, \mathcal{E}\left(-x_{0}\right)\right)=\left.\sum_{i=1}^{r} \operatorname{deg} \mathcal{E}\right|_{R_{i}}$.

(3) $H^{0}\left(R, \mathcal{E}\left(-x_{0}-x_{r}\right)\right)=(0)$.

Furthermore, if one of these conditions holds, then for $i=1, \ldots, r$ the subspace $V_{i} \subseteq$ $\mathcal{E}\left[x_{i}\right]$ which intervenes in $\underline{3.2}(2)$ coincides with the image of $H^{0}\left(\left.\mathcal{E}\left(-x_{0}\right)\right|_{\bigcup_{j=1}^{i} R_{j}}\right)$ in $\mathcal{E}\left[x_{i}\right]$.

Proof. The equivalence of (2) and (3) has been shown in [NS], Lemma 2. Consider the following commutative diagram of epimorphisms of $K$-vector spaces:

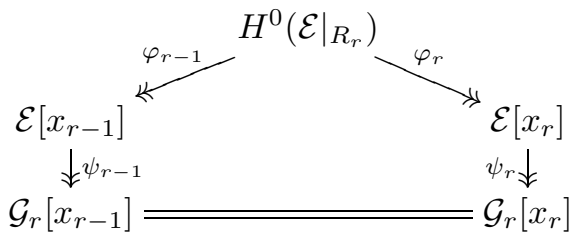

I claim that for any subspace $V \subseteq \mathcal{E}\left[x_{r-1}\right]$ we have $\varphi_{r}\left(\varphi_{r-1}^{-1}(V)\right)=\psi_{r}^{-1}\left(\psi_{r-1}(V)\right)$. Indeed, for this it is clearly sufficient to show that the kernel of $\psi_{r}$ is contained in $\varphi_{r}\left(\varphi_{r-1}^{-1}(V)\right)$. But we have $\operatorname{ker}\left(\varphi_{r-1}\right)=H^{0}\left(\left.\mathcal{E}\right|_{R_{r}}\left(-x_{r-1}\right)\right)=H^{0}\left(\mathcal{F}_{r}\left(-x_{r-1}\right)\right)$ and therefore $\operatorname{ker}\left(\psi_{r}\right)=\mathcal{F}_{r}\left[x_{r}\right]=\varphi_{r}\left(H^{0}\left(\mathcal{F}_{r}\left(-x_{r-1}\right)\right)\right) \subseteq \varphi_{r}\left(\varphi_{r-1}^{-1}(V)\right)$. We now show by induction on the length $r$ of $R$ that conditions (1) and (2) are equivalent and that the equality $V_{r}=\operatorname{im}\left(H^{0}\left(\mathcal{E}\left(-x_{0}\right)\right) \rightarrow \mathcal{E}\left[x_{r}\right]\right)$ holds. For $r=1$ conditions (1) and (2) are trivially equivalent and equality $V_{1}=\operatorname{im}\left(H^{0}\left(\mathcal{E}\left(-x_{0}\right)\right) \rightarrow \mathcal{E}\left[x_{1}\right]\right)$ follows from the above claim applied to $V=(0)$. If $r \geq 2$, we have

$$
H^{0}\left(\mathcal{E}\left(-x_{0}\right)\right)=H^{0}\left(\left.\mathcal{E}\left(-x_{0}\right)\right|_{\bigcup_{i=1}^{r-1} R_{i}}\right) \times_{\mathcal{E}\left[x_{r-1}\right]} H^{0}\left(\left.\mathcal{E}\right|_{R_{r}}\right) .
$$

Assuming condition (1) we clearly have $\operatorname{dim}\left(V_{r-1}\right)=\left.\sum_{i=1}^{r-1} \operatorname{deg} \mathcal{E}\right|_{R_{i}}$. By the induction hypothesis we therefore have $\operatorname{dim}\left(H^{0}\left(\left.\mathcal{E}\left(-x_{0}\right)\right|_{\bigcup_{i=1}^{r-1} R_{i}}\right)=\operatorname{dim}\left(V_{r-1}\right)\right.$ and furthermore $V_{r-1}=\operatorname{im}\left(H^{0}\left(\left.\mathcal{E}\left(-x_{0}\right)\right|_{\bigcup_{i=1}^{r-1} R_{i}}\right) \rightarrow \mathcal{E}\left[x_{r-1}\right]\right)$. This implies in particular that the canonical map from $H^{0}\left(\left.\mathcal{E}\left(-x_{0}\right)\right|_{\bigcup_{i=1}^{r-1} R_{i}}\right)$ to $\mathcal{E}\left[x_{r-1}\right]$ is injective. Therefore $H^{0}\left(\mathcal{E}\left(-x_{0}\right)\right)$ may be identified with the subspace $\varphi_{r-1}^{-1}\left(V_{r-1}\right)$ of $H^{0}\left(\left.\mathcal{E}\right|_{R_{r}}\right)$. It follows that $\operatorname{dim}\left(H^{0}\left(\mathcal{E}\left(-x_{0}\right)\right)\right)=\operatorname{dim}\left(V_{r-1}\right)+\operatorname{dim}\left(\operatorname{ker} \varphi_{r-1}\right)=\left.\sum_{i=1}^{r} \operatorname{deg} \mathcal{E}\right|_{R_{i}}$, i.e. $\mathcal{E}$ satisfies condition (2) and furthermore that we have

$$
\operatorname{im}\left(H^{0}\left(\mathcal{E}\left(-x_{0}\right)\right) \rightarrow \mathcal{E}\left[x_{r}\right]\right)=\varphi_{r}\left(\varphi_{r-1}^{-1}\left(V_{r-1}\right)\right)=\psi_{r}^{-1}\left(\psi_{r-1}\left(V_{r-1}\right)\right)=V_{r} \quad .
$$

Conversely, if $\mathcal{E}$ satisfies conditions (2) and (3), then the map $H^{0}\left(\left.\mathcal{E}\left(-x_{0}\right)\right|_{\bigcup_{i=1}^{r-1} R_{i}}\right) \rightarrow$ $\mathcal{E}\left[x_{r-1}\right]$ is injective, since its kernel is $H^{0}\left(\left.\mathcal{E}\left(-x_{0}-x_{r-1}\right)\right|_{\bigcup_{i=1}^{r-1} R_{i}}\right)=(0)$. As above, we conclude that $\operatorname{im}\left(H^{0}\left(\mathcal{E}\left(-x_{0}\right)\right) \rightarrow \mathcal{E}\left[x_{r}\right]\right)=V_{r}$. Since $H^{0}\left(\mathcal{E}\left(-x_{0}\right)\right) \rightarrow \mathcal{E}\left[x_{r}\right]$ is 
injective by condition (3), it follows that $\operatorname{dim} V_{r}=\left.\sum_{i=1}^{r} \operatorname{deg} \mathcal{E}\right|_{R_{i}}$ which is clearly equivalent to $\mathcal{E}$ being admissible.

Remark 3.4. Observe that admissibility of $\mathcal{E}$ implies that the length of $R$ is at most the rank of $\mathcal{E}$. From Lemma 3.3 it is easy to deduce the following statement: Let $R^{\prime}:=\bigcup_{i=1}^{r^{\prime}} R_{i}$ and $R^{\prime \prime}:=\bigcup_{i=r^{\prime}+1}^{r} R_{i}$ be subchains of $R$. If $\mathcal{E}$ is admissible, then $\left.\mathcal{E}\right|_{R^{\prime}}$ and $\left.\mathcal{E}\right|_{R^{\prime \prime}}$ are both admissible. Indeed, by 3.3 it suffices to show that $H^{0}\left(R^{\prime},\left.\mathcal{E}\right|_{R^{\prime}}\left(-x_{0}-x_{r^{\prime}}\right)\right)=(0)=H^{0}\left(R^{\prime \prime},\left.\mathcal{E}\right|_{R^{\prime \prime}}\left(-x_{r^{\prime}}-x_{r}\right)\right)$. Suppose that there exists a nontrivial section $\alpha^{\prime} \in H^{0}\left(R^{\prime},\left.E\right|_{R^{\prime}}\left(-x_{0}-x_{r^{\prime}}\right)\right)$. Then we can extend $\alpha^{\prime}$ by zero to obtain a nontrivial section $\alpha \in H^{0}\left(R, \mathcal{E}\left(-x_{0}-x_{r}\right)\right)$ which is impossible by assumption.

Definition 3.5. Let $K$ be a field and $X / K$ a curve. Let $x \in X(K)$ be a point with $\widehat{\mathcal{O}_{X, x}} \cong K[[u, v]] /(u v)$ and let $\tilde{X} \rightarrow X$ be the blowing-up of $X$ at $x$. Let $x^{\prime}, x^{\prime \prime} \in \tilde{X}(K)$ be the two points above $x$. A modification of $X$ at $x$ is a morphism $h: Y \rightarrow X$ such that

(1) $Y$ is isomorphic to the cokernel of the double arrow (cf. section 2)

$$
\operatorname{Spec}(K) \amalg \operatorname{Spec}(K) \underset{x_{0} \amalg x_{r}}{\stackrel{x^{\prime} \amalg x^{\prime \prime}}{\longrightarrow}} \tilde{X} \amalg R
$$

where $R$ is a chain of rational curves over $K$ of length $r$ and $x_{0}, x_{r}$ are smooth $K$-rational points of $R$, which lie in different extremal components if $r \geq 2$, are not equal if $r=1$, and are identical if $r=0$.

(2) The composed morphism $\tilde{X} \rightarrow Y \rightarrow X$ (resp. $R \rightarrow Y \rightarrow X$ ) is the blowingup morphism (resp. the constant morphism which maps $R$ onto $x$ ).

If $r=0$, then $Y=X$. For general $r$, a modification $Y$ looks like:

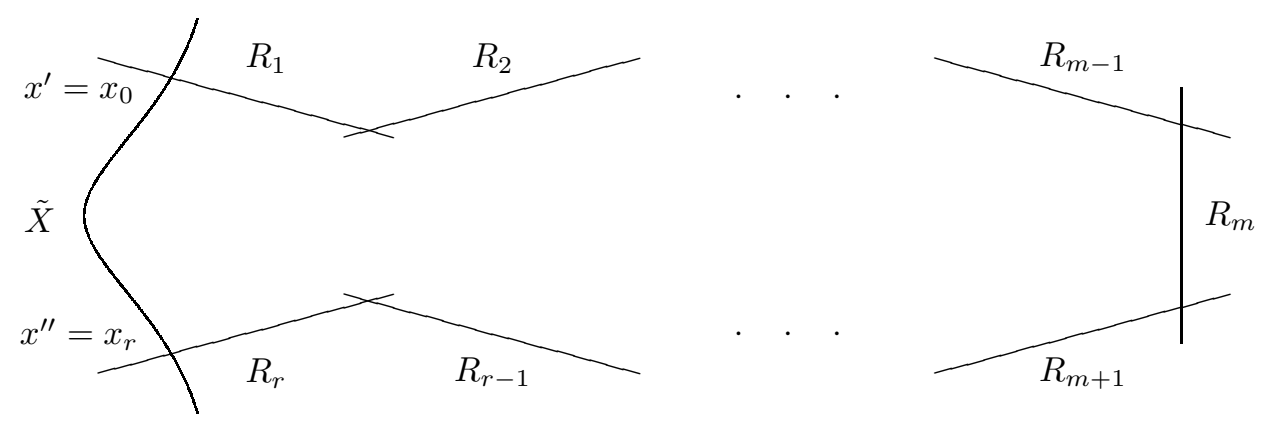

Definition 3.6. Let $X / K$ and $x \in X(K)$ be as in Definition 3.5 and let $h: Y \rightarrow X$ be a modification of $X$ at $x$. Let $R:=h^{-1}(x)$ be the chain of rational curves which is contracted to $x$. A locally free $\mathcal{O}_{Y}$-module $\mathcal{E}$ is called admissible for $Y \rightarrow X$ if its restriction to $R$ is admissible.

Lemma 3.7 ([NS, Prop. 5). With the notation of Definition 3.6 and assuming $X$ to be irreducible with only one double point, we have: A locally free $\mathcal{O}_{Y}$-module $\mathcal{E}$ is admissible for $Y \rightarrow X$ if and only if $\mathcal{E}$ is strictly positive and $h_{*} \mathcal{E}$ is torsion-free, where "strictly positive" means that the restriction of $\mathcal{E}$ to any component of $R$ is of the form $\bigoplus_{i} \mathcal{O}\left(a_{i}\right)$ for some $a_{i} \geq 0$ with $\sum_{i} a_{i} \neq 0$. 
Definition 3.8. Let $C \rightarrow B$ be as in section 2 ,

(1) Let $S$ be a $B$-scheme. A modification of $C$ over $S$ is a commutative diagram of schemes

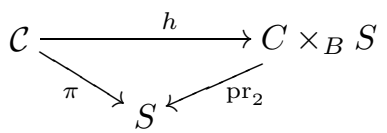

where

(a) $\pi$ is flat,

(b) $h$ is projective and finitely presented and induces an isomorphism $\mathcal{C} \times{ }_{S}$ $S_{1} \stackrel{\sim}{\rightarrow} C_{1} \times_{B_{1}} S_{1}$, where $S_{1}:=S \times_{B} B_{1}$, and

(c) for all $z \in S \times{ }_{B} B_{0}$ the induced morphism $\mathcal{C}_{z} \rightarrow C_{0} \times{ }_{B_{0}} \operatorname{Spec}(\kappa(z))$ on the fibres over $z$ is a modification (in the sense of 3.5 ) of the curve $C_{0} \times_{B_{0}} \operatorname{Spec}(\kappa(z))$ in the point $p \times_{B_{0}} \operatorname{Spec}(\kappa(z))$.

(2) Let $S$ be a $k$-scheme. A modification of $C_{0}$ over $S$ is a modification of $C$ over $S$, where $S$ is viewed as a $B$-scheme via $S \rightarrow \operatorname{Spec}(k)=B_{0} \hookrightarrow B$.

Lemma 3.9. Let $S$ be a B-scheme, let $h: \mathcal{C} \rightarrow C \times{ }_{B} S$ be a modification of $C$ over $S$ and let $\pi: \mathcal{C} \rightarrow S$ be the structure morphism. Then the natural morphism $\mathcal{O}_{S} \rightarrow \pi_{*} \mathcal{O}_{\mathcal{C}}$ is an isomorphism.

Proof. First we show that $h_{*} \mathcal{O}_{\mathcal{C}}=\mathcal{O}_{X \times_{B} S}$. By $\mathrm{Kn}$, Cor. 1.5, the $\mathcal{O}_{X \times_{B} S^{-}}$ module $h_{*} \mathcal{O}_{\mathcal{C}}$ is flat over $S$ and we have for all $z \in S$ the equality $h_{*} \mathcal{O}_{\mathcal{C}} \otimes_{\mathcal{O}_{S}}$ $\kappa(z)=\left(h_{z}\right)_{*} \mathcal{O}_{\mathcal{C}_{z}}$, where $h_{z}: \mathcal{C}_{z} \rightarrow X \times_{B} \operatorname{Spec}(\kappa(z))$ is the induced morphism between the fibres over $z$. Since obviously $\left(h_{z}\right)_{*} \mathcal{O}_{\mathcal{C}_{z}}=\mathcal{O}_{X \times{ }_{B} \operatorname{Spec}(\kappa(z))}$, it follows by Nakayama's lemma that we indeed have $h_{*} \mathcal{O}_{\mathcal{C}}=\mathcal{O}_{X \times_{B} S}$. Now consider the functor $T: M \mapsto H^{0}\left(C, \mathcal{O}_{C} \otimes_{\mathcal{O}_{B}} M\right)$ from the category of $\mathcal{O}_{B}$-modules into the category of $\mathcal{O}_{B}$-modules. Since $C \rightarrow B$ is a stable curve, we have $H^{0}\left(C_{b}, \mathcal{O}_{C_{b}}\right)=\kappa(b)$ for $b \in B$. Therefore $T$ is exact by [EGA III], (7.8.4). By EGA III], (7.7.5.3), it follows that $\left(\mathrm{pr}_{2}\right)_{*} \mathcal{O}_{C \times_{B} S}=H^{0}\left(C, \mathcal{O}_{C}\right) \otimes_{\mathcal{O}_{B}} \mathcal{O}_{S}$. Thus it suffices to show that $H^{0}\left(C, \mathcal{O}_{C}\right)=\mathcal{O}_{B}$. But this is clear: $H^{0}\left(C, \mathcal{O}_{C}\right)$ is a free $\mathcal{O}_{B}$-module by [EGA III, (7.8.4), and the morphism $\mathcal{O}_{B} \rightarrow H^{0}\left(C, \mathcal{O}_{C}\right)$ is an isomorphism by EGA III, (7.7.5)d).

Definition 3.10. If $\mathcal{C} \rightarrow C \times{ }_{B} S$ is a modification of $C$ over a $B$-scheme $S$ (cf. Definition (3.8), a locally free $\mathcal{O}_{\mathcal{C}}$-module $\mathcal{E}$ is called admissible for $\mathcal{C} \rightarrow C \times{ }_{B} S$, if for all $z \in S \times{ }_{B} B_{0}$ the restriction of $\mathcal{E}$ to the fibre $\mathcal{C}_{z}$ over $z$ is admissible for $\mathcal{C}_{z} \rightarrow C_{0} \times{ }_{B_{0}} \operatorname{Spec}(\kappa(z))$ in the sense of Definition 3.6.

Definition 3.11. Let $C \rightarrow B$ be as in section 2, Let $S$ be a $B$-scheme. A Gieseker vector bundle (of rank $n$ ) on $C$ over $S$ is a modification $\mathcal{C} \rightarrow C \times{ }_{B} S$ together with a locally free $\mathcal{O}_{\mathcal{C}}$-module $\mathcal{E}$ (of rank $n$ ), which is admissible for $\mathcal{C} \rightarrow C \times{ }_{B} S$. If $S$ is a $k$-scheme, a Gieseker vector bundle on $C_{0}$ over $S$ is a Gieseker vector bundle on $C$ over $S$, where $S$ is viewed as a $B$-scheme via $S \rightarrow \operatorname{Spec}(k)=B_{0} \hookrightarrow B$.

There is an obvious notion of an isomorphism between two Gieseker vector bundles on $C$ over a $B$-scheme $S$, and of pull-back of a Gieseker vector bundle under a morphism $S^{\prime} \rightarrow S$. Thus we have a functor:

$$
\operatorname{GVB}_{n}(C / B):\left\{\begin{array}{ccc}
\{B \text {-schemes }\}^{\circ} & \rightarrow & \text { \{groupoids }\} \\
S & \mapsto & \text { (Gieseker vector bundles } \\
& \text { of rank } n \text { on } C \text { over } S \text { ) }
\end{array}\right.
$$


Similarly, we have a functor:

$$
\operatorname{GVB}_{n}\left(C_{0} / k\right):\left\{\begin{array}{cll}
\{k \text {-schemes }\}^{o} & \rightarrow & \text { \{groupoids }\} \\
S & \mapsto \quad & \text { (Gieseker vector bundles } \\
& \text { of rank } n \text { on } C_{0} \text { over } S \text { ) }
\end{array}\right.
$$

Since we will always work with bundles of fixed rank, we will often suppress the index $n$ in the notation of these functors. We may also consider $\operatorname{GVB}\left(C_{0} / k\right)$ as a functor on $\{B$-schemes $\}$ by setting $\operatorname{GVB}\left(C_{0} / k\right)(S):=\operatorname{GVB}\left(C_{0} / k\right)(S \rightarrow \operatorname{Spec}(k))$, if the structure morphism $S \rightarrow B$ factorizes over $B_{0}=\operatorname{Spec}(k)$ and setting $\operatorname{GVB}\left(C_{0} / k\right)(S):=\emptyset$ else. In this sense we have the following cartesian diagram in the category of $B$-groupoids:

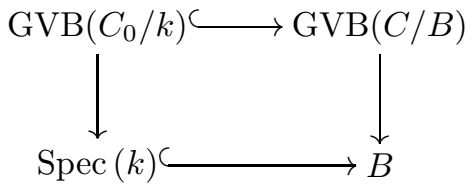

i.e. $\operatorname{GVB}\left(C_{0} / k\right)$ is the special fibre of $\operatorname{GVB}(C / B) \rightarrow B$.

Theorem 3.12. Assume that the residue field $k$ of $B$ is algebraically closed and of characteristic zero. Then the B-groupoid $G V B(C / B)$ is an algebraic stack which is locally of finite type and flat over $B$ and $G V B\left(C_{0} / k\right) \hookrightarrow G V B(C / B)$ is a divisor with normal crossings.

Proof. This follows from 3.16, 3.17, 3.21 and 3.24 below.

Lemma 3.13. Let $S$ be a locally noetherian scheme and let $f: X \rightarrow Y$ be an $S$-morphism of proper $S$-schemes. Let $s \in S$ be a point such that the morphism $f_{s}: X_{s} \rightarrow Y_{s}$ between the fibres over $s$ is a closed immersion. Then there is an open neighbourhood $S^{\prime} \subseteq S$ of s such that the induced morphism $X \times_{S} S^{\prime} \rightarrow Y \times_{S} S^{\prime}$ is a closed immersion.

Proof. (I suppose this is well known but I could not find a suitable reference.) The assumptions imply that the morphism $f$ is proper. By a theorem of Chevalley (cf. EGA IV], (13.1.3)) there is an open neighbourhood $Y^{\prime}$ of the fibre $Y_{s}$ such that all fibres of $X^{\prime}:=f^{-1}\left(Y^{\prime}\right) \rightarrow Y^{\prime}$ are 0-dimensional. By Zariski's main theorem the morphism $X^{\prime} \rightarrow Y^{\prime}$ is finite and by Nakayama's lemma the morphism $\mathcal{O}_{Y, y} \rightarrow$ $\left(f_{*} \mathcal{O}_{X}\right)_{y}$ is surjective for all $y \in Y_{s}$. Therefore there is an open neighbourhood $Y^{\prime \prime} \subseteq Y^{\prime}$ of $Y_{s}$ such that $\mathcal{O}_{Y^{\prime \prime}} \rightarrow f_{*}^{\prime \prime} \mathcal{O}_{X^{\prime \prime}}$ is surjective, where $X^{\prime \prime}:=f^{-1}\left(Y^{\prime \prime}\right)$ and $f^{\prime \prime}:=\left.f\right|_{X^{\prime \prime}}$. Let $q: Y \rightarrow S$ be the structure morphism and let $S^{\prime}:=S \backslash q\left(Y \backslash Y^{\prime \prime}\right)$. Then $S^{\prime}$ is an open neighbourhood of $s$ in $S$ with $Y \times_{S} S^{\prime} \subseteq Y^{\prime \prime}$. The morphism $f^{\prime}: X \times_{S} S^{\prime} \rightarrow Y \times_{S} S^{\prime}$ is finite and $\mathcal{O}_{Y \times{ }_{S} S^{\prime}} \rightarrow f_{*}^{\prime} \mathcal{O}_{X \times{ }_{S} S^{\prime}}$ is surjective. Thus $f^{\prime}$ is a closed immersion.

Lemma 3.14. Let $C \rightarrow B$ be as in section 2, Let $S$ be a locally noetherian $B$ scheme, $h: \mathcal{C} \rightarrow C \times{ }_{B} S$ a proper morphism such that $\pi:=p r_{2} \circ h$ is flat, and let $\mathcal{E}$ be a coherent $\mathcal{O}_{\mathcal{C}}$-module which is flat over $S$. Let $z \in S$ be a point such that the pair $\left(\mathcal{C}_{z} \rightarrow C \times_{B} \operatorname{Spec}(\kappa(z)) ;\left.\mathcal{E}\right|_{\mathcal{C}_{z}}\right)$ is a Gieseker vector bundle of rank $n$ on $C$ over Spec $(\kappa(z))$. Then there is an open neighbourhood $S^{o}$ of $z$ in $S$ such that $\left(\mathcal{C}^{o}:=\mathcal{C} \times{ }_{S} S^{o} \rightarrow C \times{ }_{B} S^{o} ;\left.\mathcal{E}\right|_{\mathcal{C}^{\circ}}\right)$ is a Gieseker vector bundle of rank $n$ on $C$ over $S^{o}$. 
Proof. As has been shown in NS (appendix), being a modification is an open condition. Thus there exists an open neighbourhood $U \subseteq S$ of $z$, such that $h_{U}$ : $\mathcal{C}_{U}:=\mathcal{C} \times{ }_{S} U \rightarrow C \times{ }_{B} U$ is a modification of $C$ over $U$. It is easy to see that after possibly shrinking $U$ we may also assume $\mathcal{E}_{U}:=\left.\mathcal{E}\right|_{U}$ to be locally free of rank $n$. We claim that if we choose $U$ small enough, we may even assume $\mathcal{E}_{U}$ to be admissible for $h_{U}$. By definition of a modification, $h_{U}$ is an isomorphism over the generic fibre of $U \rightarrow B$, so admissibility of $\mathcal{E}_{U}$ over points in the generic fibre is trivial. Thus we can restrict to the case where $U \rightarrow B$ factorizes over the closed point $B_{0}$ of $B$. Let $\mathcal{F}:=\left(h_{U}\right)_{*} \mathcal{E}_{U}$ and let $\mathcal{E}_{z}$ (resp. $\mathcal{F}_{z}$ ) be the restriction of $\mathcal{E}$ (resp. of $\mathcal{F}$ ) to the fibre $\mathcal{C}_{z}:=\mathcal{C} \times{ }_{S} \operatorname{Spec}(\kappa(z))$ (resp. to $\left.C \times{ }_{B} \operatorname{Spec}(\kappa(z))\right)$. By [Kn], Cor. 1.5, we have $\mathcal{F}_{z}=\left(h_{z}\right)_{*} \mathcal{E}_{z}$, where

$$
h_{z}: \mathcal{C}_{z}:=\mathcal{C} \times{ }_{S} \operatorname{Spec}(\kappa(z)) \rightarrow C \times{ }_{B} \operatorname{Spec}(\kappa(z))
$$

denotes the morphism between the fibres induced by $h$. Therefore Lemma 3.7 implies that $\mathcal{E}_{z}$ is strictly positive and that $\mathcal{F}_{z}$ is torsion free. We have to show that $\mathcal{E}_{z^{\prime}}$ being strictly positive and $\mathcal{F}_{z^{\prime}}$ being torsion free are open conditions on $z^{\prime} \in U$. Let $\mathcal{L}$ be a very ample invertible sheaf on $C_{0}:=C \times_{B} B_{0}$. Then the strict positivity of $\mathcal{E}_{z^{\prime}}$ is equivalent to the following: There exists an $N \in \mathbb{N}$ such that the sheaf $\mathcal{E}_{z^{\prime}}(N):=\mathcal{E}_{z^{\prime}} \otimes_{\mathcal{O}_{\mathcal{C}^{\prime}}} h_{z^{\prime}}^{*}\left(\mathcal{L}^{\otimes N} \otimes_{\mathcal{O}_{B}} \kappa\left(z^{\prime}\right)\right)$ has the properties (i) $H^{i}\left(\mathcal{C}_{z^{\prime}}, \mathcal{E}_{z^{\prime}}(N)\right)=0$ for $i \geq 1$, (ii) $\mathcal{E}_{z^{\prime}}(N)$ is generated by its global sections, and (iii) the morpism from $\mathcal{C}_{z^{\prime}}$ to the Grassmannian $\operatorname{Grass}_{n}\left(H^{0}\left(\mathcal{E}_{z^{\prime}}(N)\right)\right)$ induced by the surjection $H^{0}\left(\mathcal{E}_{z^{\prime}}(N)\right) \otimes_{\kappa\left(z^{\prime}\right)} \mathcal{O}_{\mathcal{C}_{z^{\prime}}} \rightarrow \mathcal{E}_{z^{\prime}}$ is a closed embedding. With this characterization and Lemma 3.13 it is easy to see that strict positivity is indeed an open condition on $z^{\prime}$. Since $C_{0}$ is irreducible, torsion freeness of $\mathcal{F}_{z^{\prime}}$ is equivalent to $\mathcal{F}_{z^{\prime}}$ being of depth one (cf. [Se1], p. 146, Lemma 2). By [EGA IV], (12.2.2), this is an open condition on $z^{\prime}$.

The following lemma will allow us in some proofs to restrict to the case of a noetherian base scheme.

Lemma 3.15. Let $C \rightarrow B$ be as in section 2 , Let $\left(S_{\lambda}\right)_{\lambda}$ be a projective system of affine $B$-schemes and let $S:=\lim S_{\lambda}$. Let $\left(\mathcal{C} \rightarrow C \times_{B} S ; \mathcal{E}\right)$ be a Gieseker vector bundle of rank $n$ on $C$ over $S$. Then there exists an index $\alpha$, an open subscheme $S^{\prime} \subset S_{\alpha}$ and a Gieseker vector bundle $\left(\mathcal{C}^{\prime} \rightarrow C \times{ }_{B} S^{\prime} ; \mathcal{E}^{\prime}\right)$ of rank $n$ on $C$ over $S^{\prime}$ such that the morphism $S \rightarrow S_{\alpha}$ factorizes through $S^{\prime}$ and $\left(\mathcal{C} \rightarrow C \times_{B} S ; \mathcal{E}\right)$ is induced by pull-back via $S \rightarrow S^{\prime}$ from $\left(\mathcal{C}^{\prime} \rightarrow C \times_{B} S^{\prime} ; \mathcal{E}^{\prime}\right)$.

Proof. By [EGA IV], (8.8.2)(ii), there exists an index $\lambda$ and an $S_{\lambda}$-scheme of finite type $\mathcal{C}_{\lambda}$ with $\mathcal{C} \cong \mathcal{C}_{\lambda} \times_{S_{\lambda}} S$. By EGA IV], (8.10.5)(xii) and (11.2.6)(ii), we may assume that $\mathcal{C}_{\lambda} \rightarrow S_{\lambda}$ is proper and flat. By EGA IV, (8.8.2)(i), (8.5.2) and (11.2.6)(ii), we may assume furthermore that the morphism $\mathcal{C} \rightarrow C \times{ }_{B} S$ is induced by base-change from a proper morphism $\mathcal{C}_{\lambda} \rightarrow C \times{ }_{B} S_{\lambda}$ and that $\mathcal{E}$ is the pull-back of a coherent $\mathcal{O}_{\mathcal{C}_{\lambda}}$-module $\mathcal{E}_{\lambda}$, flat over $S_{\lambda}$. It is easy to see that if $K / \kappa$ is a field extension, $\operatorname{Spec}(\kappa) \rightarrow B$ a morphism, $\mathcal{C}_{\kappa} \rightarrow C \times_{B} \operatorname{Spec}(\kappa)$ a proper morphism and $\mathcal{E}_{\kappa}$ a coherent $\mathcal{O}_{\mathcal{C}_{\kappa}}$-module such that the pull-back $\left(\mathcal{C}_{K} \rightarrow C \times_{B} \operatorname{Spec}(K) ; \mathcal{E}_{K}\right)$ of the data $\left(\mathcal{C}_{\kappa} \rightarrow C \times_{B} \operatorname{Spec}(\kappa) ; \mathcal{E}_{\kappa}\right)$ via $\operatorname{Spec}(K) \rightarrow \operatorname{Spec}(\kappa)$ is a Gieseker vector bundle on $C$ over $\operatorname{Spec}(K)$, then there is a finite sub-extension $\kappa^{\prime} / \kappa$ of $K / \kappa$, such that already the pull-back of $\left(\mathcal{C}_{\kappa} \rightarrow C \times{ }_{B} \operatorname{Spec}(\kappa) ; \mathcal{E}_{\kappa}\right)$ via $\operatorname{Spec}\left(\kappa^{\prime}\right) \rightarrow \operatorname{Spec}(\kappa)$ is a Gieseker vector bundle on $C$ over Spec $\left(\kappa^{\prime}\right)$. Therefore we can find for each $z \in S$ an index $\lambda_{z} \geq \lambda$, such that the pull-back of $\left(\mathcal{C}_{\lambda} \rightarrow C \times_{B} S_{\lambda} ; \mathcal{E}_{\lambda}\right)$ via $\operatorname{Spec}(\kappa(z)) \rightarrow S_{\lambda}$ 
is a Gieseker vector bundle, where $z^{\prime}$ denotes the image of $z$ in $S_{\lambda_{z}}$. By Lemma 3.14, there is an open neighbourhood $U_{z} \subseteq S_{\lambda_{z}}$ of $z^{\prime}$, such that the pull-back of $\left(\mathcal{C}_{\lambda} \rightarrow C \times{ }_{B} S_{\lambda} ; \mathcal{E}_{\lambda}\right)$ via $U_{z} \rightarrow S_{\lambda}$ is a Gieseker vector bundle on $C$ over $U_{z}$. Since $S$ is quasi-compact, there exist finitely many points $z_{1}, \ldots, z_{m} \in S$ such that the preimages of the $U_{z_{i}}$ by $S \rightarrow S_{\lambda_{z_{i}}}$ cover $S$. We choose an index $\alpha$ which dominates all the $\lambda_{z_{i}}$. Let $S^{\prime} \subset S_{\alpha}$ be the union of all the preimages of the $U_{z_{i}}$ by $S_{\alpha} \rightarrow S_{\lambda_{i}}$. Then $S \rightarrow S_{\alpha}$ factorizes through $S$ and the pair $\left(\mathcal{C} \rightarrow C \times{ }_{B} S^{\prime} ; \mathcal{E}\right):=\left(\mathcal{C}_{\lambda} \times{ }_{S_{\lambda}} S^{\prime} \rightarrow\right.$ $\left.C \times_{B} S^{\prime} ; \mathcal{E}_{\lambda} \otimes_{\mathcal{O}_{S_{\lambda}}} \mathcal{O}_{S^{\prime}}\right)$ is a Gieseker vector bundle with the required property.

Proposition 3.16. Let $S$ be an affine B-scheme, let $g: S^{\prime} \rightarrow S$ be an étale covering of $S$, let $S^{\prime \prime}:=S^{\prime} \times_{S} S^{\prime}$ and denote by $p_{i}: S^{\prime \prime} \rightarrow S^{\prime}$ the projection onto the $i$-th factor. Let $\left(\mathcal{C}^{\prime} \rightarrow C \times \times_{B} S^{\prime}, \mathcal{E}^{\prime}\right)$ be a Gieseker vector bundle on $C$ over $S^{\prime}$. Then every descent data

$$
f: p_{1}^{*}\left(\mathcal{C}^{\prime} \rightarrow C \times_{B} S^{\prime}, \mathcal{E}^{\prime}\right) \stackrel{\sim}{\rightarrow} p_{2}^{*}\left(\mathcal{C}^{\prime} \rightarrow C \times_{B} S^{\prime}, \mathcal{E}^{\prime}\right)
$$

for $\left(\mathcal{C}^{\prime} \rightarrow C \times_{B} S^{\prime}, \mathcal{E}^{\prime}\right)$ with respect to $S^{\prime} \rightarrow S$ is effective.

Proof. First of all we claim that it suffices to prove the proposition in the case where $S$ is a locally noetherian scheme. Indeed, by [EGA IV], (8.8.2)(ii), (8.10.5)(vi), (17.7.8)(ii), there exists an étale covering $S_{0}^{\prime} \rightarrow S_{0}$ of noetherian schemes which pulls back to the given covering via a morphism $S \rightarrow S_{0}$. Lemma 3.15 implies that we may assume that there exists a Gieseker vector bundle $\left(\mathcal{C}_{0}^{\prime} \rightarrow C \times{ }_{B} S_{0}^{\prime}, \mathcal{E}_{0}^{\prime}\right)$ which pulls back via $S^{\prime} \rightarrow S_{0}^{\prime}$ to the given one. By [EGA IV], (8.8.2)(i), (8.5.2)(i), (8.8.2.4), (8.5.2.4), we may assume that the morphism $f$ comes from a morphism $f_{0}: p_{1}^{*}\left(\mathcal{C}_{0}^{\prime} \rightarrow C \times_{B} S_{0}^{\prime}, \mathcal{E}_{0}^{\prime}\right) \stackrel{\sim}{\rightarrow} p_{2}^{*}\left(\mathcal{C}_{0}^{\prime} \rightarrow C \times_{B} S_{0}^{\prime}, \mathcal{E}_{0}^{\prime}\right)$ and that $f_{0}$ is itself a descent data. This proves our claim. So we can assume $S$ to be a locally noetherian scheme. Let $T:=C \times_{B} S, T^{\prime}: C \times_{B} S^{\prime}, T^{\prime \prime}: C \times_{B} S^{\prime \prime}$. Then $T^{\prime} \rightarrow T$ is an étale covering and $f$ induces a descent data $p_{1}^{*}\left(\mathcal{C}^{\prime}, \operatorname{det} \mathcal{E}^{\prime}\right) \stackrel{\sim}{\rightarrow} p_{2}^{*}\left(\mathcal{C}^{\prime}, \operatorname{det} \mathcal{E}^{\prime}\right)$, where $p_{i}: T^{\prime \prime}=T^{\prime} \times_{T} T^{\prime} \rightarrow T^{\prime}$ is the $i$-th projection. Since $\operatorname{det} \mathcal{E}^{\prime}$ is relatively ample with respect to the morphism $\mathcal{C}^{\prime} \rightarrow T^{\prime}$ (this follows e.g. by [EGA III], (4.7.1)), this descent data is effective ([SGA1], exp. VII, Prop 7.8). By [SGA1, exp. VII, Cor. 1.3 and Prop. 1.10, $\mathcal{E}^{\prime}$ also descends.

Proposition 3.17. Let $S$ be an affine $B$-scheme and let $\left(\mathcal{C}_{1} \rightarrow C \times \times_{B} S, \mathcal{E}_{1}\right)$, $\left(\mathcal{C}_{2} \rightarrow C \times{ }_{B} S, \mathcal{E}_{2}\right)$ be two Gieseker vector bundles on $C$ over $S$. Then the contravariant functor from the category of $S$-schemes to the category of sets, which to every $S$-scheme $T$ associates the set of isomorphisms from $\left(\mathcal{C}_{1} \rightarrow C \times{ }_{B} S, \mathcal{E}_{1}\right)_{T}$ to $\left(\mathcal{C}_{2} \rightarrow C \times_{B} S, \mathcal{E}_{2}\right)_{T}$, is representable by a quasi-compact separable $S$-scheme.

The proof of Proposition 3.17 will be given after Lemma 3.19 below.

Definition 3.18. Let $X / K$ be a projective curve over a field $K$ and let $\mathcal{L}$ be an invertible $\mathcal{O}_{X}$-module. We define $\operatorname{deg}_{X / K}(\mathcal{L}):=\chi(\mathcal{L})-\chi\left(\mathcal{O}_{X}\right)$, where for a coherent $\mathcal{O}_{X}$-module $\mathcal{F}$ we set $\chi(\mathcal{F}):=\operatorname{dim}_{K} H^{0}(X, \mathcal{F})-\operatorname{dim}_{K} H^{1}(X, \mathcal{F})$.

Lemma 3.19. Let $X$ be a prestable projective curve over an algebraically closed field and let $\mathcal{L}, \mathcal{M}$ be two invertible $\mathcal{O}_{X}$-modules. Then we have $\operatorname{deg}_{X / K}(\mathcal{L} \otimes \mathcal{M})=$ $\operatorname{deg}_{X / K}(\mathcal{L})+\operatorname{deg}_{X / K}(\mathcal{M})$.

Proof. Let $x \in X$ be an ordinary double point and let $f: \tilde{X} \rightarrow X$ be the blowing up of $X$ at $x$. Then there is an exact sequence of $\mathcal{O}_{X}$-modules as follows:

$$
0 \longrightarrow \mathcal{L} \longrightarrow f_{*} f^{*} \mathcal{L} \longrightarrow \mathcal{L}[x] \longrightarrow 0 \text {. }
$$


From this and the analogous exact sequence for $\mathcal{O}_{X}$ one deduces easily the equality $\operatorname{deg}_{X}(\mathcal{L})=\operatorname{deg}_{\tilde{X}}\left(f^{*} \mathcal{L}\right)$. This shows that we may assume $X$ to be the disjoint union of smooth projective curves or for that matter to be a single connected smooth projective curve. The Riemann-Roch theorem shows that in this case our definition of the degree of a line bundle coincides with the usual one, so the required formula holds.

Proof of Proposition 3.17, Employing techniques used in the proof of Lemma 3.15 one sees that it suffices to show that in the case of noetherian $S$ the functor $T \mapsto \operatorname{Isom}\left(\left(\mathcal{C}_{1} \rightarrow C \times_{B} S, \mathcal{E}_{1}\right)_{T},\left(\mathcal{C}_{2} \rightarrow C \times_{B} S, \mathcal{E}_{2}\right)_{T}\right)$ from the category of noetherian $S$-schemes to the category of sets is representable by a quasi-compact separable $S$-scheme. Let $\mathrm{Hilb}_{\mathcal{C}_{1} \times{ }_{S} \mathcal{C}_{2} / S}$ be the Hilbert scheme which parametrizes closed subschemes of $\mathcal{C}_{1} \times{ }_{S} \mathcal{C}_{2} / S$ which are flat over $S$. Let $Z \hookrightarrow\left(\mathcal{C}_{1} \times{ }_{S} \mathcal{C}_{2}\right) \times{ }_{S} \mathrm{Hilb}_{\mathcal{C}_{1} \times{ }_{S} \mathcal{C}_{2} / S}$ be the universal object. It is easy to see that there is a locally closed subscheme $Y \hookrightarrow \operatorname{Hilb}_{\mathcal{C}_{1} \times \mathcal{C}_{2} / S}$ with the property that a morphism $T \rightarrow \mathrm{Hilb}_{\mathcal{C}_{1} \times{ }_{S} \mathcal{C}_{2} / S}$ factorizes over $Y$ if and only if the following hold:

(1) The $i$-th projection $\operatorname{pr}_{i}: Z_{T} \rightarrow\left(\mathcal{C}_{i}\right)_{T}$ is an isomorphism for $i=1,2$.

(2) The diagram

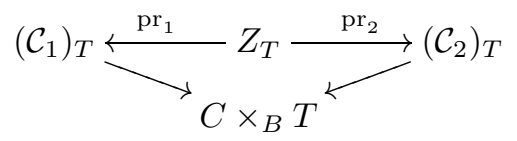

is commutative.

In particular, the pair $\left(Y, \mathrm{pr}_{2} \circ \mathrm{pr}_{1}^{-1}:\left(\mathcal{C}_{1}\right)_{Y} \stackrel{\sim}{\rightarrow} Z_{Y} \stackrel{\sim}{\rightarrow}\left(\mathcal{C}_{2}\right)_{Y}\right)$ represents the (contravariant) functor

$$
T \mapsto\left\{\text { all }\left(C \times_{B} T\right) \text {-isomorphisms }\left(\mathcal{C}_{1}\right)_{T} \stackrel{\sim}{\rightarrow}\left(\mathcal{C}_{2}\right)_{T}\right\}
$$

from $\{S$-schemes $\}$ to $\{$ sets $\}$. I claim that $Y / S$ is quasi-projective. We may assume without loss of generality that $S$ is connected. Let $\mathcal{L}_{i}$ be a very ample invertible sheaf on $\mathcal{C}_{i}$ relative to $S(i=1,2)$. Then the sheaf $\mathcal{L}:=\mathcal{L}_{1} \nabla_{\mathcal{O}_{S}} \mathcal{L}_{2}$ is very ample on $\mathcal{C}_{1} \times{ }_{X} \mathcal{C}_{2}$ over $S$. For a polynomial $P$ let $\operatorname{Hilb}_{\mathcal{C}_{1} \times{ }_{S} \mathcal{C}_{2} / S}^{P}$ be the component of Hilb $_{\mathcal{C}_{1} \times \mathcal{C}_{2} / S}$ parametrizing objects whith Hilbert polynomial $P$ with respect to the polarization $\mathcal{L}$. Since $\operatorname{Hilb}_{\mathcal{C}_{1} \times S_{S} \mathcal{C}_{2} / S}^{P}$ is projective over $S$, it is clearly enough for the proof of the claim to show that $Y$ is contained in $\operatorname{Hilb}_{\mathcal{C}_{1} \times_{S} \mathcal{C}_{2} / S}^{P}$ for some $P$. Let $T$ be the spectrum of an algebraically closed field and let $T \rightarrow S$ be a morphism. Let $Z_{T} \hookrightarrow\left(\mathcal{C}_{1} \times{ }_{S} \mathcal{C}_{2}\right)_{T}$ be a closed immersion with the above properties (1) and (2). The Hilbert polynomial $P$ of the coherent $\mathcal{O}_{\left(\mathcal{C}_{1} \times{ }_{S} \mathcal{C}_{2}\right)_{T}}$-module $\mathcal{O}_{Z_{T}}$ with respect to $\mathcal{L}_{T}$ is given by

$$
P(m)=\chi_{\left(\mathcal{C}_{1}\right)_{T}}\left(\left(\mathcal{L}_{1}\right)_{T}^{\otimes m} \otimes f_{T}^{*}\left(\mathcal{L}_{2}\right)_{T}^{\otimes m}\right),
$$

where $f_{T}:=\operatorname{pr}_{2} \circ \operatorname{pr}_{1}^{-1}:\left(\mathcal{C}_{1}\right)_{T} \stackrel{\sim}{\rightarrow}\left(\mathcal{C}_{2}\right)_{T}$. We have to show that $P$ is independent of $T \rightarrow S$ and of $Z_{T} \hookrightarrow\left(\mathcal{C}_{1} \times{ }_{S} \mathcal{C}_{2}\right)_{T}$. By Lemma 3.19 we have

$$
P(m)=\operatorname{deg}_{\left(\mathcal{C}_{1}\right)_{T}}\left(\left(\mathcal{L}_{1}\right)_{T}^{\otimes m}\right)+\operatorname{deg}_{\left(\mathcal{C}_{2}\right)_{T}}\left(\left(\mathcal{L}_{2}\right)_{T}^{\otimes m}\right)+\chi_{\left(\mathcal{C}_{1}\right)_{T}}\left(\mathcal{O}_{\left(\mathcal{C}_{1}\right)_{T}}\right)
$$

which shows the required independence. This proves our claim and in particular that $Y$ is a quasi-compact separable $S$-scheme.

Let $f:\left(\mathcal{C}_{1}\right)_{Y} \stackrel{\sim}{\rightarrow}\left(\mathcal{C}_{2}\right)_{Y}$ be the universal isomorphism. To prove the proposition it suffices now to show that the functor $T \mapsto \operatorname{Isom}_{\mathcal{O}_{\left(\mathcal{C}_{1}\right)_{T}}}\left(\left(\mathcal{E}_{1}\right)_{T}, f^{*}\left(\mathcal{E}_{2}\right)_{T}\right)$ from the 
category of $Y$-schemes to the category of sets is representable by a quasi-compact separable $Y$-scheme. But this is proven in [LM] (proof of Theorem (4.6.2.1)).

Definition 3.20. Let $C \rightarrow B$ be as in section 2, For every $B$-scheme $S$ let $H_{N}(S)$ be the set of all closed subschemes $\mathcal{C} \hookrightarrow C \times{ }_{B} \operatorname{Grass}_{n}\left(\mathcal{O}_{B}^{N}\right) \times{ }_{B} S$ with the following properties:

(1) The induced morphism $\mathcal{C} \rightarrow C \times{ }_{B} S$ is a modification of $C$ over $S$.

(2) The induced morphism $\mathcal{C} \rightarrow \operatorname{Grass}_{n}\left(\mathcal{O}_{B}^{N}\right) \times_{B} S$ is a closed immersion.

(3) Let $\mathcal{O}_{\mathcal{C}}^{N} \longrightarrow \mathcal{E}$ be the pull back by $\mathcal{C} \hookrightarrow \operatorname{Grass}_{n}\left(\mathcal{O}_{B}^{N}\right) \times{ }_{B} S$ of the universal quotient. Then for every point $z \in S$ the induced morphism $H^{0}\left(\mathcal{C}_{z}, \mathcal{O}_{\mathcal{C}_{z}}^{N}\right) \rightarrow$ $H^{0}\left(\mathcal{C}_{z}, \mathcal{E}_{z}\right)$ is an isomorphism and the cohomology groups $H^{i}\left(\mathcal{C}_{z}, \mathcal{E}_{z}\right)$ vanish for $i \geq 1$.

(4) The $\mathcal{O}_{\mathcal{C}}$-module $\mathcal{E}$ is admissible for $\mathcal{C} \rightarrow C \times_{B} S$.

The association $S \mapsto H_{N}(S)$ is in an obvious way a contravariant functor $H_{N}$ from the category of $B$-schemes to the category of sets.

Theorem 3.21 ( $[\mathrm{NS}]$ ). Assume that the residue field $k$ of $B$ is algebraically closed and of characteristic zero. Then the functor $H_{N}$ defined in 3.20 is represented by a subscheme (also denoted $H_{N}$ ) of the Hilbert scheme $\operatorname{Hilb}_{C \times{ }_{B} \operatorname{Grass}_{n}\left(\mathcal{O}_{B}^{N}\right) / B}$. Furthermore, the scheme $H_{N}$ is regular and flat over $B$, its generic fibre (over $B$ ) is smooth and its special fibre is a divisor with normal crossings in $H_{N}$.

Proof. By [NS], Prop. 8, there exists an open subscheme $\mathcal{Y}$ of the Hilbert scheme $\operatorname{Hilb}_{C \times{ }_{B} \operatorname{Grass}_{n}\left(\mathcal{O}_{B}^{N}\right) / B}$ which parametrizes closed subschemes satisfying properties (1)-(3) in Definition 3.20, Furthermore, $\mathcal{Y}$ is regular, its generic fibre over $B$ is smooth and its special fibre is a divisor with normal crossings. Let $S$ be a $B$ scheme and let $\mathcal{C} \hookrightarrow C \times_{B} \operatorname{Grass}_{n}\left(\mathcal{O}_{B}^{N}\right) \times_{B} S$ be an $S$-valued point of $\mathcal{Y}$. Let $h: \mathcal{C} \rightarrow C \times_{B} S$ be the projection and let $\mathcal{O}_{\mathcal{C}}^{N} \longrightarrow \mathcal{E}$ be the pull-back by $\mathcal{C} \hookrightarrow \operatorname{Grass}_{n}\left(\mathcal{O}_{B}^{N}\right) \times_{B} S$ of the universal quotient. In [NS], Prop. 9, it is shown that application of $h_{*}$ gives a surjection $\mathcal{O}_{C \times_{B} S}^{N} \longrightarrow h_{*} \mathcal{E}$ such that $h_{*} \mathcal{E}$ is flat over $S$. In particular, push-forward induces a morphism from $\mathcal{Y}$ to the Quot-scheme Quot $_{\mathcal{O}_{\mathcal{C}}^{N} / \mathcal{C} / B}$ which parametrizes coherent quotients of $\mathcal{O}_{C}^{N}$ which are flat over the base. Let $\mathcal{Q} \subset \operatorname{Quot}_{\mathcal{O}_{\mathcal{C}}^{N} / \mathcal{C} / B}$ be the open subscheme parametrizing torsion-free quotients. I claim that the preimage of $\mathcal{Q}$ by the morphism $\mathcal{Y} \rightarrow$ Quot $_{\mathcal{O}_{\mathcal{C}}^{N} / \mathcal{C} / B}$ represents the functor $H_{N}$. Indeed, let $S$ be a $B$-scheme which is the spectrum of a field, let $\mathcal{C} \hookrightarrow C \times{ }_{B} \operatorname{Grass}_{n}\left(\mathcal{O}_{B}^{N}\right) \times_{B} S$ be an $S$-valued point of $\mathcal{Y}$, let $h: \mathcal{C} \rightarrow C \times{ }_{B} S$ be the projection and let $\mathcal{E}$ be the pull-back of the universal quotient. Property (2) in Definition 3.20 ensures that $\mathcal{E}$ is strictly positive. Therefore Lemma 3.7 tells us that $\mathcal{E}$ is admissible for $h$ if and only if $h_{*} \mathcal{E}$ is torsion-free.

Definition 3.22. We fix a very ample invertible sheaf $\mathcal{O}_{C}(1)$ on the curve $C$. For a Gieseker vector bundle $\left(f: \mathcal{C} \rightarrow C \times_{B} S, \mathcal{E}\right)$ on $C$ over a $B$-scheme $S$ and for an integer $N^{\prime}$ we denote by $\mathcal{E}\left(-N^{\prime}\right)$ the (admissible) $\mathcal{O}_{\mathcal{C}}$-module $\mathcal{E} \otimes_{\mathcal{O}_{\mathcal{C}}}$ $f^{*}\left(\mathcal{O}_{C}(1)^{\otimes\left(-N^{\prime}\right)} \bigotimes_{\mathcal{O}_{B}} \mathcal{O}_{S}\right)$. For every pair of integers $N \geq n, N^{\prime} \geq 0$ we have a morphism of $B$-groupoids

$$
P_{N, N^{\prime}}: H_{N} \rightarrow \mathrm{GVB}_{n}(C / B)
$$


which to an $S$-valued point $\left(\mathcal{C} \hookrightarrow C \times_{B} \times \operatorname{Grass}_{n}\left(\mathcal{O}_{B}^{N}\right) \times_{B} S\right)$ of $H_{N}$ associates the $S$-valued point $\left(\mathcal{C} \rightarrow C \times \times_{B} S, \mathcal{E}\left(-N^{\prime}\right)\right)$ of $\operatorname{GVB}_{n}(C / B)$, where $\mathcal{E}$ is the pull back by $\mathcal{C} \hookrightarrow \operatorname{Grass}_{n}\left(\mathcal{O}_{B}^{N}\right) \times{ }_{B} S$ of the universal quotient sheaf on the Grassmannian.

Lemma 3.23. Let $S$ be an affine B-scheme and let $\left(\mathcal{C} \rightarrow C \times_{B} S, \mathcal{E}\right)$ be Gieseker vector bundle of rank $n$ on $C$ over $S$. Let $N \geq n$ and $N^{\prime} \geq 0$. Then there is an open subscheme $S_{N, N^{\prime}} \subseteq S$ such that a morphism $T \rightarrow S$ factorizes over $S_{N, N^{\prime}}$ if and only if for every point $t \in T$ the following hold:

(1) $\operatorname{dim} H^{i}\left(\mathcal{C}_{t}, \mathcal{E}\left(N^{\prime}\right)_{t}\right)= \begin{cases}N & \text { for } i=0, \\ 0 & \text { else. }\end{cases}$

(2) The canonical morphism $H^{0}\left(\mathcal{C}_{t}, \mathcal{E}\left(N^{\prime}\right)_{t}\right) \otimes_{\kappa(t)} \mathcal{O}_{\mathcal{C}_{t}} \longrightarrow \mathcal{E}\left(N^{\prime}\right)_{t}$ is surjective.

(3) The morphism $\mathcal{C}_{t} \rightarrow \operatorname{Grass}_{n}\left(H^{0}\left(\mathcal{C}_{t}, \mathcal{E}\left(N^{\prime}\right)_{t}\right)\right)$ induced by the surjection in (2) is a closed immersion.

Proof. By 3.15 one is easily reduced to the case where $S$ is noetherian. We define subsets $S^{\prime \prime \prime} \subseteq S^{\prime \prime} \subseteq S^{\prime} \subseteq S$ as follows: $S^{\prime}$ is the set of points $s \in S$ with the property that $H^{i}\left(\mathcal{C}_{s}, \mathcal{E}\left(N^{\prime}\right)_{s}\right)$ vanishes if $i>0$ and is of dimension $N$ if $i=0$. $S^{\prime \prime}$ is the set of points in $s \in S^{\prime}$ such that the canonical morphism $H^{0}\left(\mathcal{C}_{s}, \mathcal{E}\left(N^{\prime}\right)_{s}\right) \otimes_{\kappa(s)}$ $\mathcal{O}_{\mathcal{C}_{s}} \rightarrow \mathcal{E}\left(N^{\prime}\right)_{s}$ is surjective. Finally, $S^{\prime \prime \prime}$ is the set of points $s \in S^{\prime \prime}$ with the property that the morphism $\mathcal{C}_{s} \rightarrow \operatorname{Grass}_{n}\left(H^{0}\left(\mathcal{C}_{s}, \mathcal{E}\left(N^{\prime}\right)\right)\right)$ induced by the surjection $H^{0}\left(\mathcal{C}_{s}, \mathcal{E}\left(N^{\prime}\right)_{s}\right) \otimes_{\kappa(s)} \mathcal{O}_{\mathcal{C}_{s}} \rightarrow \mathcal{E}\left(N^{\prime}\right)_{s}$ is a closed immersion. I claim that $S^{\prime \prime \prime}$ is open in $S$. Indeed $S^{\prime}$ is open in $S$ by the semicontinuity theorem (cf. [EGA III], (7.7.5.1)) and by EGA III, (7.9.9). Since $\mathcal{C} \rightarrow S$ is proper, an application of Nakayama's lemma shows that $S^{\prime \prime}$ is open in $S^{\prime}$. The openness of $S^{\prime \prime \prime}$ follows from Lemma 3.13 It is clear that $S_{N, N^{\prime}}:=S^{\prime \prime \prime}$ has all the required properties.

Proposition 3.24. The morphism of B-groupoids

$$
P:=\coprod_{\substack{N \geq n \\ N N^{\prime} \geq 0}} P_{N, N^{\prime}}: \coprod_{\substack{N \geq n \\ N^{\prime} \geq 0}} H_{N} \longrightarrow G V B_{n}(C / B)
$$

is smooth and surjective.

Proof. Let $S$ be an affine $B$-scheme and let $S \rightarrow \operatorname{GVB}(C / B)$ be an $S$-valued point of $\operatorname{GVB}(C / B)$, given by a Gieseker vector bundle $\left(\mathcal{C} \rightarrow C \times{ }_{B} S, \mathcal{E}\right)$ on $C$ over $S$. Let $Y$ be the $B$-groupoid defined by the cartesian diagram

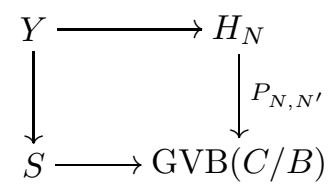

I claim that $Y=\operatorname{Isom}\left(\mathcal{O}_{S_{N, N^{\prime}}}^{N},\left.\pi_{*} \mathcal{E}\left(N^{\prime}\right)\right|_{S_{N, N^{\prime}}}\right)$, where $\pi: \mathcal{C} \rightarrow S$ is the structure morphism and where $S_{N, N^{\prime}} \subseteq S$ is the open subscheme defined with respect to $\left(\mathcal{C} \rightarrow C \times{ }_{B} S, \mathcal{E}\right)$ in Lemma 3.23 .

Let $U$ be an $S$-scheme. By definition, a $U$-valued point of $Y$ is given by the following data:

(1) A closed subscheme $\mathcal{C}^{\prime} \hookrightarrow C \times \times_{B} \operatorname{Grass}_{n}\left(\mathcal{O}_{B}^{N}\right) \times_{B} U$ with the properties (1)-(4) listed in Definition 3.20.

(2) An isomorphism $\varphi:\left.\mathcal{C}\right|_{U} \stackrel{\sim}{\rightarrow} \mathcal{C}^{\prime}$ of $U$-schemes. 
(3) An isomorphism $\left.\mathcal{E}\left(N^{\prime}\right)\right|_{U} \stackrel{\sim}{\rightarrow} \varphi^{*} \mathcal{E}^{\prime}$, where $\mathcal{O}_{\mathcal{C}^{\prime}}^{N} \longrightarrow \mathcal{E}^{\prime}$ is the pull-back of the canonical quotient on the Grassmannian.

Observe that $U \rightarrow S$ factorizes over $S_{N, N^{\prime}}$. Indeed this follows easily from the characterization of $S_{N, N^{\prime}}$ given in Lemma 3.23 and the fact (cf. 3.9) that for any $z \in S$ we have $H^{0}\left(\mathcal{C}_{z}, \mathcal{O}_{\mathcal{C}_{z}}\right)=\kappa(z)$. By 3.9 and 3.20 (3) we have $\mathcal{O}_{U}^{N}=\left.\pi_{*}^{\prime} \mathcal{O}_{\mathcal{C}^{\prime}}^{N}\right|_{U} \stackrel{\sim}{\rightarrow}$ $\left.\pi_{*}^{\prime} \mathcal{E}^{\prime}\right|_{U}$. Therefore the above data induce an isomorphism $\left.\mathcal{O}_{U}^{N} \stackrel{\sim}{\rightarrow} \pi_{*} \mathcal{E}\left(N^{\prime}\right)\right|_{U}$, i.e. a $U$-valued point of $\operatorname{Isom}\left(\mathcal{O}_{S_{N, N^{\prime}}}^{N},\left.\pi_{*} \mathcal{E}\left(N^{\prime}\right)\right|_{S_{N, N^{\prime}}}\right)$.

Conversely, let $U$ be an $S_{N, N^{\prime}}$-scheme and let $\left.\mathcal{O}_{U}^{N} \stackrel{\sim}{\rightarrow} \pi_{*} \mathcal{E}\left(N^{\prime}\right)\right|_{U}$ be a $U$-valued point of $\operatorname{Isom}\left(\mathcal{O}_{S_{N, N^{\prime}}}^{N},\left.\pi_{*} \mathcal{E}\left(N^{\prime}\right)\right|_{S_{N, N^{\prime}}}\right)$. Then we have:

(1) Let $\left(\mathcal{C}_{U} \rightarrow C \times \times_{B} U, \mathcal{E}_{U}\right)$ be the pull back of $\left(\mathcal{C} \rightarrow C \times{ }_{B} S, \mathcal{E}\right)$ to $U$ and let $\pi_{U}: \mathcal{C}_{U} \rightarrow U$ be the projection. Then we have a canonical morphism $\mathcal{O}_{\mathcal{C}_{U}}^{N} \stackrel{\sim}{\rightarrow} \pi_{U}^{*}\left(\pi_{U}\right)_{*} \mathcal{E}_{U}\left(N^{\prime}\right) \rightarrow \mathcal{E}_{U}\left(N^{\prime}\right)$, which by definition of $S_{N, N^{\prime}}$ (cf. Lemma 3.23) is surjective and induces a closed immersion $\mathcal{C}_{U} \hookrightarrow \operatorname{Grass}_{n}\left(\mathcal{O}_{B}^{N}\right) \times_{B} U$. Thus we have a closed immersion $\mathcal{C}_{U} \hookrightarrow$ $C \times{ }_{B} \operatorname{Grass}_{n}\left(\mathcal{O}_{B}^{N}\right) \times_{B} U$ which obviously has the properties (1)-(4) listed in 3.20 .

(2) The identity isomorphism $\left.\mathcal{C}\right|_{U} \stackrel{\sim}{\rightarrow} \mathcal{C}_{U}$.

(3) The natural isomorphism $\left.\mathcal{E}\left(N^{\prime}\right)\right|_{U}=\mathcal{E}_{U}\left(N^{\prime}\right) \stackrel{\sim}{\rightarrow} \mathcal{E}^{\prime}$, where $\mathcal{O}_{\mathcal{C}_{U}}^{N} \rightarrow \mathcal{E}^{\prime}$ is the pull back of the canonical quotient of the Grassmannian.

These are the data needed to define a morphism $U \rightarrow Y$. Thus we have established a correspondence between $U$-valued points of $Y$ and of $\operatorname{Isom}\left(\mathcal{O}_{S_{N, N^{\prime}}}^{N},\left.\pi_{*} \mathcal{E}\left(N^{\prime}\right)\right|_{S_{N, N^{\prime}}}\right)$ which is easily seen to be a bijection functorial in $U$. This proves our claim.

Since $\operatorname{Isom}\left(\mathcal{O}_{S_{N, N^{\prime}}}^{N},\left.\pi_{*} \mathcal{E}\left(N^{\prime}\right)\right|_{S_{N, N^{\prime}}}\right)$ is smooth and surjective over $S_{N, N^{\prime}}$, all that remains to be shown is that the open subschemes $\left(S_{N, N^{\prime}}\right)_{N \geq n, N^{\prime} \geq 0}$ cover $S$. But this is an immediate consequence of [NS], Proposition 4.

\section{Gieseker VECTOR BUNDle DATA}

Definition 4.1. Let $K$ be a field, $\tilde{X} / K$ a curve and $x^{\prime}, x^{\prime \prime}$ two different smooth $K$-rational points of $\tilde{X}$. A modification of $\left(\tilde{X}, x^{\prime}, x^{\prime \prime}\right)$ is a morphism $\left(Y, y^{\prime}, y^{\prime \prime}\right) \rightarrow$ $\left(\tilde{X}, x^{\prime}, x^{\prime \prime}\right)$ of two-pointed curves, such that

(1) $Y$ is isomorphic to the cokernel of the double arrow (cf. section 2)

$$
\operatorname{Spec}(K) \amalg \operatorname{Spec}(K) \underset{x_{0}^{\prime} \amalg x_{0}^{\prime \prime}}{\stackrel{x^{\prime} \amalg x^{\prime \prime}}{\rightleftarrows}} \tilde{X} \amalg R^{\prime} \amalg R^{\prime \prime}
$$

where $R^{\prime}$ (resp. $R^{\prime \prime}$ ) is a chain of rational curves over $K$ of length $r^{\prime}$ (resp. $\left.r^{\prime \prime}\right)$ and $x_{0}^{\prime}, x_{r^{\prime}}^{\prime}\left(\right.$ resp. $\left.x_{0}^{\prime \prime}, x_{r^{\prime \prime}}^{\prime \prime}\right)$ are smooth $K$ rational points of $R^{\prime}$ (resp. of $\left.R^{\prime \prime}\right)$, which lie on different extremal components if $r^{\prime} \geq 2$ (resp. if $r^{\prime \prime} \geq 2$ ), are not equal if $r^{\prime}=1$ (resp. if $r^{\prime \prime}=1$ ), and are identical if $r^{\prime}=0$ (resp. if $\left.r^{\prime \prime}=0\right)$.

(2) The composed morphism $\tilde{X} \rightarrow Y \rightarrow \tilde{X}$ is the identity, the composed morphism $R^{\prime} \rightarrow Y \rightarrow \tilde{X}$ (resp. $R^{\prime \prime} \rightarrow Y \rightarrow \tilde{X}$ ) is the constant morphism onto $x^{\prime}$ (resp. onto $\left.x^{\prime \prime}\right)$ and $y^{\prime}$ (resp. $\left.y^{\prime \prime}\right)$ is the $K$-rational point induced by $x_{r^{\prime}}^{\prime}$ (resp. by $\left.x_{r^{\prime \prime}}^{\prime \prime}\right)$. 
The situation may be visualized as follows:

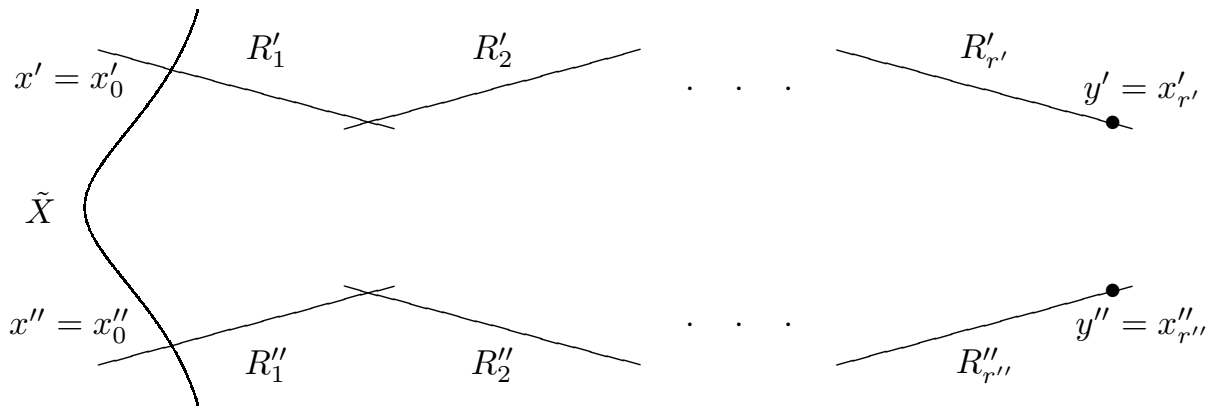

Definition 4.2. Let $h:\left(Y, y^{\prime}, y^{\prime \prime}\right) \rightarrow\left(\tilde{X}, x^{\prime}, x^{\prime \prime}\right)$ be a modification of $\left(\tilde{X}, x^{\prime}, x^{\prime \prime}\right)$ as in Definition 4.1. A locally free $\mathcal{O}_{Y^{\prime}}$-module $\mathcal{E}$ is called admissible for $\left(Y, y^{\prime}, y^{\prime \prime}\right) \rightarrow$ $\left(\tilde{X}, x^{\prime}, x^{\prime \prime}\right)$ if the restrictions of $\mathcal{E}$ to the two chains of rational curves $R^{\prime}=h^{-1}\left(x^{\prime}\right)$ and $R^{\prime \prime}=h^{-1}\left(x^{\prime \prime}\right)$ are both admissible in the sense of 3.2. The extremal degree of an admissible $\mathcal{O}_{Y}$-module $\mathcal{E}$ of rank $n$ is the pair of numbers $\left(d^{\prime}, d^{\prime \prime}\right) \in[1, n+1]^{2}$, where for $\iota \in\left\{{ }^{\prime},{ }^{\prime \prime}\right\}$ the number $d^{\iota}$ is the degree of $\left.\mathcal{E}\right|_{R_{r \iota}^{\iota}}$ if $R^{\iota}$ is of length $r^{\iota} \geq 1$, where $R_{r^{\iota}}^{\iota}$ is the component of $R^{\iota}$ which contains the point $y^{\iota}$, and $d^{\iota}:=\infty$ if $R^{\iota}$ is of length 0 . A pair consisting of a locally free $\mathcal{O}_{Y}$-module $\mathcal{E}$ and an isomorphism $\varphi: \mathcal{E}\left[y^{\prime}\right] \stackrel{\sim}{\rightarrow} \mathcal{E}\left[y^{\prime \prime}\right]$ is called admissible for $\left(Y, y^{\prime}, y^{\prime \prime}\right) \rightarrow\left(\tilde{X}, x^{\prime}, x^{\prime \prime}\right)$ if the the module induced by $(\mathcal{E}, \varphi)$ on the chain $R:=\left(R^{\prime} \amalg R^{\prime \prime}\right) /\left(y^{\prime}=y^{\prime \prime}\right)$ of rational curves of length $r^{\prime}+r^{\prime \prime}$ (by identifying via $\varphi$ the fibres at the points $y^{\prime}$ and $y^{\prime \prime}$ ) is admissible in the sense of Definition 3.2

Remark 4.3. With the notation in the above definition, admissibility of the pair $(\mathcal{E}, \varphi)$ implies admissibility of $\mathcal{E}$ for $\left(Y, y^{\prime}, y^{\prime \prime}\right) \rightarrow\left(\tilde{X}, x^{\prime}, x^{\prime \prime}\right)$. Cf. the end of Remark 3.4 .

Definition 4.4. Let $C_{0} \rightarrow \operatorname{Spec}(k)$ be the curve from section 2 and let $\left(\tilde{C}_{0}, p_{1}, p_{2}\right)$ be the normalization together with its two distinguished points. Let $S$ be a $k$ scheme. A modification of $\left(\tilde{C}_{0}, p_{1}, p_{2}\right)$ over $S$ is a diagram

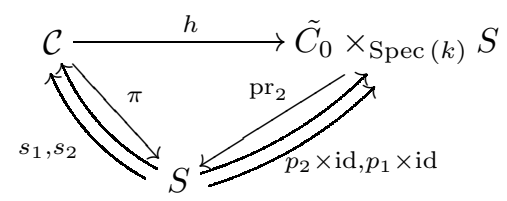

which is commutative in the sense that $\operatorname{pr}_{2} \circ h=\pi$ and $h \circ s_{i}=p_{i} \times$ id for $i=1,2$, where $\pi$ is flat, $h$ is proper and finitely presented, the $s_{i}$ are sections of $\pi$, and for every point $z \in S$ the induced morphism on the fibre $\left(\mathcal{C}_{z}, s_{1}(z), s_{2}(z)\right) \rightarrow$ $\left(\tilde{C_{0}}, p_{1}, p_{2}\right) \times_{\operatorname{Spec}(k)} \operatorname{Spec}(\kappa(z))$ is a modification of $\left(\tilde{C}_{0}, p_{1}, p_{2}\right) \times_{\operatorname{Spec}(k)} \operatorname{Spec}(\kappa(z))$ in the sense of Definition 4.1 .

Definition 4.5. Let $\left(\mathcal{C}, \pi, s_{1}, s_{2}, h\right)$ be a modification of $\left(\tilde{C}_{0}, p_{1}, p_{2}\right)$ over $S$ as in Definition 4.4. A locally free $\mathcal{O}_{\mathcal{C}}$-module $\mathcal{E}$ is called admissible for $\left(\mathcal{C}, \pi, s_{1}, s_{2}, h\right)$ if for all $z \in S$ the restriction of $\mathcal{E}$ to the fibre $\mathcal{C}_{z}$ is admissible for $\left(\mathcal{C}_{z}, s_{1}(z), s_{2}(z)\right) \rightarrow$ $\left(\tilde{C}_{0}, p_{1}, p_{2}\right) \times_{S p e c(k)} \operatorname{Spec}(\kappa(z))$ in the sense of Definition 4.2. We define $\mathcal{E}$ to be of extremal degree $\geq\left(d_{1}, d_{2}\right)$ if for all $z \in S$ we have $d_{z}^{\prime} \geq d_{1}$ and $d_{z}^{\prime \prime} \geq d_{2}$, where $\left(d_{z}^{\prime}, d_{z}^{\prime \prime}\right)$ is the extremal degree of $\left.\mathcal{E}\right|_{\mathcal{C}_{z}}$ in the sense of 4.2. A pair consisting of a 
locally free $\mathcal{O}_{\mathcal{C}}$-module $\mathcal{E}$ and an isomorphism $\varphi: s_{1}^{*} \mathcal{E} \stackrel{\sim}{\rightarrow} s_{2}^{*} \mathcal{E}$ is called admissible for $\left(\mathcal{C}, \pi, s_{1}, s_{2}, h\right)$ if it is fibrewise admissible in the sense of 4.2 .

Lemma 4.6. Let $\left(\tilde{C}_{0}, p_{1}, p_{2}\right)$ be the two-pointed $k$-curve from section 2 and let $S^{\prime}$ be an affine $k$-scheme. Let $\left(\mathcal{C}^{\prime}, \pi^{\prime}, s_{1}^{\prime}, s_{2}^{\prime}, h^{\prime}\right)$ be a modification of $\left(\tilde{C}_{0}, p_{1}, p_{2}\right)$ over $S^{\prime}$ and let $\mathcal{E}^{\prime}$ be a locally free $\mathcal{O}_{\mathcal{C}^{\prime}}$-module which is admissible for $\left(\mathcal{C}^{\prime}, \pi^{\prime}, s_{1}^{\prime}, s_{2}^{\prime}, h^{\prime}\right)$. Then there exists a $k$-scheme of finite type $S$, a modification $\left(\mathcal{C}, \pi, s_{1}, s_{2}, h\right)$ of $\left(\tilde{C}_{0}, p_{1}, p_{2}\right)$ over $S$, a locally free $\mathcal{O}_{\mathcal{C}}$-module admissible for $\left(\mathcal{C}, \pi, s_{1}, s_{2}, h\right)$ and a $k$ morphism $S^{\prime} \rightarrow S$ such that $\left(\mathcal{C}^{\prime}, \pi^{\prime}, s_{1}^{\prime}, s_{2}^{\prime}, h^{\prime} ; \mathcal{E}^{\prime}\right)$ is induced by pull-back via $S^{\prime} \rightarrow S$ from $\left(\mathcal{C}, \pi, s_{1}, s_{2}, h ; \mathcal{E}\right)$.

Proof. We omit the proof which is similar to the proof of Lemma 3.15

Definition 4.7. Let $\left(\tilde{C}_{0}, p_{1}, p_{2}\right)$ be the two-pointed $k$-curve from section 2 and let $S$ be a $k$-scheme. A Gieseker vector bundle data (of rank $n$ ) on $\left(\tilde{C}_{0}, p_{1}, p_{2}\right)$ over $S$ is a modification $\left(\mathcal{C}, \pi, s_{1}, s_{2}, h\right)$ of $\left(\tilde{C}_{0}, p_{1}, p_{2}\right)$ over $S$ together with a locally free $\mathcal{O}_{\mathcal{C}}$-module $\mathcal{E}$ (of rank $n$ ) and an isomorphism $\varphi: s_{1}^{*} \mathcal{E} \stackrel{\sim}{\rightarrow} s_{2}^{*} \mathcal{E}$, such that $(\mathcal{E}, \varphi$ ) is admissible for $\left(\mathcal{C}, \pi, s_{1}, s_{2}, h\right)$.

As all Gieseker vector bundle data of rank $n$ on $\left(C_{0}, p_{1}, p_{2}\right)$ over a fixed $k$-scheme $S$ form in an obvious way a groupoid, and as there is a natural notion of pull-back under a morphism $S^{\prime} \rightarrow S$ of such data, we have the following functor:

$\operatorname{GVBD}_{n}\left(\tilde{C}_{0}, p_{1}, p_{2}\right):\left\{\begin{array}{clc}\{k \text {-schemes }\}^{\circ} & \rightarrow & \text { \{groupoids }\} \\ S & \mapsto\left(\begin{array}{c}\text { Gieseker vector bundle data } \\ \text { of rank } n \text { on }\left(C_{0}, p_{1}, p_{2}\right) \text { over } S\end{array}\right)\end{array}\right.$

Again, we often suppress the index $n$ in the notation of this $k$-groupoid.

Let $S$ be a $k$ scheme and let $\left(\tilde{\mathcal{C}}, \tilde{\pi}, s_{1}, s_{2}, \tilde{h}, \tilde{\mathcal{E}}, \varphi\right)$ be an object in

$$
\operatorname{GVBD}\left(\tilde{C}_{0}, p_{1}, p_{2}\right)(S)
$$

Let $\mathcal{C}$ be the cokernel of the double arrow (cf. section 2)

$$
S \stackrel{s_{1}}{\underset{s_{2}}{\longrightarrow}} \tilde{C}
$$

and let $\pi: \mathcal{C} \rightarrow S$ the morphism induced by $\tilde{\pi}$. By the universal property of $\mathcal{C}$ (cf. Kn, Theorem 3.4), $\tilde{h}$ induces an $S$-morphism $h: \mathcal{C} \rightarrow C_{0} \times_{\text {Spec (k) }} S$. Furthermore, the data $(\tilde{\mathcal{E}}, \varphi)$ induces a bundle $\mathcal{E}$ on $\mathcal{C}$ which clearly is admissible for $h$. Thus we have constructed an object $(\mathcal{C}, \pi, h, \mathcal{E})$ in $\operatorname{GVB}\left(C_{0} / k\right)(S)$. Since the construction

$$
\left(\tilde{\mathcal{C}}, \tilde{\pi}, s_{1}, s_{2}, \tilde{h}, \tilde{\mathcal{E}}, \varphi\right) \mapsto(\mathcal{C}, \pi, h, \mathcal{E})
$$

is functorial with respect to isomorphisms and commutes with base-change by a morphism $S^{\prime} \rightarrow S$, we obtain a 1-morphism $\operatorname{GVBD}\left(\tilde{C}_{0}, p_{1}, p_{2}\right) \rightarrow \operatorname{GVB}\left(C_{0} / k\right)$ of $k$-groupoids.

Definition 4.8 (cf. [D]). Let $S$ be a scheme and let $\mathcal{Y}$ be a reduced algebraic $S$-stack. An algebraic $S$-stack $\mathcal{X}$ together with a representable 1-morphism $\mathcal{X} \rightarrow \mathcal{Y}$ is called the normalization of $\mathcal{Y}$ if there exists a cartesian diagram

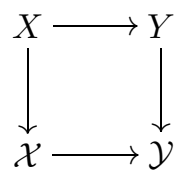


where $X$ and $Y$ are algebraic spaces, $Y \rightarrow \mathcal{Y}$ is a smooth surjective 1-morphism and $X$ is the normalization of $Y$.

Theorem 4.9. Assume that $k$ is algebraically closed and of characteristic zero. Then the natural morphism of $k$-groupoids

$$
\operatorname{GVBD}\left(\tilde{C}_{0}, p_{1}, p_{2}\right) \rightarrow G V B\left(C_{0} / k\right)
$$

identifies $\operatorname{GVBD}\left(\tilde{C}_{0}, p_{1}, p_{2}\right)$ with the normalization of $G V B\left(C_{0} / k\right)$.

The proof of the theorem will be given after 4.11 below.

Lemma 4.10. Let $k$ be an algebraically closed field and let $X^{\prime} \rightarrow X$ be a proper morphism of $k$-schemes which are of finite type over $k$. Assume that for every closed point $x \in X$ there exist numbers $1 \leq m \leq n$ and a commutative diagram

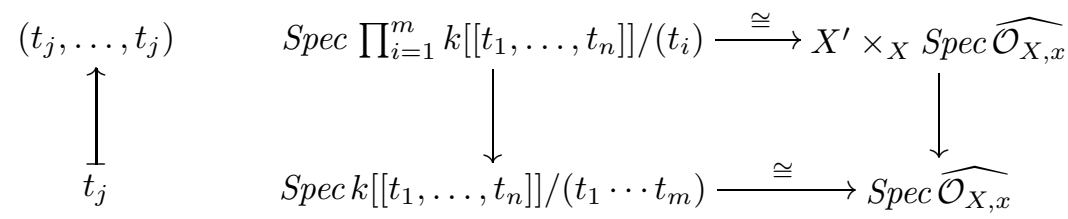

where the horizontal arrows are isomorphisms and the left vertical arrow is defined as indicated. Assume furthermore that $X^{\prime} \rightarrow X$ is an isomorphism outside the singular locus of $X$. Then $X$ is reduced, $X^{\prime}$ is smooth and $X^{\prime} \rightarrow X$ is the normalization of $X$.

Proof. The assumptions clearly imply that $X^{\prime}$ is smooth and that $X$ is reduced. Let $\left(X_{i}^{\prime}\right)_{i \in I}$ and $\left(X_{j}\right)_{j \in J}$ be the irreducible components of $X^{\prime}$ and $X$ respectively. Since each $X_{j}$ is generically smooth and by assumption $X^{\prime} \rightarrow X$ is an isomorphism outside the singular locus of $X$, it follows that we may identify the index sets $I$ and $J$ such that $X^{\prime} \rightarrow X$ induces a birational morphism $X_{i}^{\prime} \rightarrow X_{i}$ for all $i \in I$. By [EGA II], (6.3.9), the morphism $X_{i}^{\prime} \rightarrow X_{i}$ factorizes uniquely over the normalization $\tilde{X}_{i} \rightarrow X_{i}$ of $X_{i}$. The induced morphism $X_{i}^{\prime} \rightarrow \tilde{X}_{i}$ is proper, birational and quasifinite and hence an isomorphism by [EGA III], (4.4.9). Therefore $X^{\prime}=\amalg_{i} X_{i}^{\prime} \stackrel{\sim}{\rightarrow} \amalg_{i} \tilde{X}_{i}=\tilde{X}$ is the normalization of $X$ (cf. [EGA II], (6.3.8)).

Construction 4.11. Let $r \geq 0$. For $l \in[0, r]$ let

$$
V_{l}:=\operatorname{Spec}\left(k\left[\left[t_{0}, \ldots, t_{l-1}, t_{l+1}, \ldots, t_{r}\right]\right]\right) .
$$

We define modifications $\left(\tilde{Z}_{l}^{(i)}, \pi^{(i)}, s_{1}^{(i)}, s_{2}^{(i)}, h^{(i)}\right)$ of $\left(\tilde{C}_{0}, p_{1}, p_{2}\right)$ over $V_{l}$ for $i=$ $0, \ldots, l-1$ inductively as follows. Let $\tilde{Z}_{l}^{(0)}:=\tilde{C}_{0} \times_{k} V_{l}$, let the sections $s_{\nu}^{(0)}$ be induced by the points $p_{\nu}$, let the morphism $\pi^{(0)}$ be the projection onto $V_{l}$ and let $h^{(0)}$ be the identity morphism. Assume that $\left(\tilde{Z}_{l}^{(i)}, \pi^{(i)}, s_{1}^{(i)}, s_{2}^{(i)}, h^{(i)}\right)$ has already been constructed for some $i \in[0, l-2]$. Then $\tilde{Z}_{l}^{(i+1)}$ is defined to be the blowing up of $\tilde{Z}_{l}^{(i)}$ along the closed subscheme $s_{1}^{(i)}\left(\left\{t_{i}=0\right\}\right)$. The morphisms $\pi^{(i+1)}$ and $h^{(i+1)}$ are by definition the composition of the morphism $\tilde{Z}_{l}^{(i+1)} \rightarrow \tilde{Z}_{l}^{(i)}$ with the morphisms $\pi^{(i)}$ and $h(i)$ respectively, and the sections $s_{\nu}^{(i+1)}$ are the proper transforms of the sections $s_{\nu}^{(i)}$. Now let $\left(\tilde{Z}_{l}^{[0]}, \pi^{[0]}, s_{1}^{[0]}, s_{2}^{[0]}, h^{[0]}\right):=\left(\tilde{Z}_{l}^{(l-1)}, \pi^{(l-1)}, s_{1}^{(l-1)}, s_{2}^{(l-1)}, h^{(l-1)}\right)$ and define $\left(\tilde{Z}_{l}^{[i]}, \pi^{[i]}, s_{1}^{[i]}, s_{2}^{[i]}, h^{[i]}\right)$ for $i:=1, \ldots, r-l$ inductively as follows: For $i \geq 0$ the scheme $\tilde{Z}_{l}^{[i+1]}$ is the blowing up of the scheme $\tilde{Z}_{l}^{[i]}$ along the closed subscheme $s_{2}^{[i]}\left(\left\{t_{r-i}=0\right\}\right)$. The morphisms $\pi^{[i+1]}$ and $h^{[i+1]}$ are again the composition 
of the blowing-up morphism with the morphisms $\pi^{[i]}$ and $h^{[i]}$ respectively and the sections $s_{\nu}^{[i+1]}$ are the proper transforms of the sections $s_{\nu}^{[i]}$. Let $Z_{l}$ be the cokernel of the double arrow (cf. section 2)

$$
V_{l} \stackrel{s_{1}}{\longrightarrow} \tilde{Z}_{l}^{[r-l]}
$$

where $s_{\nu}:=s_{\nu}^{[r-l]}$. Then the morphism $h^{[r-l]}$ factorizes over $Z_{l}$ and the induced morphism $Z_{l} \rightarrow V_{l}$ is a modification of $C_{0}$ over $V_{l}$. Furthermore, the sections $s_{1}$ and $s_{2}$ induce a section $\sigma_{l}$ of $Z_{l} \rightarrow V_{l}$. Observe that the special fibre of $Z_{l} \rightarrow V_{l}$ contains a chain of projective lines of length $r$ and that $\sigma_{l}$ cuts this chain into two chains of length $l$ and $r-l$ respectively.

Let $V:=\operatorname{Spec}\left(k\left[\left[t_{0}, \ldots, t_{r}\right]\right] /\left(t_{1} \ldots t_{r}\right)\right)$ and let $V_{l} \hookrightarrow V$ be the closed immersion defined by

$$
\begin{aligned}
\left.k\left[\left[t_{0}, \ldots, t_{r}\right]\right] /\left(t_{1} \ldots t_{r}\right)\right) \longrightarrow k\left[\left[t_{0}, \ldots, t_{l-1}, t_{l+1}, \ldots, t_{r}\right]\right] \\
t_{i} \longmapsto t_{i} \quad \text { for } i \neq l \\
t_{i} \longmapsto 0 \quad \text { for } i=l
\end{aligned}
$$

Thus we have a morphism $\tilde{V}:=\amalg_{l=0}^{r} V_{l} \rightarrow V$. Let $Z^{\prime}:=\amalg_{l=0}^{r} Z_{l}$ and let $Z^{\prime} \rightarrow \tilde{V}$ be the morphism induced by the morphisms $Z_{l} \rightarrow V_{l}$. Let $V^{\prime \prime}:=\tilde{V} \times_{V} \tilde{V}$ and let $\operatorname{pr}_{\nu}: V^{\prime \prime} \rightarrow \tilde{V}$ be the projection onto the $\nu$-th component. Let $Z_{\nu}^{\prime \prime}:=Z^{\prime} \times_{\tilde{V}, \mathrm{pr}_{\nu}} V^{\prime \prime}$ be the pull-back of $Z^{\prime}$ by $\operatorname{pr}_{\nu}$. Then there is an isomorphism $Z_{1}^{\prime \prime} \stackrel{\sim}{\rightarrow} Z_{2}^{\prime \prime}$ over $V^{\prime \prime}$ which provides an effective descent datum for $Z^{\prime}$ relative to the morphism $\tilde{V} \rightarrow V$. Thus there exists a $V$-scheme $Z$ such that $Z^{\prime} \cong Z \times_{V} \tilde{V}$. The morphisms $Z_{l} \rightarrow C_{0} \times_{k} V_{l}$ induce a morphism $Z \rightarrow C_{0} \times_{k} V$ and it is clear that this defines a modification of $C_{0}$ over $V$. Furthermore, $Z \rightarrow C_{0} \times_{k} V$ is universal in the following sense: Let $S$ be the spectrum of a complete local $k$-algebra with residue field $k$ and let $\mathcal{C} \rightarrow C_{0} \times_{k} S$ be a modification of $C_{0}$ over $S$ such that its special fibre contains a chain of projective lines of length $r$ (and none of length $r+1$ ). Then there exists a morphism $S \rightarrow V$ such that $\mathcal{C} \cong Z \times_{V} S$ (cf. appendix in [NS]).

Finally we remark that the composed morphisms

$$
V_{l} \stackrel{\sigma_{l}}{\longrightarrow} Z_{l} \longrightarrow Z
$$

induce a closed immersion $\tilde{V} \hookrightarrow Z$ which identifies $\tilde{V}$ with the subscheme $\Sigma$ of $Z$ defined by the first Fitting ideal of $\Omega_{Z / V}^{1}$.

Proof of Theorem 4.9. Let $N \geq n$ and let $H_{N, 0} / k$ be the special fibre of the $B$ scheme $H_{N}$ defined in 3.20 . Let $\mathcal{C}_{H_{N, 0}} \hookrightarrow C_{0} \times_{\text {Spec }(k)} \operatorname{Grass}_{n}\left(k^{N}\right) \times_{\text {Spec }(k)} H_{N, 0}$ be the universal object over $H_{N, 0}$. Let $\widetilde{H_{N, 0}} \hookrightarrow \mathcal{C}_{H_{N, 0}}$ be the locus of points where the morphism $\mathcal{C}_{H_{N, 0}} \rightarrow H_{N, 0}$ fails to be smooth. More precisely, $\widetilde{H_{N, 0}}$ is the closed subscheme of $\mathcal{C}_{H_{N, 0}}$, defined by the first Fitting ideal of the sheaf $\Omega_{\mathcal{C}_{H_{N, 0}} / H_{N, 0}}^{1}$. I claim that $\widetilde{H_{N, 0}} \rightarrow H_{N, 0}$ is the normalization of $H_{N, 0}$. Indeed, let $x$ be a closed point of $H_{N, 0}$ and let $H_{x}$ be the spectrum of the completion of the local ring of $H_{N, 0}$ at $x$. Let $\mathcal{C} \rightarrow H_{x}$ be the pull-back of the universal curve $\mathcal{C}_{H_{N, 0}}$ by the natural map $H_{x} \rightarrow H_{N, 0}$. Let $\Sigma \hookrightarrow Z \rightarrow V$ be as in 4.11 with $r \geq 0$ chosen such that the special fibres of $\mathcal{C} \rightarrow H_{x}$ and $Z \rightarrow V$ be isomorphic. Then there is a morphism $H_{x} \rightarrow V$ such that $\mathcal{C}=Z \times_{V} H_{x}$ and it follows that $\widetilde{H_{N, 0}} \times{ }_{H_{N, 0}} H_{x}=\Sigma \times_{V} H_{x}$ 
Since furthermore the morphism $H_{x} \rightarrow V$ is formally smooth (cf. [NS], appendix), the claim now follows from Lemma 4.10 (observe that $H_{N, 0}$ is the disjoint union of schemes of finite type over $k$ ).

It suffices now to show that there exists a morphism $\widetilde{H_{N, 0}} \rightarrow \operatorname{GVBD}\left(\tilde{C}_{0}, p_{1}, p_{2}\right)$ such that the following diagram of $k$-groupoids is cartesian:

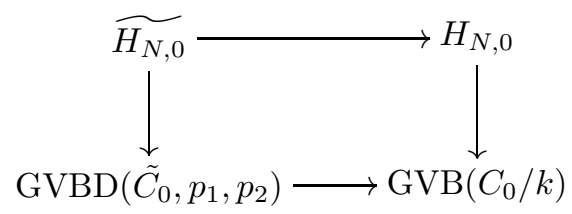

But this is easy: Let $S$ be an affine $k$-scheme. An $S$-valued point of $\widetilde{H_{N, 0}}$ is an $S$-valued point $\mathcal{C} \hookrightarrow C_{0} \times_{k} \times \operatorname{Grass}_{n}\left(k^{n}\right) \times{ }_{k} S$ of $H_{N, 0}$ plus a section $S \rightarrow \mathcal{C}$ of the projection $\pi: \mathcal{C} \rightarrow S$, meeting $\mathcal{C}$ in the nonsmooth locus of $\pi$. Observe that this is exactly the description of an $S$-valued point of the fibre product $\operatorname{GVBD}\left(\tilde{C}_{0}, p_{1}, p_{2}\right) \times_{\mathrm{GVB}\left(C_{0} / k\right)} H_{N, 0}$.

\section{Simple modifications of a Relative CuRve WITH A SMOOTH SECTION}

Definition 5.1. Let $S$ be a scheme and let $\pi: \mathcal{C} \rightarrow S$ be a flat morphism whose geometric fibers are connected and of dimension one. Let $s: S \rightarrow \mathcal{C}$ be a section of $\pi$ whose image lies in the subset of $\mathcal{C}$ where $\pi$ is smooth. A simple modification $\left(\mathcal{C}^{\prime}, f, \pi^{\prime}, s^{\prime}\right)$ of $(\mathcal{C}, \pi, s)$ is a diagram

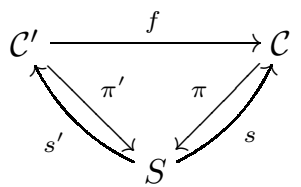

with the following properties:

(1) The morphism $\pi^{\prime}$ is again flat with connected geometric fibers of dimension one and $s^{\prime}$ is a section of $\pi^{\prime}$ whose image consists of points where $\pi^{\prime}$ is smooth.

(2) The diagram is commutative in the sense that $\pi \circ f=\pi^{\prime}$ and $f \circ s^{\prime}=s$.

(3) The morphism $f$ is proper and finitely presented.

(4) Let $z \in S$ be a point. Then there are two possibilities for the induced morphism $f_{z}: \mathcal{C}_{z}^{\prime} \rightarrow \mathcal{C}_{z}$ of fibres over $z$ : Either $f_{z}$ is an isomorphism, or $\mathcal{C}_{z}^{\prime}$ is the quotient of $R \cong \mathbb{P}_{\kappa(z)}^{1}$ and $\mathcal{C}_{z}$ by the identification of a point in $R(\kappa(z))$ with $s(z) \in \mathcal{C}_{z}$ and $f_{z}$ contracts $R$ to the point $s(z)$.

Construction 5.2. Let $\left(\mathcal{C}^{\prime}, f, \pi^{\prime}, s^{\prime}\right)$ be a simple modification of $(\mathcal{C}, \pi, s)$ as in Definition 5.1 and let $\mathcal{M}:=\mathcal{O}_{\mathcal{C}^{\prime}}\left(-s^{\prime}\right) \otimes f^{*} \mathcal{O}_{\mathcal{C}}(s)$. We have the following exact diagram of $\mathcal{O}_{\mathcal{C}^{\prime} \text {-modules: }}$

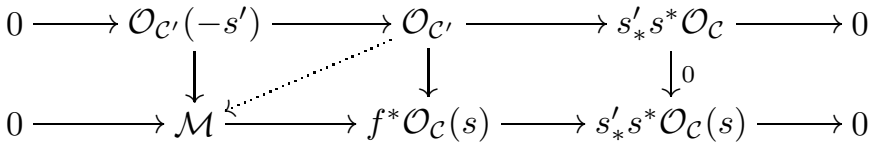

where the vertical arrows are induced by the morphism $\mathcal{O}_{\mathcal{C}^{\prime}} \rightarrow f^{*} \mathcal{O}_{\mathcal{C}}(s)$ obtained by applying $f^{*}$ to the natural injection $\mathcal{O}_{\mathcal{C}} \hookrightarrow \mathcal{O}_{\mathcal{C}}(s)$. Since the right vertical arrow 
obviously vanishes, the middle vertical arrow factorizes as indicated by the dotted arrow. Applying $\left(s^{\prime}\right)^{*}$ to the morphism $m: \mathcal{O}_{\mathcal{C}^{\prime}} \rightarrow \mathcal{M}$ thus obtained yields a morphism

$$
\mu_{1}: \mathcal{O}_{S} \rightarrow M_{1}:=\left(s^{\prime}\right)^{*} \mathcal{O}_{\mathcal{C}^{\prime}}\left(-s^{\prime}\right) \otimes s^{*} \mathcal{O}_{\mathcal{C}}(s) .
$$

Proposition 5.3. Let $S$ be an arbitrary scheme, $\pi: \mathcal{C} \rightarrow S$ a proper, finitely presented, flat morphism with connected geometric fibers of dimension one and let $s: S \rightarrow \mathcal{C}$ be a section of $\pi$ whose image consists of smooth points of $\pi$. Assume that for every point $z \in S$ we have $H^{0}\left(\mathcal{C}_{z}, \mathcal{O}_{\mathcal{C}_{z}}\right)=\kappa(z)$ and $H^{0}\left(\mathcal{C}_{z}, \mathcal{O}_{\mathcal{C}_{z}}(-s(z))\right)=(0)$, where $\mathcal{C}_{z}$ denotes the fibre of $\pi$ at $z$. Then the above Construction 5.2 yields an isomorphism of groupoids:

$$
\left\{\begin{array}{l}
\text { Simple modifications of }(\mathcal{C}, \pi, s) \\
\text { in the sense of Definition }[5.1
\end{array}\right\} \stackrel{\sim}{\rightarrow}\left\{\begin{array}{l}
\text { Pairs }(M, \mu) \text {, where } M \text { is } \\
\text { an invertible } \mathcal{O}_{S} \text {-module and } \\
\mu: \mathcal{O}_{S} \rightarrow M \text { is a global } \\
\text { section of } M
\end{array}\right\}
$$

In the remainder of this section we prove the above proposition. For this, we fix a scheme $S$ and data $(\mathcal{C}, \pi, s)$ as in the proposition.

Construction 5.4. To a pair $(M, \mu)$, consisting of an invertible $\mathcal{O}_{S}$-module $M$ together with a global section $\mu$ we associate a diagram

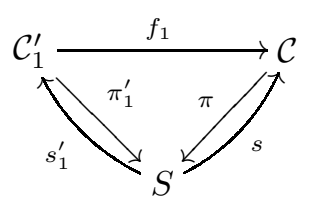

as follows:

Let $\mathcal{I}$ be the $\mathcal{O}_{\mathcal{C}}$-module defined by the exact sequence

$$
0 \rightarrow \pi^{*} M^{\vee} \otimes \mathcal{O}_{\mathcal{C}}(-s) \rightarrow \pi^{*} M^{\vee} \oplus \mathcal{O}_{\mathcal{C}}(-s) \rightarrow \mathcal{I} \rightarrow 0
$$

where $\pi^{*} M^{\vee} \otimes \mathcal{O}_{\mathcal{C}}(-s) \rightarrow \pi^{*} M^{\vee}$ is induced by the natural injection $\mathcal{O}_{\mathcal{C}}(-s) \rightarrow \mathcal{O}_{\mathcal{C}}$ and $\pi^{*} M^{\vee} \otimes \mathcal{O}_{\mathcal{C}}(-s) \rightarrow \mathcal{O}_{\mathcal{C}}(-s)$ is induced by $-\mu$. We define

$$
\mathcal{C}_{1}^{\prime}:=\operatorname{Proj}(\operatorname{Sym} \mathcal{I}) \text {. }
$$

Let $f_{1}: \mathcal{C}_{1}^{\prime} \rightarrow \mathcal{C}$ be the projection and $\pi_{1}^{\prime}:=\pi \circ f_{1}$. The morphism $s_{1}^{\prime}: S \rightarrow \mathcal{C}_{1}^{\prime}$ is the one induced by the invertible quotient $s^{*} \mathcal{I} \rightarrow M^{\vee}$ of $s^{*} \mathcal{I}$ which is adjoint to the arrow $\mathcal{I} \rightarrow s_{*} M^{\vee}$ defined by the exact diagram

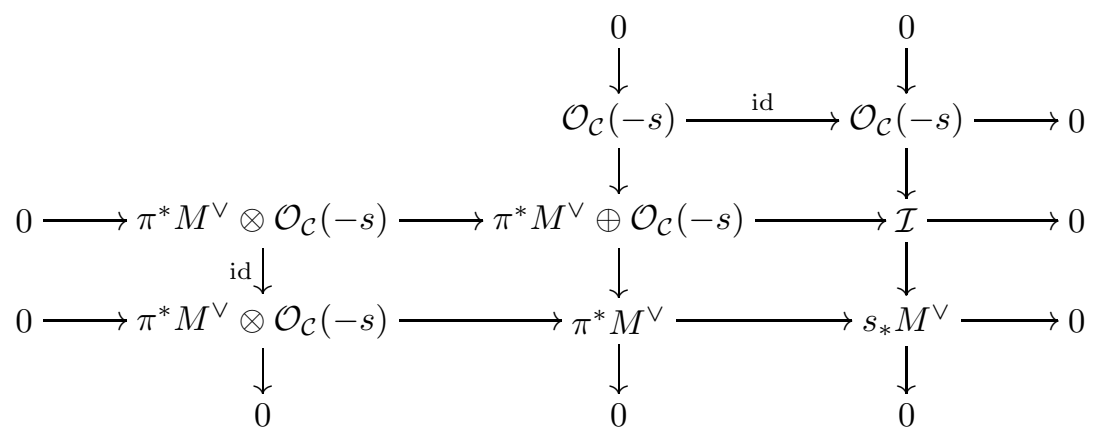


Lemma 5.5. Let $(\mathcal{C}, \pi, s)$ be as above. Assume that $S=\operatorname{Spec} A$ and $\mathcal{C}=\operatorname{Spec} B$ for a ring $A$ and an $A$-algebra $B$. Let $b \in B$ be a regular element that defines the section $s: S \rightarrow \mathcal{C}$. In particular, we have $B /(b)=A$ as $A$-algebras. Let $a \in A$ and let $\mathcal{I}$ be the $B$-module associated as in Construction 5.4 to the pair $(M, \mu):=(A, a)$. Then we have an isomorphism of graded B-algebras

$$
\operatorname{Sym}(\mathcal{I}) \cong B[X, Y] /(b X-a Y)
$$

and $\operatorname{Sym}(\mathcal{I})$ is flat over $A$.

Proof. By definition we have an exact sequence of $B$-modules:

$$
\begin{aligned}
0 \longrightarrow B & \longrightarrow B \oplus B \longrightarrow \mathcal{I} \longrightarrow 0 \\
1 & \longmapsto(b,-a)
\end{aligned}
$$

By [B], III.2, Prop.4, this induces an exact sequence of graded $B$-modules:

$$
\begin{gathered}
\operatorname{Sym}(B \oplus B)(-1) \longrightarrow \operatorname{Sym}(B \oplus B) \longrightarrow \operatorname{Sym}(\mathcal{I}) \longrightarrow 0 \\
f \longmapsto(b,-a) \cdot f
\end{gathered}
$$

This proves the required isomorphism. The flatness of $\operatorname{Sym}(\mathcal{I})$ now follows from the fact that the image of $b$ in $B /(\mathfrak{p})$ and thus the image of $b X-a Y$ in $B /(\mathfrak{p})[X, Y]$ is regular for any prime ideal $\mathfrak{p} \subset A$ (cf. [Ma], 22.6).

Lemma 5.6. The diagram

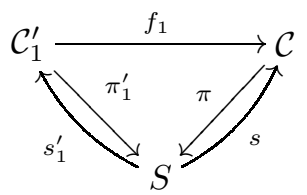

constructed in 5.4 is a simple modification of $(\mathcal{C}, \pi, s)$. Furthermore, Construction 5.4 commutes with base change $S^{\prime} \rightarrow S$.

Proof. It is clear that $f_{1}$ is proper and that $\pi \circ f_{1}=\pi_{1}^{\prime}, f_{1} \circ s_{1}^{\prime}=s, \pi_{1}^{\prime} \circ s_{1}^{\prime}=\mathrm{id}_{S}$. From the exact sequence $0 \rightarrow \mathcal{O}_{\mathcal{C}}(-s) \rightarrow \mathcal{I} \rightarrow s_{*} M^{\vee} \rightarrow 0$ it follows that $\mathcal{I}$ is invertible outside $s(S)$, thus $f_{1}$ is an isomorphism over the complement of the section $s$. The flatness of $\pi_{1}^{\prime}$ now follows easily from Lemma 5.5. Let $x \in s(S)$. By Lemma 5.5 we have $\mathcal{I}_{x} \cong \mathcal{O}_{\mathcal{C}, x}[X, Y] /(b X-a Y)$ for certain elements $a \in \mathcal{O}_{S, \pi(x)}$, $b \in \mathcal{O}_{\mathcal{C}, x}$. Therefore, if $f_{1}$ is not an isomorphism in any neighbourhood of $x$, then both $a$ and $b$ are in the maximal ideal of $\mathcal{O}_{\mathcal{C}, x}$. This implies $f^{-1}(x) \cong \mathbb{P}_{\kappa(x)}^{1}$. It is immediate that Construction 5.4 commutes with base change. An easy calculation in the case $S=\operatorname{Spec}(k)$, with $k$ algebraically closed, shows that the geometric fibers of $\pi_{1}^{\prime}$ are connected and of dimension one and that the image of $s_{1}^{\prime}$ consists of smooth points of $\pi_{1}^{\prime}$.

We want to show that under the assumptions of Proposition 5.3. Construction 5.4 is the inverse to Construction 5.2. For this, we start with a simple modification $\left(\mathcal{C}^{\prime}, f, \pi^{\prime}, s^{\prime}\right)$ of $(\mathcal{C}, \pi, s)$, apply Construction 5.2 to get a pair $\left(M_{1}, \mu_{1}\right)$ and then apply Construction 5.4 to $(M, \mu):=\left(M_{1}, \mu_{1}\right)$ to get a sheaf $\mathcal{I}$ and a simple modification $\left(\mathcal{C}_{1}^{\prime}, f_{1}, \pi_{1}^{\prime}, s_{1}^{\prime}\right)$ of $(\mathcal{C}, \pi, s)$, where $\mathcal{C}_{1}^{\prime}=\operatorname{Proj}(\operatorname{Sym}(\mathcal{I}))$. We have to show the existence of a canonical isomorphism $\mathcal{C}^{\prime} \stackrel{\sim}{\rightarrow} \mathcal{C}_{1}^{\prime}$ which is compatible with the respective projections and sections. This is done in Lemma 5.11 below. 
We need some preliminary results. Let $\mathcal{M}:=\mathcal{O}_{\mathcal{C}^{\prime}}\left(-s^{\prime}\right) \otimes f^{*} \mathcal{O}_{\mathcal{C}}(s)$. Since $\left(s^{\prime}\right)^{*} \mathcal{M}^{\vee}=M^{\vee}$, we have a canonical morphism $\mathcal{M}^{\vee} \rightarrow s_{*}^{\prime} M^{\vee}$. Application of $\pi_{*}^{\prime}$ yields a morphism $\pi_{*}^{\prime} \mathcal{M}^{\vee} \rightarrow M^{\vee}$.

Lemma 5.7. Let $(\mathcal{C}, \pi, s)$ satisfy the assumptions of Proposition 5.3 and let $\left(\mathcal{C}^{\prime}, f\right.$, $\left.\pi^{\prime}, s^{\prime}\right)$ be a simple modification of $(\mathcal{C}, \pi, s)$. Then the canonical morphism $\pi_{*}^{\prime} \mathcal{M}^{\vee} \rightarrow$ $M^{\vee}$ is an isomorphism.

Proof. The proof of this lemma will be given in section 6 .

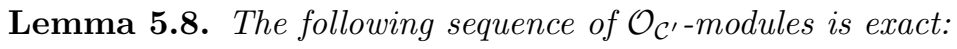

$$
0 \rightarrow\left(\pi^{\prime}\right)^{*} M^{\vee} \otimes \mathcal{O}_{\mathcal{C}^{\prime}}\left(-s^{\prime}\right) \rightarrow\left(\pi^{\prime}\right)^{*} M^{\vee} \oplus f^{*} \mathcal{O}_{\mathcal{C}}(-s) \rightarrow \mathcal{M}^{\vee} \rightarrow 0
$$

where the involved morphisms are defined as follows:

- $\left(\pi^{\prime}\right)^{*} M^{\vee} \otimes \mathcal{O}_{\mathcal{C}^{\prime}}\left(-s^{\prime}\right) \rightarrow\left(\pi^{\prime}\right)^{*} M^{\vee}$ is induced by $\mathcal{O}_{\mathcal{C}^{\prime}}\left(-s^{\prime}\right) \hookrightarrow \mathcal{O}_{\mathcal{C}^{\prime}}$.

- $\left(\pi^{\prime}\right)^{*} M^{\vee} \otimes \mathcal{O}_{\mathcal{C}^{\prime}}\left(-s^{\prime}\right) \rightarrow f^{*} \mathcal{O}_{\mathcal{C}}(-s)$ is induced by the negative of the adjoint of $M^{\vee} \stackrel{\sim}{\rightarrow} \pi_{*}^{\prime} \mathcal{M}^{\vee}$ (cf. Lemma [5.7).

- $\left(\pi^{\prime}\right)^{*} M^{\vee} \rightarrow \mathcal{M}^{\vee}$ is the adjoint of $M^{\vee} \stackrel{\sim}{\rightarrow} \pi_{*}^{\prime} \mathcal{M}^{\vee}$ (cf. Lemma 5.7).

- $f^{*} \mathcal{O}_{\mathcal{C}}(-s) \rightarrow \mathcal{M}^{\vee}$ is induced by $\mathcal{O}_{\mathcal{C}^{\prime}} \hookrightarrow \mathcal{O}_{\mathcal{C}^{\prime}}\left(s^{\prime}\right)$.

Proof. First of all, the sequence under consideration is a complex, as its middle part is of the shape

$$
N \otimes L_{1} \stackrel{(u,-v)}{\longrightarrow} N \oplus L_{2} \stackrel{u+v}{\longrightarrow} L_{1}^{\vee} \otimes L_{2} \quad,
$$

where $N, L_{1}, L_{2}$ are invertible sheaves and $u: L_{1} \rightarrow \mathcal{O}$ and $v: N \rightarrow L_{1}^{\vee} \otimes L_{2}$ are morphisms. Since all the sheaves occuring in the sequence are locally free and the rank of the sheaf in the middle is the sum of the ranks of the two other sheaves, it suffices to show for each point $x \in \mathcal{C}^{\prime}$ the injectivity of $\alpha_{x}$ and the surjectivity of $\beta_{x}$, where

$$
\begin{aligned}
\alpha_{x} & : \quad\left(\left(\pi^{\prime}\right)^{*} M^{\vee} \otimes \mathcal{O}_{\mathcal{C}^{\prime}}\left(-s^{\prime}\right)\right)[x] \rightarrow\left(\left(\pi^{\prime}\right)^{*} M^{\vee} \oplus f^{*} \mathcal{O}_{\mathcal{C}}(-s)\right)[x] \\
\beta_{x} & : \quad\left(\left(\pi^{\prime}\right)^{*} M^{\vee} \oplus f^{*} \mathcal{O}_{\mathcal{C}}(-s)\right)[x] \rightarrow \mathcal{M}^{\vee}[x]
\end{aligned}
$$

are the induced morphisms on the fibers of the sheaves at $x$. In particular, we can assume that $S=\operatorname{Spec}(k)$ for a field $k$. If $f$ is an isomorphism, the statement is easy to see. Otherwise, $\mathcal{C}^{\prime}=R \cup \mathcal{C}$ and $R \cap \mathcal{C}=\{s\}$, where $R \cong \mathbb{P}_{k}^{1}$ and three cases are possible: Either $x \in \mathcal{C}$, or $x \in R \backslash\left\{s, s^{\prime}\right\}$, or $x=s^{\prime}$. It is not difficult to check the statement in each of these cases.

Lemma 5.9. (1) We have $R^{1} f_{*} \mathcal{O}_{\mathcal{C}^{\prime}}\left(-s^{\prime}\right)=0$.

(2) The adjunction morphism $\mathcal{O}_{\mathcal{C}} \rightarrow f_{*} \mathcal{O}_{\mathcal{C}^{\prime}}$ is an isomorphism.

(3) The morphism $\mathcal{O}_{\mathcal{C}}(-s) \rightarrow f_{*} \mathcal{O}_{\mathcal{C}^{\prime}}\left(-s^{\prime}\right)$ adjoint to the morphism $f^{*} \mathcal{O}_{\mathcal{C}}(-s) \rightarrow$ $\mathcal{O}_{\mathcal{C}^{\prime}}\left(-s^{\prime}\right)$ (cf. Construction 5.2) is an isomorphism.

(4) We have $\pi_{*} \mathcal{O}_{\mathcal{C}}=\pi_{*}^{\prime} \mathcal{O}_{\mathcal{C}^{\prime}}=\mathcal{O}_{S}$.

Proof. Since obviously $H^{1}\left(f^{-1}(x), \mathcal{O}_{\mathcal{C}^{\prime}}\left(-s^{\prime}\right) \otimes_{\mathcal{O}_{\mathcal{C}}} \kappa(x)\right)=0$ for every point $x \in$ $\mathcal{C}$, Corollary 1.5 in $\mathrm{Kn}$ implies the first assertion in the lemma. Similarly, it follows from loc. cit. that $f_{*} \mathcal{O}_{\mathcal{C}^{\prime}}$ is flat over $S$ and that for every $z \in S$ we have $f_{*} \mathcal{O}_{\mathcal{C}^{\prime}} \otimes_{\mathcal{O}_{S}} \kappa(z)=\left(f_{z}\right)_{*} \mathcal{O}_{\mathcal{C}_{z}^{\prime}}$, where $f_{z}: \mathcal{C}_{z}^{\prime} \rightarrow \mathcal{C}_{z}$ is the induced morphism between the fibers over $z$. With the help of Nakayama's lemma, this implies the second 
assertion. For the third assertion it suffices to prove the commutativity of the diagram

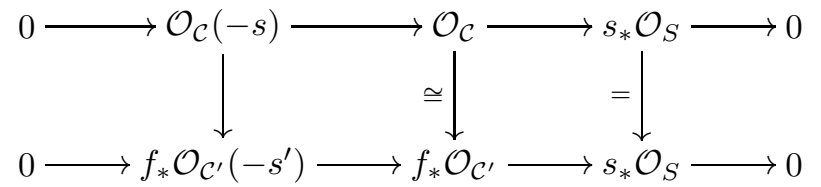

where the middle vertical arrow is the isomorphism from (2) and the lower sequence comes from applying $f_{*}$ to the exact sequence $0 \rightarrow \mathcal{O}_{\mathcal{C}^{\prime}}\left(-s^{\prime}\right) \rightarrow \mathcal{O}_{\mathcal{C}^{\prime}} \rightarrow s_{*}^{\prime} \mathcal{O}_{S} \rightarrow 0$. The right square in this diagram is obviously commutative. The commutativity of the left square is equivalent to the commutativity of the adjoint square

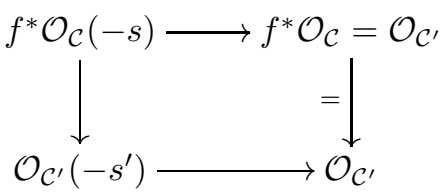

But this square commutes by Construction 5.2. By [EGA III], (7.8.8), the equality $\pi_{*} \mathcal{O}_{\mathcal{C}}=\mathcal{O}_{S}$ follows from the assumption $H^{0}\left(\mathcal{C}_{z}, \mathcal{O}_{\mathcal{C}_{z}}\right)=\kappa(z)$ for all $z$. The assertion $\pi_{*}^{\prime} \mathcal{O}_{\mathcal{C}^{\prime}}=\mathcal{O}_{S}$ follows now from $(2)$.

Lemma 5.10. There exists a canonical isomorphism $\mathcal{I} \stackrel{\sim}{\rightarrow} f_{*} \mathcal{M}^{\vee}$.

Proof. Consider the following diagram of $\mathcal{O}_{\mathcal{C}}$-modules:

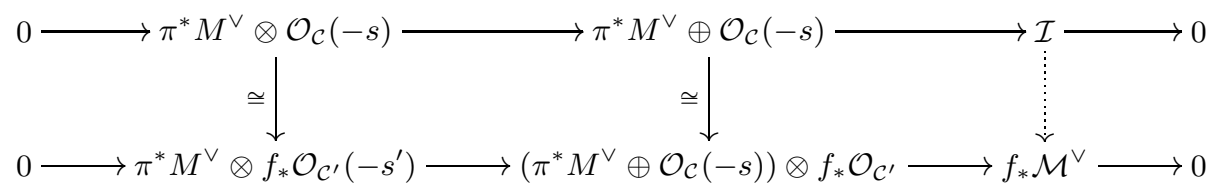

where the first row is the defining exact sequence for $\mathcal{I}$ (cf. Construction 5.4) and the second row comes from applying $f_{*}$ to the exact sequence of Lemma 5.8. It is exact by Lemma 5.9.11). The left vertical arrow is induced by the isomorphism in 5.9(3), the middle vertical arrow by the one in 5.9(2). The lemma follows, if we can show the commutativity of the left square in this diagram. We give the details, since at some point we make use of the equality $\pi_{*} \mathcal{O}_{\mathcal{C}}=\mathcal{O}_{S}$ and thus of the assumption $H^{0}\left(\mathcal{C}_{z}, \mathcal{O}_{\mathcal{C}_{z}}\right)=\kappa(z)$. The proof of the commutativity of the left square in the above diagram amounts to showing that the two squares
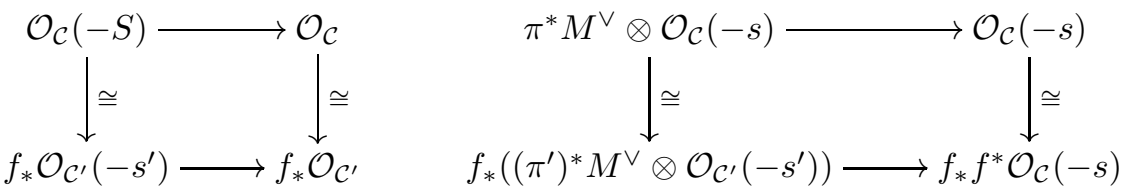

are both commutative. For the left square this has been done already in the proof of Lemma 5.9. The commutativity of the right square is equivalent to the commutativity of the adjoint square

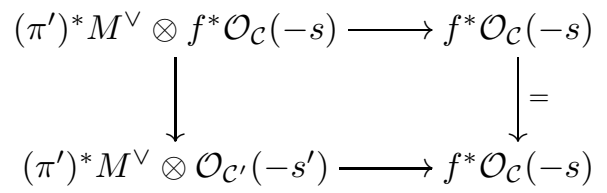


which in turn is equivalent to the commutativity of

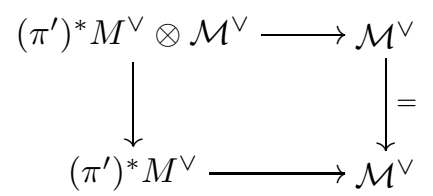

where the upper horizontal and the left vertical arrows are induced by $\mathcal{O}_{S} \rightarrow M$ and $\mathcal{O}_{\mathcal{C}^{\prime}} \rightarrow \mathcal{M}$ respectively, which were constructed in 5.2 and the lower horizontal arrow is the adjoint of the inverse of the isomorphism $\pi_{*}^{\prime} \mathcal{M}^{\vee} \stackrel{\sim}{\rightarrow} M^{\vee}$ from Lemma 5.7. To prove the commutativity of $(*)$, observe first that by general nonsense the following diagram commutes:

$(* *)$

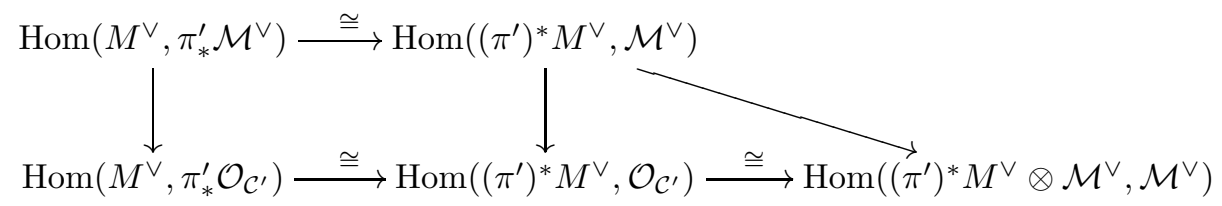

where the vertical arrows and the oblique arrow are all induced by $\mathcal{O}_{\mathcal{C}^{\prime}} \rightarrow \mathcal{M}$ from 5.2. The commutativity of $(*)$ follows, if we can show that the left vertical arrow of $(* *)$ maps the inverse of the isomorphism $\pi_{*}^{\prime} \mathcal{M}^{\vee} \stackrel{\sim}{\rightarrow} M^{\vee}$ from Lemma 5.7 to the morphism $M^{\vee} \rightarrow \pi_{*}^{\prime} \mathcal{O}_{\mathcal{C}^{\prime}}$ obtained by composing $\mu: M^{\vee} \rightarrow \mathcal{O}_{S}$ from 5.2 with the canonical morphism $a: \mathcal{O}_{S} \rightarrow \pi_{*}^{\prime} \mathcal{O}_{\mathcal{C}^{\prime}}$. This amounts to showing the commutativity of the diagram

$(* * *)$

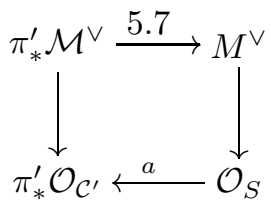

Now consider the two squares
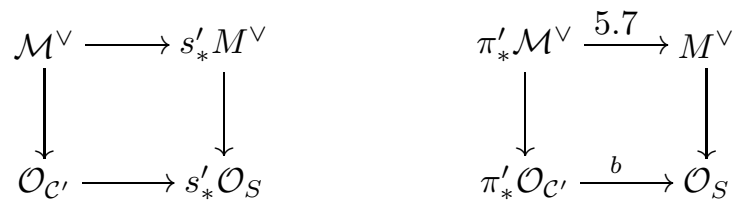

The left square is obviously commutative, therefore so is the right square, which is the image of the left one under the functor $\pi_{*}^{\prime}$. Since by Lemma 5.9) the morphisms $a$ and $b$ are inverse to each other (this is the point where the equality $\pi_{*}\left(\mathcal{O}_{\mathcal{C}}\right)=\mathcal{O}_{S}$ enters $)$, the commutativity of $(* * *)$ follows and we are done.

By $\underline{\mathrm{Kn}}$, Cor. 1.5 , the morphism $f^{*} \mathcal{I} \rightarrow \mathcal{M}^{\vee}$ adjoint to the isomorphism $\mathcal{I} \stackrel{\sim}{\rightarrow}$ $f_{*} \mathcal{M}^{\vee}$ is an epimorphism.

Lemma 5.11. The morphism $\mathcal{C}^{\prime} \rightarrow \mathcal{C}_{1}^{\prime}$ induced by the invertible quotient $f^{*} \mathcal{I} \rightarrow$ $\mathcal{M}^{\vee}$ of $f^{*} \mathcal{I}$ defined above is an isomorphism of $\mathcal{C}$-schemes. Furthermore, the following diagram commutes:

$$
\mathcal{C}^{\prime} \underset{s^{\prime}}{\underset{s_{1}^{\prime}}{\longrightarrow}} \mathcal{C}_{1}^{\prime}
$$


Proof. It is clear that $\mathcal{C}^{\prime} \rightarrow \mathcal{C}_{1}^{\prime}$ is a morphism of $\mathcal{C}$-schemes. To show that this is in fact an isomorphism, we first assume that $S=\operatorname{Spec}(k)$ for some field $k$. If $f$ is an isomorphism, then $\mathcal{I}$ is invertible by 5.10 and therefore $f_{1}: \mathcal{C}_{1}^{\prime} \rightarrow \mathcal{C}$ and $\mathcal{C}^{\prime} \rightarrow \mathcal{C}_{1}^{\prime}$ are isomorphisms. Otherwise, $\mathcal{C}^{\prime}$ is the glueing together of $R \cong \mathbb{P}_{k}^{1}$ and $\mathcal{C}$ by identifying a point in $R(k)$ with the point $s \in \mathcal{C}(k)$. Since both $f$ and $f_{1}$ are isomorphisms over the complement of $s$, we may assume that $\mathcal{C}$ is the spectrum of a discrete valuation ring with closed point $s$. It is now easy to see that the surjection $f^{*} \mathcal{I} \stackrel{\sim}{\rightarrow} f^{*} f_{*} \mathcal{M}^{\vee} \rightarrow \mathcal{M}^{\vee}$ induces an isomorphism

$$
\mathcal{C}^{\prime} \stackrel{\sim}{\rightarrow} \operatorname{Proj}\left(\operatorname{Sym}\left(f^{*} f_{*} \mathcal{M}^{\vee}\right)\right) \stackrel{\sim}{\rightarrow} \operatorname{Proj}(\operatorname{Sym}(\mathcal{I}))=\mathcal{C}_{1}^{\prime} .
$$

Now let $S$ be arbitrary. Since both $\mathcal{C}^{\prime}$ and $\mathcal{C}_{1}^{\prime}$ are flat over $S$ and all constructions commute with base change, it follows from the special case that $\mathcal{C}^{\prime} \rightarrow \mathcal{C}_{1}^{\prime}$ is an isomorphism in general. It remains to show that $s_{1}^{\prime}$ is the composition of $s^{\prime}$ with $\mathcal{C}^{\prime} \rightarrow \mathcal{C}_{1}^{\prime}$. For this, it suffices to show that the morphism $s^{*} \mathcal{I} \rightarrow M^{\vee}$ which one obtains by applying $\left(s^{\prime}\right)^{*}$ to the morphism $f^{*} \mathcal{I} \rightarrow \mathcal{M}^{\vee}$ coincides with the morphism $s^{*} \mathcal{I} \rightarrow M^{\vee}$ defining $s_{1}^{\prime}$ (cf. Construction 5.4). This follows formally by the yoga of pairs of adjoint morphisms.

Conversely, let a pair $(M, \mu)$ consisting of an invertible $\mathcal{O}_{S}$-module $M$ and a global section $\mu$ of $M$ be given. Applying Construction 5.4 to this pair, we get an $\mathcal{O}_{\mathcal{C}}$-module $\mathcal{I}$ and a simple modification $\left(\mathcal{C}_{1}^{\prime}, f_{1}, \pi_{1}^{\prime}, s_{1}^{\prime}\right)$ of $(\mathcal{C}, \pi, s)$, where $\mathcal{C}_{1}^{\prime}=$ Proj $(\operatorname{Sym}(\mathcal{I}))$. Construction 5.2 applied to the simple modification $\left(\mathcal{C}^{\prime}, f, \pi^{\prime}, s^{\prime}\right):=$ $\left(\mathcal{C}_{1}^{\prime}, f_{1}, \pi_{1}^{\prime}, s_{1}^{\prime}\right)$ yields a pair $\left(M_{1}, \mu_{1}\right)$. We have to establish a canonical isomorphism $M \stackrel{\sim}{\rightarrow} M_{1}$ which makes the respective global sections correspond.

Let $\mathcal{S}$ be the graded $\mathcal{O}_{\mathcal{C}}$-module $\operatorname{Sym}(\mathcal{I})$. By definition, the section $s^{\prime}: S \rightarrow \mathcal{C}^{\prime}$ is given by applying Proj to the surjection $\mathcal{S} \rightarrow \operatorname{Sym}\left(s_{*} M^{\vee}\right)$ which is induced by the epimorphism $\mathcal{I} \rightarrow s_{*} M^{\vee}$ defined in Construction [5.4. Therefore, we have $\left(s^{\prime}\right)^{*} \mathcal{O}_{\mathcal{C}^{\prime}}(1)=\left(\mathcal{S}(1) \otimes_{\mathcal{S}} \operatorname{Sym}\left(s_{*} M^{\vee}\right)\right)^{\sim}$, where $\mathcal{O}_{\mathcal{C}^{\prime}}(1)$ denotes the tautological invertible sheaf on $\mathcal{C}^{\prime}=\operatorname{Proj}(\operatorname{Sym}(\mathcal{I}))$ (cf. [EGA II], (3.2.5.1)) and where $\sim$ means taking the quasicoherent sheaf on $S=\operatorname{Proj}\left(\operatorname{Sym}\left(s_{*} M^{\vee}\right)\right)$ associated to a graded $\operatorname{Sym}\left(s_{*} M^{\vee}\right)$-module. It is easy to see that the sheaf $\left(\mathcal{S}(1) \otimes_{\mathcal{S}} \operatorname{Sym}\left(s_{*} M^{\vee}\right)\right)^{\sim}$ is just $M^{\vee}$. Thus we have

$$
\left(s^{\prime}\right)^{*} \mathcal{O}_{\mathcal{C}^{\prime}}(1)=M^{\vee}
$$

From the exact sequence of $\mathcal{O}_{\mathcal{C}}$-modules $0 \rightarrow \mathcal{O}_{\mathcal{C}}(-s) \rightarrow \mathcal{I} \rightarrow s_{*} M^{\vee} \rightarrow 0$ (cf. Construction 5.4) we get by $\mathrm{B}$, III.2 Prop.4, the exact sequence of graded $\mathcal{O}_{\mathcal{C}^{-}}$ modules

$$
\mathcal{S}(-1) \otimes_{\mathcal{O}_{\mathcal{C}}} \mathcal{O}_{\mathcal{C}}(-s) \rightarrow \mathcal{S} \rightarrow \operatorname{Sym}\left(s_{*} M^{\vee}\right) \rightarrow 0
$$

From Lemma 5.5 it follows easily that the left arrow is injective. Since obviously $\mathcal{O}_{\mathcal{C}^{\prime}}\left(-s^{\prime}\right)$ is the $\mathcal{O}_{\mathcal{C}^{\prime}}$-module associated to the graded $\mathcal{S}$-module

$$
\operatorname{ker}\left(\mathcal{S} \rightarrow \operatorname{Sym}\left(s_{*} M^{\vee}\right)\right),
$$

we obtain a canonical isomorphism

$$
\mathcal{O}_{\mathcal{C}^{\prime}}\left(s^{\prime}\right) \otimes f^{*} \mathcal{O}_{\mathcal{C}}(-s) \stackrel{\sim}{\rightarrow} \mathcal{O}_{\mathcal{C}^{\prime}}(1)
$$

Applying $\left(s^{\prime}\right)^{*}$ to it yields the required isomorphism $M \stackrel{\sim}{\rightarrow} M_{1}$.

Lemma 5.12. The isomorphism $M \stackrel{\sim}{\rightarrow} M_{1}$ maps the global section $\mu$ of $M$ to the global section $\mu_{1}$ of $M_{1}$. 
Proof. Since both Construction 5.2 and Construction 5.4 commute with base change $S^{\prime} \rightarrow S$ and since furthermore the definition of the isomorphism $M \stackrel{\sim}{\rightarrow} M_{1}$ depends only on a neighbourhood of the section $s$ in $\mathcal{C}$, we may assume $S=\operatorname{Spec}(A)$ and $\mathcal{C}=\operatorname{Spec}(B)$ for a local ring $A$ and local $A$-algebra $B$. Let $l: M \stackrel{\sim}{\rightarrow} A$ be an isomorphism. Let $a:=l(\mu)$ and $b$ a generator of the kernel $N$ of the surjection $B \rightarrow A$ associated to the section $s: S \rightarrow \mathcal{C}$. By Lemma 5.5 we may identify the graded $B$ module $\mathcal{S}$ with $B[X, Y] /(b X-a Y)$. Let $\mathcal{J}$ be the graded $\mathcal{S}$-module $\operatorname{ker}\left(\mathcal{S} \rightarrow \operatorname{Sym}\left(M^{\vee}\right)\right)$. It is generated by the element $Y \in \mathcal{S}$. By definition, we have

$$
M_{1}=\left(\mathcal{J} \otimes_{\mathcal{S}} \operatorname{Sym}\left(M^{\vee}\right)\right)_{(l)} \otimes_{A} N^{\vee},
$$

where the index $(l)$ means taking the zero-degree part of the localization by powers of $l \in \operatorname{Sym}\left(M^{\vee}\right)$ (cf. [EGA II], (2.2.1)). It is easy to check that the isomorphism $M \rightarrow M_{1}$ is given by

$$
\xi \mapsto \frac{Y \otimes l(\xi)}{l} \otimes b^{\vee},
$$

where $b^{\vee} \in N^{\vee}$ denotes the generator dual to $b$. On the other hand, it follows directly from the definitions that

$$
\mu_{1}=\frac{b X \otimes 1}{l} \otimes b^{\vee} .
$$

Therefore the statement follows from the equality $b X=a Y$ which holds in $\mathcal{S}$.

This completes the proof of Proposition 5.3 .

\section{Proof of Lemma 5.7}

Lemma 6.1. Lemma 5.7 is true in the case where $S$ is the spectrum of a field $k$.

Proof. If $f$ is an isomorphism, then $\mathcal{M}^{\vee}=\mathcal{O}_{\mathcal{C}}$ and the lemma follows directly from the assumption $H^{0}\left(\mathcal{C}, \mathcal{O}_{\mathcal{C}}\right)=k$. Otherwise, $\mathcal{C}^{\prime}$ is the union of a projective line $R \cong \mathbb{P}^{1}$ and the curve $\mathcal{C}$, and we have $R \cap \mathcal{C}=\{s\}$. Furthermore, the restriction of $f$ to $R$ is the constant map onto the point $s \in \mathcal{C}$ and the restriction of $f$ to $\mathcal{C} \subset \mathcal{C}^{\prime}$ is the identity on $\mathcal{C}$. Now we have

$$
\begin{aligned}
H^{0}\left(\mathcal{C}^{\prime}, \mathcal{M}^{\vee}\right) & =H^{0}\left(R,\left.\mathcal{M}^{\vee}\right|_{R}\right) \times_{\mathcal{M}^{\vee}[s]} H^{0}\left(\mathcal{C},\left.\mathcal{M}^{\vee}\right|_{\mathcal{C}}\right) \\
& =H^{0}\left(R, \mathcal{O}_{R}\left(s^{\prime}\right) \otimes_{k} s^{*} \mathcal{O}_{\mathcal{C}}(-s)\right) \times_{\mathcal{M}^{\vee}[s]}(0) \\
& =H^{0}\left(R, \mathcal{O}_{R}\left(s^{\prime}-s\right)\right) \otimes_{k} s^{*} \mathcal{O}_{\mathcal{C}}(-s)
\end{aligned}
$$

and the morphism $H^{0}\left(\mathcal{C}^{\prime}, \mathcal{M}^{\vee}\right) \rightarrow M^{\vee}$ is induced by the isomorphism

$$
H^{0}\left(R, \mathcal{O}_{R}\left(s^{\prime}-s\right)\right) \stackrel{\sim}{\rightarrow}\left(s^{\prime}\right)^{*} \mathcal{O}_{R}\left(s^{\prime}\right)=\left(s^{\prime}\right)^{*} \mathcal{O}_{\mathcal{C}^{\prime}}\left(s^{\prime}\right)
$$

(restriction to the point $s^{\prime}$ ).

Lemma 6.2. Let $k$ be a field and let $\tilde{f}_{\text {vers }}: \tilde{\mathcal{C}}_{\text {vers }}^{\prime} \rightarrow \tilde{\mathcal{C}}_{\text {vers }}$ be the blowing up of $\tilde{\mathcal{C}}_{\text {vers }}:=\operatorname{Spec}(k[[t]][[z]])$ in the closed point $t=z=0$. Let $\tilde{\mathcal{C}}_{\text {vers }}^{\prime} \rightarrow \operatorname{Spec}(k[[t]])$ be the obvious projection morphism. Denote by $\tilde{\mathcal{C}}_{0}^{\prime}$ the special fibre of this morphism. Then $\tilde{\mathcal{C}_{0}^{\prime}}$ has two irreducible components $R \cong \mathbb{P}_{k}^{1}$ and $\tilde{\mathcal{C}_{0}} \cong \operatorname{Spec}(k[[z]])$ which meet in a $k$-rational ordinary double point of $\tilde{\mathcal{C}}_{0}^{\prime}$. Furthermore, $\tilde{\mathcal{C}}_{\text {vers }}^{\prime}$ over the base $\left.\operatorname{Spec}(k[t]]\right)$ is a versal deformation of $\tilde{\mathcal{C}}_{0}^{\prime}$. 
Proof. It is clear that the special fibre $\tilde{\mathcal{C}}_{0}^{\prime}$ of $\tilde{\mathcal{C}}_{\text {vers }}^{\prime} \rightarrow \operatorname{Spec}(k[[t]])$ has the indicated shape. To show that $\tilde{\mathcal{C}}_{\text {vers }}^{\prime}$ over $\operatorname{Spec}(k[[t]])$ is a versal deformation of its special fibre, it suffices to prove that $\operatorname{Ext}^{2}\left(\Omega_{\tilde{\mathcal{C}}_{0}^{\prime} / k}^{1}, \mathcal{O}_{\tilde{\mathcal{C}}_{0}^{\prime}}\right)=(0)$ and $\operatorname{Ext}^{1}\left(\Omega_{\tilde{\mathcal{C}}_{0}^{\prime} / k}^{1}, \mathcal{O}_{\tilde{\mathcal{C}}_{0}^{\prime}}\right) \cong k$ and that the deformation $\tilde{\mathcal{C}}_{\text {vers }}^{\prime}$ has nonvanishing Kodaira-Spencer class. We identify $\tilde{\mathcal{C}}_{0}^{\prime}$ with the closed subscheme of $\mathbb{P}_{k[[z]]}^{1}=\operatorname{Proj}(k[[z]][u, v])$ defined by the equation $z . u$. Let $I \subset \mathcal{O}_{\mathbb{P}_{k[z]]}^{1}}$ be the defining sheaf of ideals. Then we have an exact sequence

$$
0 \longrightarrow I / I^{2} \longrightarrow \Omega_{\mathbb{P}_{k[z]]}^{1}}^{1} /\left.k\right|_{\tilde{\mathcal{C}}_{0}^{\prime}} \longrightarrow \Omega_{\tilde{\mathcal{C}}_{0}^{\prime} / k}^{1} \longrightarrow 0
$$

which is a resolution of $\Omega_{\tilde{\mathcal{C}}_{0}^{\prime} / k}^{1}$ by locally free $\mathcal{O}_{\tilde{\mathcal{C}}_{0}^{\prime}}$-modules. Applying the functor $\operatorname{Hom}\left(\cdot, \mathcal{O}_{\tilde{\mathcal{C}}_{0}^{\prime}}\right)$ to this sequence, we obtain a long exact sequence of Ext-groups. It is easy to see that for $\mathcal{F}=\Omega_{\mathbb{P}_{k[z]]}^{1} / k}^{1}||_{\tilde{\mathcal{C}}_{0}^{\prime}}$ or $\mathcal{F}=I / I^{2}$ we have $\operatorname{Ext}^{i}\left(\mathcal{F}, \mathcal{O}_{\tilde{\mathcal{C}}_{0}^{\prime}}\right)=$ $H^{i}\left(\tilde{\mathcal{C}_{0}^{\prime}}, \mathcal{F}^{\vee}\right)=(0)$ for every $i \geq 1$. Therefore $\operatorname{Ext}^{2}\left(\Omega_{\tilde{\mathcal{C}}_{0}^{\prime} / k}^{1}, \mathcal{O}_{\tilde{\mathcal{C}}_{0}^{\prime}}\right)$ vanishes and $\operatorname{Ext}^{1}\left(\Omega_{\tilde{\mathcal{C}}_{0}^{\prime} / k}^{1}, \mathcal{O}_{\tilde{\mathcal{C}}_{0}^{\prime}}\right)$ is the cokernel of the morphism $H^{0}\left(\tilde{\mathcal{C}}_{0}^{\prime},\left(\Omega_{\mathbb{P}_{k[z]]}^{1}}^{1} / k \mid \tilde{\mathcal{C}}_{0}^{\prime}\right)^{\vee}\right) \rightarrow$ $H^{0}\left(\tilde{\mathcal{C}}_{0}^{\prime},\left(I / I^{2}\right)^{\vee}\right)$ which in turn is easily seen to be one-dimensional. By inspection, one also shows that the Kodaira-Spencer class of the deformation $\tilde{\mathcal{C}}_{\text {vers }}^{\prime}$ is nonzero.

Lemma 6.3. Let $k$ be a field, let $A$ be a local artinian $k$-algebra with residue field $k$ and let $S:=\operatorname{Spec}(A)$. Let $\tilde{\mathcal{C}}:=\operatorname{Spec}(A[[z]])$ and let $\tilde{s}: S \rightarrow \tilde{\mathcal{C}}$ be the section $z=0$ of the structure morphism $\tilde{\pi}: \tilde{\mathcal{C}} \rightarrow S$. Let $\left(\tilde{\mathcal{C}}^{\prime}, \tilde{f}, \tilde{\pi}^{\prime}, \tilde{s}^{\prime}\right)$ be an arbitrary simple modification of $(\tilde{\mathcal{C}}, \tilde{\pi}, \tilde{s})$ such that $\tilde{f}$ is not an isomorphism. Then there is a morphism $S \rightarrow \operatorname{Spec}(k[[t]])$ such that the diagram

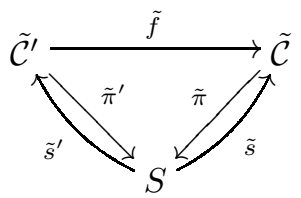

is isomorphic to the diagram which is induced by the base change $S \rightarrow \operatorname{Spec}(k[[t]])$ from the diagram

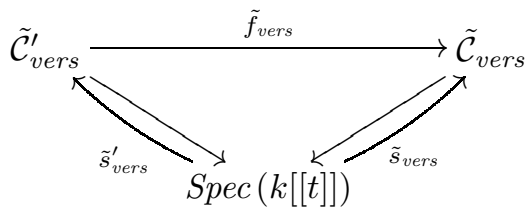

where $\tilde{s}_{\text {vers }}$ is the section $z=0$ of $\tilde{\mathcal{C}}_{\text {vers }} \rightarrow \operatorname{Spec}(k[[t]])$ and $\tilde{s}_{\text {vers }}^{\prime}$ is the proper transform of $\tilde{s}_{\text {vers }}$.

Proof. By Lemma 6.2, there is a morphism $S \rightarrow \operatorname{Spec}(k[[t]])$ such that $\tilde{\mathcal{C}}^{\prime}$ is isomorphic to $\tilde{\mathcal{C}}_{\text {vers }} \times_{\operatorname{Spec}(k[[t]])} S$. Thus there exists an open affine covering $\tilde{\mathcal{C}}^{\prime}=U_{1} \cup U_{2}$ with $U_{1}:=\operatorname{Spec}\left(B_{A}[u] /(u z-a)\right)$ and $U_{2}:=\operatorname{Spec}\left(B_{A}[v] /(z-v a)\right)$, where $B_{A}:=$ $A[[z]]$ and $a \in \mathfrak{m}_{A}$ is the image of $t$ by the morphism $k[[t]] \rightarrow A$. The glueing of $U_{1}$ 
and $U_{2}$ is given by:

$$
\operatorname{Spec}\left(B_{A}[u] /(u z-a)\right)_{(u)} \cong v
$$

The section $\tilde{s}^{\prime}: S \rightarrow \tilde{\mathcal{C}}^{\prime}$ factorizes through $U_{2}$ and is given by an $A$-morphism $\mathcal{O}_{U_{2}} \rightarrow A, v \mapsto \alpha$. We want to show that there exists an automorphism $\varphi^{\prime}$ of $\tilde{\mathcal{C}}^{\prime}$ such that the composition $\varphi^{\prime} \circ \tilde{s}^{\prime}$ is given by the $A$-morphism $\mathcal{O}_{U_{2}} \rightarrow A, v \mapsto 0$. By a standard argument we may assume $\alpha \mathfrak{m}_{A}=(0)$. Let $\varphi^{\prime}$ be defined on the affine pieces $U_{i}$ by the $B_{A}$-morphisms:

$$
\begin{array}{rlrl}
\mathcal{O}_{U_{1}} \stackrel{\cong}{\longmapsto} \mathcal{O}_{U_{1}} & \text { and } & \mathcal{O}_{U_{2}} \stackrel{\cong}{\longmapsto} \mathcal{O}_{U_{2}} \\
u \longmapsto & \longmapsto & & \\
u & & & \longmapsto-\alpha
\end{array}
$$

The automorphism $\varphi^{\prime}$ has the required property. Therefore we may assume that $\alpha=0$, i.e. that $\tilde{s}^{\prime}$ is induced by base change from $\tilde{s}_{\text {vers }}^{\prime}$. The morphism $\tilde{f}$ is given by $A$-morphisms

$$
\begin{array}{rlrl}
B_{A} \longrightarrow \mathcal{O}_{U_{1}} & \text { and } & B_{A} \longrightarrow \mathcal{O}_{U_{2}} \\
z & \longmapsto+p & z & \longmapsto
\end{array}
$$

where $p \in \mathfrak{m}_{A} \mathcal{O}_{U_{1}}$ and $q \in \mathfrak{m}_{A} \mathcal{O}_{U_{2}}$. Again we may assume that $\mathfrak{m}_{A} p=(0)=\mathfrak{m}_{A} q$. The constraint $\tilde{s}=\tilde{f} \circ \tilde{s}^{\prime}$ and compatibility with the glueing morphism over $U_{1} \cap U_{2}$ has the consequence that in fact $q=0$ and $p=z p_{1}$ for some $p_{1} \in \mathfrak{m}_{A} B_{A}$ with $\mathfrak{m}_{A} p_{1}=(0)$. We define an automorphism $\varphi$ of $\tilde{\mathcal{C}}=\operatorname{Spec}\left(B_{A}\right)$ by $z \mapsto\left(1-p_{1}\right) z$. Then the composed morphism $\varphi \circ \tilde{f}: \tilde{\mathcal{C}} \rightarrow \tilde{\mathcal{C}}^{\prime}$ is given by

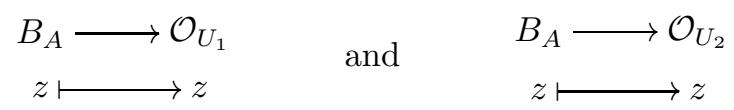

i.e. it is the one induced by base change from the morphism $\tilde{f}_{\text {vers }}$. Since furthermore we have $\varphi \circ \tilde{s}=\tilde{s}$, which is also the morphism induced by base change from $\tilde{s}_{\text {vers }}$, this proves the lemma.

Lemma 6.4. With the notation of Lemma 6.3, let $N:=\tilde{f}_{*}\left(\mathcal{O}_{\tilde{\mathcal{C}}^{\prime}}\left(\tilde{s}^{\prime}\right)\right) \otimes \mathcal{O}_{\tilde{\mathcal{C}}}(-\tilde{s})$ and denote by $\tilde{\mathcal{C}}_{0}^{\prime}, \tilde{s}_{0}^{\prime}$ etc. the objects induced by $\tilde{\mathcal{C}}^{\prime}, \tilde{s}^{\prime}$ etc., via base change $\operatorname{Spec}(k) \rightarrow S$. Then there exist canonical isomorphisms

$$
N \otimes_{\mathcal{O}_{\tilde{\mathcal{C}}}} Q_{A} \stackrel{\sim}{\rightarrow} Q_{A} \quad \text { and } \quad N \otimes_{A} k \stackrel{\sim}{\rightarrow} \mathfrak{m}_{\tilde{\mathcal{C}}_{0}, \tilde{s}_{0}} \oplus\left(\left(\mathfrak{m}_{\tilde{\mathcal{C}}_{0}^{\prime}, \tilde{s}_{0}^{\prime}} / \mathfrak{m}_{\tilde{\mathcal{C}}_{0}^{\prime}, \tilde{s}_{0}^{\prime}}^{2}\right)^{\vee} \otimes \mathfrak{m}_{\tilde{\mathcal{C}}_{0}, \tilde{s}_{0}} / \mathfrak{m}_{\tilde{\mathcal{C}}_{0}, \tilde{s}_{0}}^{2}\right)
$$

where $Q_{A}:=\mathcal{O}_{\tilde{\mathcal{C}} \backslash\{\tilde{s}\}}=A[[z]][1 / z]$, such that the following diagram commutes:

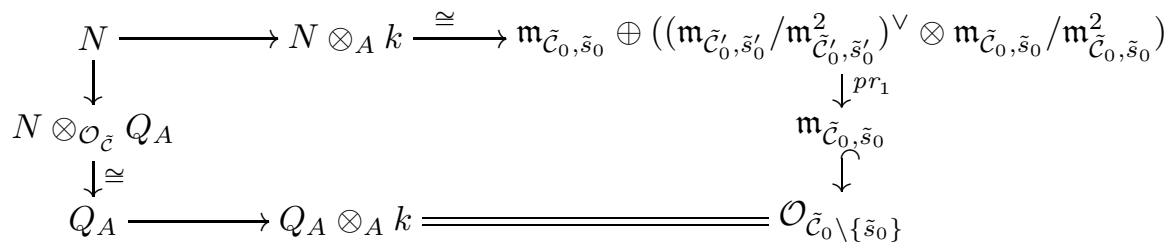


Furthermore, there is an element $a \in \mathfrak{m}_{A}$ and an exact sequence of $\mathcal{O}_{\tilde{\mathcal{C}}}$-modules

$$
\begin{aligned}
0 \longrightarrow \mathcal{O}_{\tilde{\mathcal{C}}} \longrightarrow \mathcal{O}_{\tilde{\mathcal{C}}} \oplus \mathcal{O}_{\tilde{\mathcal{C}}} \longrightarrow N \longrightarrow 0 \\
1 \longmapsto(a,-z)
\end{aligned}
$$

which by tensoring with $Q_{A}$ and $k$ induces the exact sequences

$$
\begin{aligned}
0 \longrightarrow Q_{A} \longrightarrow Q_{A} \oplus Q_{A} \longrightarrow Q_{A} \longrightarrow 0 \\
(1,0) \\
(0,1) \longmapsto z
\end{aligned}
$$

and

$$
\begin{gathered}
0 \longrightarrow \mathcal{O}_{\tilde{\mathcal{C}}_{0} \longrightarrow} \longrightarrow \mathcal{O}_{\tilde{\mathcal{C}}_{0}} \oplus \mathcal{O}_{\tilde{\mathcal{C}}_{0}} \longrightarrow \mathfrak{m}_{\tilde{\mathcal{C}}_{0}, \tilde{s}_{0}} \oplus\left(\left(\mathfrak{m}_{\tilde{\mathcal{C}}_{0}^{\prime}, \tilde{s}_{0}^{\prime}} / \mathfrak{m}_{\tilde{\mathcal{C}}_{0}^{\prime}, \tilde{s}_{0}^{\prime}}^{2}\right)^{\vee} \otimes \mathfrak{m}_{\tilde{\mathcal{C}}_{0}, \tilde{s}_{0}} / \mathfrak{m}_{\tilde{\mathcal{C}}_{0}, \tilde{s}_{0}}^{2}\right) \longrightarrow(z, 0) \\
(1,0) \longmapsto(0, e) \\
(0,1) \longmapsto
\end{gathered}
$$

where $0 \neq e \in\left(\mathfrak{m}_{\tilde{\mathcal{C}}_{0}^{\prime}, \tilde{s}_{0}^{\prime}} / \mathfrak{m}_{\tilde{\mathcal{C}}_{0}^{\prime}, \tilde{s}_{0}^{\prime}}^{2}\right)^{\vee} \otimes \mathfrak{m}_{\tilde{\mathcal{C}}_{0}, \tilde{s}_{0}} / \mathfrak{m}_{\tilde{\mathcal{C}}_{0}, \tilde{s}_{0}}^{2}$.

Proof. Since both $\tilde{f}_{*} \mathcal{O}_{\tilde{\mathcal{C}}^{\prime}}\left(\tilde{s}^{\prime}\right)$ and $\mathcal{O}_{\tilde{\mathcal{C}}}(-\tilde{s})$ are canonically trivialized over $\tilde{\mathcal{C}} \backslash \tilde{s}$, the isomorphism $N \otimes_{\mathcal{O}_{\tilde{\mathcal{C}}}} Q_{A} \stackrel{\sim}{\rightarrow} Q_{A}$ is clear. By $\left[\mathrm{Kn}\right.$, Cor. 1.5, we have $N \otimes_{A} k=$ $\left(f_{0}\right)_{*}\left(\mathcal{M}_{0}^{\vee}\right)$. Let $R$ denote the component isomorphic to $\mathbb{P}_{k}^{1}$ of $\tilde{\mathcal{C}}_{0}^{\prime}$ and by abuse of notation let $\tilde{\mathcal{C}}_{0}$ also denote the component of $\tilde{\mathcal{C}}_{0}^{\prime}$, which is mapped isomorphically to $\tilde{\mathcal{C}}_{0}$ by $\tilde{f}_{0}$. Then we have, similar to the proof of 6.1 ,

$$
\begin{aligned}
\left(f_{0}\right)_{*}\left(\mathcal{M}_{0}^{\vee}\right) & =\left.\mathcal{M}_{0}^{\vee}\right|_{\tilde{\mathcal{C}}_{0}} \times{ }_{\mathcal{M}_{0}^{\vee}\left[\tilde{s}_{0}\right]} H^{0}\left(R,\left.\mathcal{M}_{0}^{\vee}\right|_{R}\right) \\
& =\left.\mathcal{M}_{0}^{\vee}\right|_{\tilde{\mathcal{C}}_{0}} \oplus \operatorname{ker}\left(H^{0}\left(R,\left.\mathcal{M}_{0}^{\vee}\right|_{R}\right) \rightarrow \mathcal{M}_{0}^{\vee}\left[\tilde{s}_{0}\right]\right) \\
& =\mathfrak{m}_{\tilde{\mathcal{C}}_{0}, \tilde{s}_{0}} \oplus\left(\left(\mathfrak{m}_{\tilde{\mathcal{C}}_{0}^{\prime}, \tilde{s}_{0}^{\prime}} / \mathfrak{m}_{\tilde{\mathcal{C}}_{0}^{\prime}, \tilde{s}_{0}^{\prime}}^{2}\right)^{\vee} \otimes \mathfrak{m}_{\tilde{\mathcal{C}}_{0}, \tilde{s}_{0}} / \mathfrak{m}_{\tilde{\mathcal{C}}_{0}, \tilde{s}_{0}}^{2}\right) .
\end{aligned}
$$

The commutativity of diagram $(*)$ comes from the commutativity of the diagram

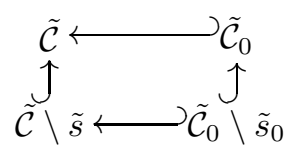

Let $A_{\text {vers }}:=k[[t]]$ and let $S \rightarrow \operatorname{Spec}\left(A_{\text {vers }}\right)$ be a morphism such that $\tilde{\mathcal{C}}_{0}^{\prime} \cong$ $\tilde{\mathcal{C}}_{\text {vers }}^{\prime} \times_{\text {Spec }\left(A_{\text {vers }}\right)} S$. Let $\left.\mathcal{M}_{\text {vers }}^{\vee}:=\mathcal{O}_{\tilde{\mathcal{C}}_{\text {vers }}^{\prime}}\left(\tilde{s}_{\text {vers }}^{\prime}\right)\right) \otimes\left(f_{\text {vers }}\right)^{*}\left(\mathcal{O}_{\tilde{\mathcal{C}}_{\text {vers }}}\left(-\tilde{s}_{\text {vers }}\right)\right)$ and $N_{\text {vers }}$ $:=\left(f_{\text {vers }}\right)_{*} \mathcal{M}_{\text {vers }}^{\vee}$. By $\left[\mathrm{Kn}\right.$, Cor. 1.5 , we have $N=N_{\text {vers }} \otimes_{A_{\text {vers }}} A$. For the existence of the exact sequence $0 \rightarrow \mathcal{O}_{\tilde{\mathcal{C}}} \rightarrow \mathcal{O}_{\tilde{\mathcal{C}}} \oplus \mathcal{O}_{\tilde{\mathcal{C}}} \rightarrow N \rightarrow 0$ it suffices therefore to produce an exact sequence

$$
\begin{gathered}
0 \longrightarrow B_{A_{\text {vers }}} \longrightarrow B_{A_{\text {vers }}} \oplus B_{A_{\text {vers }}} \longrightarrow N_{\text {vers }} \longrightarrow 0 \\
1 \longmapsto(t,-z)
\end{gathered}
$$

where $B_{A_{\text {vers }}}:=A_{\text {vers }}[[z]]=k[[t]][[z]]$. Observe that we have $\mathcal{M}_{\text {vers }}^{\vee}=\mathcal{O}_{\tilde{\mathcal{C}}_{\text {vers }}^{\prime}}(-R)$, where $R$ is the exceptional divisor of the blowing-up morphism $f_{\text {vers. }}$. We have an open affine covering $\tilde{\mathcal{C}}_{\text {vers }}^{\prime}=U_{1} \cup U_{2}$, where $U_{1}=\operatorname{Spec} B_{A_{\text {vers }}}[t / z], U_{2}=$ $\operatorname{Spec} B_{A_{\text {vers }}}[z / t]$ and $U_{1} \cap U_{2}=\operatorname{Spec} B_{A_{\text {vers }}}[t / z, z / t]$. Over $U_{1}$ and $U_{2}$, the divisor $R$ is given by the equation $z$ and $t$, respectively. Therefore we have

$$
N_{\text {vers }}=\left\{(g, h) \in B_{A_{\text {vers }}}[t / z] \times B_{A_{\text {vers }}}[z / t] \mid z \cdot g=t \cdot h \text { in } B_{A_{\text {vers }}}[t / z, z / t]\right\} .
$$


It is easy to see that the morphism $B_{A_{\text {vers }}} \oplus B_{A_{\text {vers }}} \rightarrow N_{\text {vers }}$ given by $(1,0) \mapsto(1, z / t)$ and $(0,1) \mapsto(t / z, 1)$ is surjective whith kernel $B_{A_{\text {vers }}} \cdot(t,-z)$. The compatibility (in the sense of the lemma) of the exact sequence $(\dagger)$ with the canonical isomorphisms $\left.N_{\text {vers }}\right|_{\left(\tilde{\mathcal{C}}_{\text {vers }} \backslash s_{\text {vers })}\right.} \stackrel{\sim}{\rightarrow} B_{A_{\text {vers }}}[1 / z]$ and $\left.N_{\text {vers }}\right|_{\tilde{\mathcal{C}}_{0}} \stackrel{\sim}{\rightarrow} \mathfrak{m}_{\tilde{\mathcal{C}}_{0}, \tilde{s}_{0}} \oplus\left(\left(\mathfrak{m}_{\tilde{\mathcal{C}}_{0}^{\prime}, \tilde{s}_{0}^{\prime}} / \mathfrak{m}_{\tilde{\mathcal{C}}_{0}^{\prime}, \tilde{s}_{0}^{\prime}}^{2}\right)^{\vee} \otimes\right.$ $\left.\mathfrak{m}_{\tilde{\mathcal{C}}_{0}, \tilde{s}_{0}} / \mathfrak{m}_{\tilde{\mathcal{C}}_{0}, \tilde{s}_{0}}^{2}\right)$ follows by inspection.

Lemma 6.5. In the situation of 5.7 let $S=\operatorname{Spec}(A)$ for a local artinian $k$-algebra $A$ with residue field $k$ and let $\mathcal{C}_{0}^{\prime}:=\mathcal{C}^{\prime} \otimes_{A} k$. Then the restriction map

$$
H^{0}\left(\mathcal{C}^{\prime}, \mathcal{M}^{\vee}\right) \rightarrow H^{0}\left(\mathcal{C}_{0}^{\prime},\left.\mathcal{M}^{\vee}\right|_{\mathcal{C}_{0}^{\prime}}\right)
$$

is surjective.

Proof. Let $\mathcal{N}$ be the $\mathcal{O}_{\mathcal{C}}$-module $f_{*}\left(\mathcal{M}^{\vee}\right)$. By $\underline{\mathrm{Kn}}$, Cor. 1.5, the formation of $\mathcal{N}$ commutes with base change $S^{\prime} \rightarrow S$. Therefore it suffices to show the surjectivity of the restriction morphism

$$
H^{0}(\mathcal{C}, \mathcal{N}) \rightarrow H^{0}\left(\mathcal{C}_{0}, \mathcal{N}_{0}\right)
$$

where $\mathcal{C}_{0}:=\mathcal{C} \otimes_{A} k$ and $\mathcal{N}_{0}:=\left.\mathcal{N}\right|_{\mathcal{C}_{0}}$. Let $\tilde{\mathcal{C}}$ be the completion of $\mathcal{C}$ along the section $s$. Since $\pi$ is smooth in the neighbourhood of $s$, we may identify $\tilde{\mathcal{C}}$ with $\operatorname{Spec}(A[[z]])$ such that $z$ corresponds to a generator of the $\mathcal{O}_{\mathcal{C}}$-Ideal defining $s$. Let $\tilde{\mathcal{C}}^{\prime}:=\mathcal{C}^{\prime} \times_{\mathcal{C}} \tilde{\mathcal{C}}$ and denote by $\tilde{f}$ the projection $\tilde{\mathcal{C}}^{\prime} \rightarrow \tilde{\mathcal{C}}$ onto the second factor. Then we have a canonical isomorphism $N:=\tilde{f}_{*}\left(\mathcal{O}_{\tilde{\mathcal{C}}^{\prime}}\left(\tilde{s}^{\prime}\right)\right) \otimes \mathcal{O}_{\tilde{\mathcal{C}}}(-\tilde{s}) \stackrel{\sim}{\rightarrow} \mathcal{N} \otimes_{\mathcal{O}_{\mathcal{C}}} \mathcal{O}_{\tilde{\mathcal{C}}}$. Let $Q_{A}$ be the localization of $\mathcal{O}_{\tilde{\mathcal{C}}}$ with respect to $z$. Since $\mathcal{N}$ is naturally trivialized on the complement of $s$, we have canonical isomorphisms $\mathcal{N} \otimes_{\mathcal{O}_{\mathcal{C}}} Q_{A} \stackrel{\sim}{\rightarrow} Q_{A}$ and $N \otimes_{\mathcal{O}_{\tilde{\mathcal{C}}}} Q_{A} \stackrel{\sim}{\rightarrow} Q_{A}$. By $[\mathrm{B}-\mathrm{L}$ ] the canonical morphism

$$
H^{0}(\mathcal{C}, \mathcal{N}) \rightarrow N \times{ }_{Q_{A}} H^{0}(\mathcal{C} \backslash\{s\}, \mathcal{N})
$$

is an isomorphism. Likewise, we have a canonical isomorphism

$$
H^{0}\left(\mathcal{C}_{0}, \mathcal{N}_{0}\right) \rightarrow N_{0} \times_{Q_{k}} H^{0}\left(\mathcal{C}_{0} \backslash\{s\}, \mathcal{N}_{0}\right)
$$

where $N_{0}:=N \otimes_{A} k$ and $Q_{k}:=Q_{A} \otimes_{A} k$. By the first part of Lemma 6.4 we have

$$
\begin{aligned}
N_{0} \times Q_{Q_{k}} H^{0}\left(\mathcal{C}_{0} \backslash\{s\}, \mathcal{N}_{0}\right) & =H^{0}\left(\mathcal{C}_{0}, \mathcal{O}_{\mathcal{C}_{0}}\left(-s_{0}\right)\right) \oplus\left(\left(\mathfrak{m}_{\tilde{\mathcal{C}}_{0}^{\prime}, \tilde{s}_{0}^{\prime}} / \mathfrak{m}_{\tilde{\mathcal{C}}_{0}^{\prime}, \tilde{s}_{0}^{\prime}}^{2}\right)^{\vee} \otimes \mathfrak{m}_{\tilde{\mathcal{C}}_{0}, \tilde{s}_{0}} / \mathfrak{m}_{\tilde{\mathcal{C}}_{0}, \tilde{s}_{0}}^{2}\right) \\
& =\left(\mathfrak{m}_{\tilde{\mathcal{C}}_{0}^{\prime}, \tilde{s}_{0}^{\prime}} / \mathfrak{m}_{\tilde{\mathcal{C}}_{0}^{\prime}, \tilde{s}_{0}^{\prime}}^{2}\right)^{\vee} \otimes \mathfrak{m}_{\tilde{\mathcal{C}}_{0}, \tilde{s}_{0}} / \mathfrak{m}_{\tilde{\mathcal{C}}_{0}, \tilde{s}_{0}}^{2} .
\end{aligned}
$$

Let $\nu \in N$ be the image of $(0,1) \in \mathcal{O}_{\tilde{\mathcal{C}}} \oplus \mathcal{O}_{\tilde{\mathcal{C}}}$ by the surjection $\mathcal{O}_{\tilde{\mathcal{C}}} \oplus \mathcal{O}_{\tilde{\mathcal{C}}} \rightarrow N$ from Lemma 6.4 By the second part of Lemma 6.4 the pair $(\nu, a)$ is an element of $N \times{ }_{Q_{A}} H^{0}(\mathcal{C} \backslash\{s\}, \mathcal{N})$ and its image under the restriction morphism

$N \times{ }_{Q_{A}} H^{0}(\mathcal{C} \backslash\{s\}, \mathcal{N}) \rightarrow N_{0} \times Q_{Q_{k}} H^{0}\left(\mathcal{C}_{0} \backslash\{s\}, \mathcal{N}_{0}\right)=\left(\mathfrak{m}_{\tilde{\mathcal{C}}_{0}^{\prime}, \tilde{s}_{0}^{\prime}} / \mathfrak{m}_{\tilde{\mathcal{C}}_{0}^{\prime}, \tilde{s}_{0}^{\prime}}^{2} \vee^{\vee} \otimes \mathfrak{m}_{\tilde{\mathcal{C}}_{0}, \tilde{s}_{0}} / \mathfrak{m}_{\tilde{\mathcal{C}}_{0}, \tilde{s}_{0}}^{2}\right.$ is $e \neq 0$.

Lemma 6.6. Let $S$ be a locally noetherian scheme, and let $\pi: \mathcal{C} \rightarrow S$ be a finitely presented flat morphism whose geometric fibres are connected of dimension one. Let $f: \mathcal{C}^{\prime} \rightarrow \mathcal{C}$ be a proper morphism such that $\pi^{\prime}:=\pi \circ f$ is flat. Let $s^{\prime}: S \rightarrow \mathcal{C}^{\prime}$ be a section of $\pi^{\prime}$ and let $s:=f \circ s^{\prime}$. Assume that the restriction of $f$ to $\mathcal{C}^{\prime} \backslash s^{\prime}(S)$ is an isomorphism onto $\mathcal{C} \backslash s(S)$. Let $z \in S$ be a point. Denote by $\mathcal{C}_{z}$ and $\mathcal{C}_{z}^{\prime}$ the fibres of $\pi$ and of $\pi^{\prime}$ over $z$ and by $\pi_{z}, \pi_{z}^{\prime}, f_{z}, s_{z}, s_{z}^{\prime}$ the the restriction of $\pi, \pi^{\prime}, f, s, s^{\prime}$ on the fibres. Assume that $\left(\mathcal{C}_{z}^{\prime}, f_{z}, \pi_{z}^{\prime}, s_{z}^{\prime}\right)$ is a simple modification of $\left(\mathcal{C}_{z}, \pi_{z}, s_{z}\right)$. Then there is an open neighbourhood $U \subseteq S$ of $z$, such that $\left.\left(\mathcal{C}^{\prime}, f, \pi^{\prime}, s^{\prime}\right)\right|_{U}$ is a simple modification of $\left.(\mathcal{C}, \pi, s)\right|_{U}$. If we assume in addition that $H^{0}\left(\mathcal{C}_{z}, \mathcal{O}_{\mathcal{C}_{z}}\right)=\kappa(z)$ and 
$H^{0}\left(\mathcal{C}_{z}, \mathcal{O}_{\mathcal{C}_{z}}(-s(z))\right)=(0)$, then we can choose $U$ such that the analogous property holds for all $z^{\prime} \in U$.

Proof. Admitting the first part of the lemma, the last assertion in the lemma follows immediately by [EGA III], (7.8.7), (7.8.8) and (7.7.5)I. By assumption, the $s(z)$ and $s^{\prime}(z)$ are smooth points in their fibre. Therefore there exists an open neighbourhood $V \subseteq \mathcal{C}$ of $s(z)$ and $V^{\prime} \subseteq \mathcal{C}^{\prime}$ of $s^{\prime}(z)$ such that the restriction of $\pi$ to $V$ and the restriction of $\pi^{\prime}$ to $V^{\prime}$ is smooth (EGA IV], (12.1.7)(iv)). Replacing $S$ by $s^{-1}(V) \cap$ $\left(s^{\prime}\right)^{-1}\left(V^{\prime}\right)$, we may assume that $s$ and $s^{\prime}$ meet $\mathcal{C}$ and $\mathcal{C}^{\prime}$ in the smooth locus of $\pi$ and $\pi^{\prime}$. If $f_{z}$ is an isomorphism, then by EGA IV], (17.8.3), and [EGA III], (4.4.5), there is a neighbourhood $W \subseteq \mathcal{C}$ of $s(z)$ such that the restriction of $f$ to $W^{\prime}:=f^{-1}(W)$ is a finite etale morphism $W^{\prime} \rightarrow W$. Since its degree over $s(z)$ is one, it is in fact an isomorphism. It is clear that in this case $U:=s^{-1}(W)$ has the required property. Now assume that $f_{z}$ is not an isomorphism. Let $\widehat{S}$ be the completion of $S$ at $z$ and let $\widehat{\mathcal{C}}$ (resp. $\widehat{\mathcal{C}}^{\prime}$ ) be the completion of $\mathcal{C} \times{ }_{S} \widehat{S}$ (resp. of $\left.\mathcal{C}^{\prime} \times{ }_{S} \widehat{S}\right)$ along the subscheme $s(S) \times{ }_{S} \widehat{S}\left(\right.$ resp. $\left.s^{\prime}(S) \times{ }_{S} \widehat{S}\right)$. Denote by $\widehat{\pi}, \widehat{\pi}^{\prime}$, $\widehat{f}, \widehat{s}, \widehat{s}^{\prime}$ the morphisms between $\widehat{S}, \widehat{\mathcal{C}}$ and $\widehat{\mathcal{C}}^{\prime}$ induced by $\pi, \pi^{\prime}, f, s, s^{\prime}$. From Lemma 6.2 it follows that $\left(\widehat{\mathcal{C}^{\prime}}, \widehat{f}, \widehat{\pi}^{\prime}, \widehat{s}^{\prime}\right)$ is a simple modification of $(\widehat{\mathcal{C}}, \widehat{\pi}, \widehat{s})$. Since $\widehat{S} \rightarrow \operatorname{Spec}\left(\mathcal{O}_{S, z}\right)$ is surjective (cf. [Ma], §8), it follows that $\left.\left(\mathcal{C}^{\prime}, f, \pi^{\prime}, s^{\prime}\right)\right|_{\operatorname{Spec}\left(\mathcal{O}_{S, z}\right)}$ is a simple modification of $\left.(\mathcal{C}, \pi, s)\right|_{\operatorname{Spec}\left(\mathcal{O}_{S, z}\right)}$. Thus it suffices to show that the set $T$ of all $z^{\prime} \in S$ such that $\left(\mathcal{C}_{z^{\prime}}^{\prime}, f_{z^{\prime}}, \pi_{z^{\prime}}^{\prime}, s_{z^{\prime}}^{\prime}\right)$ is a simple modification of $\left(\mathcal{C}_{z^{\prime}}, \pi_{z^{\prime}}, s_{z^{\prime}}\right)$ is a locally constructible subset of $S$. But this is a consequence of the following characterization of $T$ : A point $z^{\prime} \in S$ is contained in $T$ if and only if (i) $f^{-1}\left(s\left(z^{\prime}\right)\right)$ is irreducible, smooth and of dimension $\leq 1$. (ii) $H^{1}\left(f^{-1}\left(s\left(z^{\prime}\right)\right), \mathcal{O}\right)=0$. (iii) $\left(\pi^{\prime}\right)^{-1}\left(z^{\prime}\right) \cap f^{-1}(V)$ is connected and is either smooth or contains precisely one ordinary double point.

Lemma 6.7. Let $S$ be an affine scheme, let $\pi: \mathcal{C} \rightarrow S$ be a proper finitely presented flat morphism whose geometric fibres are connected of dimension one and let $s: S \rightarrow \mathcal{C}$ be a section of $\pi$ whose image consists of points where $\pi$ is smooth. Let $\left(\mathcal{C}^{\prime}, f, \pi^{\prime}, s^{\prime}\right)$ be a simple modification of $(\mathcal{C}, \pi, s)$. Then there is a noetherian scheme $S_{0}$, a proper flat morphism $\pi_{0}: \mathcal{C}_{0} \rightarrow S_{0}$ with connected geometric fibres of dimension one, a section $s_{0}$ of $\pi_{0}$ meeting $\mathcal{C}_{0}$ in the smooth locus of $\pi_{0}$, a simple modification $\left(\mathcal{C}_{0}^{\prime}, f_{0}, \pi_{0}^{\prime}, s_{0}^{\prime}\right)$ of $\left(\mathcal{C}_{0}, \pi_{0}, s_{0}\right)$ and a morphism $S \rightarrow S_{0}$ such that the data $\left(\mathcal{C}^{\prime}, f, \pi^{\prime}, s^{\prime}, \mathcal{C}, \pi, s\right)$ are induced by pull back from the data $\left(\mathcal{C}_{0}^{\prime}, f_{0}, \pi_{0}^{\prime}, s_{0}^{\prime}, \mathcal{C}_{0}, \pi_{0}, s_{0}\right)$. If we assume in addition that $H^{0}\left(\mathcal{C}_{z}, \mathcal{O}_{\mathcal{C}_{z}}\right)=\kappa(z)$ and $H^{0}\left(\mathcal{C}_{z}, \mathcal{O}_{\mathcal{C}_{z}}(-s(z))\right)=(0)$ for all $z \in S$, then we can choose $\left(\mathcal{C}_{0}, \pi_{0}, s_{0}\right)$ such that the analogous property holds for all $z_{0} \in S_{0}$.

Proof. Let $A$ be the coordinate ring of $S$ and let $\left(A_{\lambda}\right)_{\lambda}$ be the inductive system of all noetherian subrings of $A$. Let $S_{\lambda}:=\operatorname{Spec}\left(A_{\lambda}\right)$ for all $\lambda$. Using the results from [EGA IV] cited in the proof of 3.15 one shows that there exists an index $\lambda$, a proper flat morphism $\pi_{\lambda}: \mathcal{C}_{\lambda} \rightarrow S_{\lambda}$, a proper morphism $f_{\lambda}: \mathcal{C}_{\lambda}^{\prime} \rightarrow \mathcal{C}_{\lambda}$ such that the composition $\pi_{\lambda}^{\prime}:=\pi_{\lambda} \circ f \lambda$ is flat, and a morphism $s_{\lambda}^{\prime}: S_{\lambda} \rightarrow \mathcal{C}_{\lambda}^{\prime}$ such that the data $\left(\mathcal{C}^{\prime}, f, \pi^{\prime}, s^{\prime}, \mathcal{C}, \pi, s\right)$ are induced from the data $\left(\mathcal{C}_{\lambda}^{\prime}, f_{\lambda}, \pi_{\lambda}^{\prime}, s_{\lambda}^{\prime}, \mathcal{C}_{\lambda}, \pi_{\lambda}, s_{\lambda}\right)$, where $s_{\lambda}:=f \circ s_{\lambda}^{\prime}$. By [EGA IV], (8.8.2)(i) and (8.8.2.4), we may assume that $s_{\lambda}^{\prime}$ is a section of $\pi_{\lambda}^{\prime}$ and that the restriction of $f_{\lambda}$ to the open subset $f_{\lambda}^{-1}\left(\mathcal{C}_{\lambda} \backslash s_{\lambda}\left(S_{\lambda}\right)\right)$ of $\mathcal{C}_{\lambda}^{\prime}$ is an isomorphism onto $\mathcal{C}_{\lambda} \backslash s_{\lambda}\left(S_{\lambda}\right)$. To conclude, we now make use of 6.6 similarly as we employed 3.14 in the proof of Lemma 3.15 
Proof of Lemma 5.7. Consider first the case where $S$ is locally noetherian. Then both $\pi_{*}^{\prime} \mathcal{M}^{\vee}$ and $\left(s^{\prime}\right)^{*} \mathcal{M}^{\vee}$ are coherent $S$-modules and $\left(s^{\prime}\right)^{*} \mathcal{M}^{\vee}$ is locally free, so Lemma 5.7 follows easily from Lemma 6.1. if we can show that the formation of $\pi_{*}^{\prime} \mathcal{M}^{\vee}$ commutes with base change by a morphism from any scheme to $S$. But this base change property follows from Lemma 6.5] and [EGA III], 7.7.5 and 7.7.10. In the general case we may assume $S$ to be affine. By Lemma 6.7 there exists a morphism from $S$ to a locally noetherian scheme $S_{0}$ such that the given data $\left(\mathcal{C}^{\prime}, f, \pi^{\prime}, s^{\prime}, \mathcal{C}, \pi, s\right)$ are induced by pull back from data $\left(\mathcal{C}_{0}^{\prime}, f_{0}, \pi_{0}^{\prime}, s_{0}^{\prime}, \mathcal{C}_{0}, \pi_{0}, s_{0}\right)$ defined over $S_{0}$. Let $\mathcal{M}_{0}^{\vee}:=\mathcal{O}_{\mathcal{C}_{0}^{\prime}}\left(s_{0}^{\prime}\right) \otimes_{\mathcal{O}_{\mathcal{C}_{0}^{\prime}}} f_{0}^{*} \mathcal{O}_{\mathcal{C}_{0}}\left(-s_{0}\right)$. By the first case, we have $\left(\pi_{0}^{\prime}\right)_{*} \mathcal{M}_{0}^{\vee} \stackrel{\sim}{\rightarrow}\left(s_{0}^{\prime}\right)^{*} \mathcal{M}_{0}^{\vee}$. Since furthermore our morphism $\pi_{*}^{\prime} \mathcal{M}^{\vee} \rightarrow\left(s^{\prime}\right)^{*} \mathcal{M}^{\vee}$ is induced from this morphism by base change, we are done.

\section{Admissible BUNDLES ON SIMPLE MODIFICATIONS AND BF-MORPHISMS}

Throughout this section, $S$ will be a locally Noetherean scheme, $\pi: \mathcal{C} \rightarrow S$ a proper and flat morphism with connected geometric fibers of dimension one such that $\pi_{*} \mathcal{O}_{\mathcal{C}}=\mathcal{O}_{S}$, and $s: S \rightarrow \mathcal{C}$ will be a section of $\pi$ whose image consists of smooth points of $\pi$. Furthermore we fix an $\mathcal{O}_{\mathcal{C}}$-module $\mathcal{E}$ and an $\mathcal{O}_{S}$-module $E^{\prime}$, both locally free of rank $n$. Let $E:=s^{*} \mathcal{E}$.

Definition 7.1. Let $\left(\mathcal{C}^{\prime}, f, \pi^{\prime}, s^{\prime}\right)$ be a simple modification of $(\mathcal{C}, \pi, s)$ (cf. 5.1) and

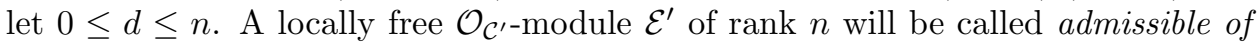
degree $d$ for $\left(\mathcal{C}^{\prime}, f, \pi^{\prime}, s^{\prime}\right)$, if for any point $x \in \mathcal{C}$ with $f^{-1}(x) \cong \mathbb{P}_{\kappa(x)}^{1}$ we have

$$
\left.\mathcal{E}^{\prime}\right|_{f^{-1}(x)} \cong \bigoplus^{d} \mathcal{O}_{\mathbb{P}_{\kappa(x)}^{1}}(1) \oplus \bigoplus^{n-d} \mathcal{O}_{\mathbb{P}_{\kappa(x)}^{1}}
$$

Construction 7.2. Let $\left(\mathcal{C}^{\prime}, f, \pi^{\prime}, s^{\prime}\right)$ be a simple modification of $(\mathcal{C}, \pi, s)$ and let $\mathcal{E}^{\prime}$ be an admissible $\mathcal{O}_{\mathcal{C}^{\prime}}$-module of degree $d$ together with isomorphisms

$$
\left(f_{*} \mathcal{E}^{\prime}\left(-s^{\prime}\right)\right)(s) \stackrel{\sim}{\rightarrow} \mathcal{E} \quad \text { and } \quad\left(s^{\prime}\right)^{*} \mathcal{E}^{\prime} \stackrel{\sim}{\rightarrow} E^{\prime}
$$

Since $R^{1} f_{*} \mathcal{E}^{\prime}\left(-s^{\prime}\right)=0$ by $[\mathrm{Kn}, 1.5$, we have the commutative diagram with exact rows:

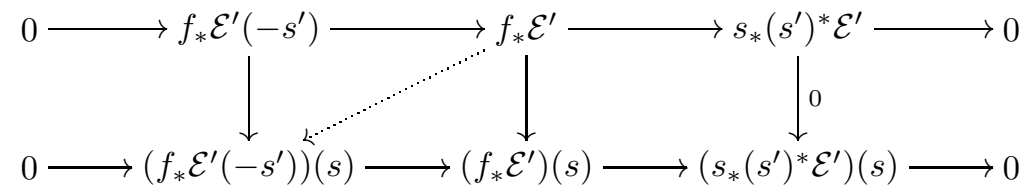

The vanishing of the right vertical arrow implies that the middle vertical arrow factorizes as indicated by the dotted arrow. Composing this arrow with the isomorphism $\left(f_{*} \mathcal{E}^{\prime}\left(-s^{\prime}\right)\right)(s) \stackrel{\sim}{\rightarrow} \mathcal{E}$, we obtain a morphism $f_{*} \mathcal{E}^{\prime} \rightarrow \mathcal{E}$. Now consider the following diagram with exact rows:

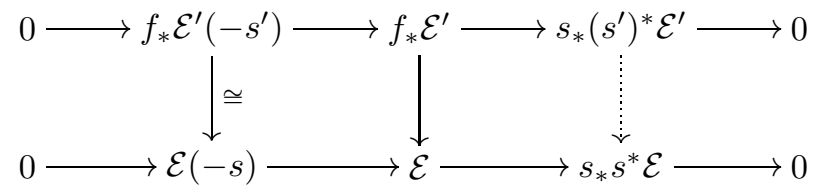

By the commutativity of the left square we obtain a morphism as indicated by the dotted arrow. This induces a morphism $E^{\prime} \rightarrow E$. 
Let $\mathcal{M}:=\mathcal{O}_{\mathcal{C}^{\prime}}\left(-s^{\prime}\right) \otimes f^{*} \mathcal{O}_{\mathcal{C}}(s)$. By the projection formula we have $f_{*}\left(\mathcal{M} \otimes \mathcal{E}^{\prime}\right)=$ $\left(f_{*} \mathcal{E}^{\prime}\left(-s^{\prime}\right)\right)(s)$. Thus we obtain a morphism

$$
E=s^{*} \mathcal{E} \stackrel{\sim}{\rightarrow} s^{*} f_{*}\left(\mathcal{M} \otimes \mathcal{E}^{\prime}\right)=\left(s^{\prime}\right)^{*} f^{*} f_{*}\left(\mathcal{M} \otimes \mathcal{E}^{\prime}\right) \rightarrow\left(s^{\prime}\right)^{*}\left(\mathcal{M} \otimes \mathcal{E}^{\prime}\right) \stackrel{\sim}{\rightarrow} M \otimes E^{\prime},
$$

where $(M, \mu)$ is the invertible $\mathcal{O}_{S}$-module together with a global section associated to the simple modification $\left(\mathcal{C}^{\prime}, f, \pi^{\prime}, s^{\prime}\right)$ (cf. Proposition 5.3).

Remark 7.3. In the case $S=\operatorname{Spec}(K)$ for a field $K$, the curve $\mathcal{C}$ can be identified with a closed subscheme of $\mathcal{C}^{\prime}$ and Construction 7.2 can be specified more concretely as follows. Assume that $f$ is not an isomorphism and let $R:=f^{-1}(s) \cong \mathbb{P}_{K}^{1}$. Then $M=\left(\mathfrak{m} / \mathfrak{m}^{2}\right)^{\vee} \otimes\left(\mathfrak{m}^{\prime} /\left(\mathfrak{m}^{\prime}\right)^{2}\right)$ and $\mu=0$, where $\mathfrak{m}$ (resp. $\left.\mathfrak{m}^{\prime}\right)$ is the maximal ideal belonging to the point $s \in \mathcal{C}$ (resp. to the point $s^{\prime} \in \mathcal{C}^{\prime}$ ). Observe that we have

$$
\mathcal{O}_{\mathcal{C}, s} \otimes f_{*} \mathcal{E}^{\prime}\left(-s^{\prime}\right)=\left(\left.\mathcal{E}^{\prime}\right|_{\mathcal{C}}\right)_{s} \times_{\mathcal{E}^{\prime}[s]} H^{0}\left(R,\left.\mathcal{E}^{\prime}\left(-s^{\prime}\right)\right|_{R}\right)=\left(\left.\mathcal{E}^{\prime}\right|_{\mathcal{C}}\right)_{s} \times_{\mathcal{E}^{\prime}[s]} \mathcal{F}[s]
$$

where $\left.0 \rightarrow \mathcal{F} \rightarrow \mathcal{E}^{\prime}\right|_{R} \rightarrow \mathcal{G} \rightarrow 0$ is the canonical exact sequence with $\mathcal{F} \cong \bigoplus^{d} \mathcal{O}_{R}(1)$ and $\mathcal{G} \cong \bigoplus^{n-d} \mathcal{O}_{R}$. Therefore we have a canonical exact sequence of $\mathcal{O}_{\mathcal{C}}$-modules:

$$
\left.0 \rightarrow \mathcal{E}(-s) \rightarrow \mathcal{E}^{\prime}\right|_{\mathcal{C}} \rightarrow \mathcal{G}[s] \rightarrow 0
$$

Tensoring this exact sequence with $K=\kappa(s)$ leads to the exact sequence

$$
0 \rightarrow\left(\mathfrak{m} / \mathfrak{m}^{2}\right) \otimes \mathcal{G}[s] \rightarrow\left(\mathfrak{m} / \mathfrak{m}^{2}\right) \otimes E \rightarrow \mathcal{E}^{\prime}[s] \rightarrow \mathcal{G}[s] \rightarrow 0
$$

and the image of $\left(\mathfrak{m} / \mathfrak{m}^{2}\right) \otimes E \rightarrow \mathcal{E}^{\prime}[s]$ is the vector space $\mathcal{F}[s]$ which is canonically isomorphic to $\left(\mathfrak{m}^{\prime} /\left(\mathfrak{m}^{\prime}\right)^{2}\right) \otimes \mathcal{F}\left[s^{\prime}\right]$. Therefore we arrive at the two exact sequences:

$$
\begin{gathered}
0 \longrightarrow \mathcal{F}\left[s^{\prime}\right] \longrightarrow E^{\prime} \longrightarrow \mathcal{G}\left[s^{\prime}\right]=\mathcal{G}[s] \longrightarrow 0 \\
0 \longrightarrow \mathcal{G}[s] \longrightarrow E \longrightarrow \mathcal{F}\left[s^{\prime}\right] \longrightarrow 0
\end{gathered}
$$

The morphisms $E^{\prime} \rightarrow E$ and $E \rightarrow M \otimes E^{\prime}$ from Construction 7.2 are just the compositions $E^{\prime} \rightarrow \mathcal{G}[s] \rightarrow E$ and $E \rightarrow M \otimes \mathcal{F}\left[s^{\prime}\right] \rightarrow M \otimes E^{\prime}$.

Proposition 7.4. Construction 7.2 defines an isomorphism of groupoids:

$$
\left\{\begin{array}{l}
\text { simple modifications } \\
\left(\mathcal{C}^{\prime}, f, \pi^{\prime}, s^{\prime}\right) \text { of }(\mathcal{C}, \pi, s) \\
\text { together with an } \\
\text { admissible } \mathcal{O}_{\mathcal{C}^{\prime}-\text {-module } \mathcal{E}^{\prime} \text { of }} \\
\text { degree } d \text { and isomorphisms } \\
\left(f_{*} \mathcal{E}^{\prime}\left(-s^{\prime}\right)\right)(s) \stackrel{\sim}{\rightarrow} \mathcal{E} \\
\text { and }\left(s^{\prime}\right)^{*} \mathcal{E}^{\prime} \stackrel{\sim}{\rightarrow} E^{\prime}
\end{array}\right\} \stackrel{\sim}{\rightarrow}\left\{\begin{array}{l}
\text { bf-morphisms } \\
\left(M, \mu, E^{\prime} \rightarrow E, M \otimes E^{\prime} \leftarrow E, n-d\right) \\
\text { of rank } n-d \text { from } E^{\prime} \text { to E } \\
\text { in the sense of }[\mathrm{K1}, 3.1
\end{array}\right\}
$$

In the remainder of this section we will prove Proposition 7.4. Let $M$ be an invertible $\mathcal{O}_{S}$-module and $\mu$ a global section of $M$. Let $\left(\mathcal{C}^{\prime}, f, \pi^{\prime}, s^{\prime}\right)$ be the corresponding simple modification of $(\mathcal{C}, \pi, s)$ (cf. Proposition 5.3). Let $\mathcal{E}_{1}$ and $\mathcal{E}_{2}$ be two locally free $\mathcal{O}_{\mathcal{C}}$-modules of rank $d$ and $n-d$ respectively. To these data we associate a locally free $\mathcal{O}_{\mathcal{C}^{\prime}}$-module $\mathcal{E}^{\prime}\left(\mathcal{E}_{1}, \mathcal{E}_{2}\right)$ of rank $n$ as follows:

$$
\mathcal{E}^{\prime}\left(\mathcal{E}_{1}, \mathcal{E}_{2}\right):=\left(\mathcal{M}^{\vee} \otimes f^{*} \mathcal{E}_{1}\right) \oplus f^{*} \mathcal{E}_{2} \quad,
$$

where $\mathcal{M}:=\mathcal{O}_{\mathcal{C}^{\prime}}\left(-s^{\prime}\right) \otimes f^{*} \mathcal{O}_{\mathcal{C}}(s)$. 
Lemma 7.5. Let $\mathcal{E}^{\prime}:=\mathcal{E}^{\prime}\left(\mathcal{E}_{1}, \mathcal{E}_{2}\right)$ and $E_{i}:=s^{*} \mathcal{E}_{i}$ for $i=1,2$. Then $\mathcal{E}^{\prime}$ is an

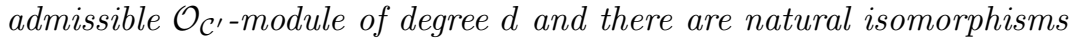

$$
\left(f_{*} \mathcal{E}^{\prime}\left(-s^{\prime}\right)\right)(s) \stackrel{\sim}{\rightarrow} \mathcal{E}_{1} \oplus \mathcal{E}_{2} \quad \text { and } \quad\left(s^{\prime}\right)^{*} \mathcal{E}^{\prime} \stackrel{\sim}{\rightarrow}\left(M^{\vee} \otimes E_{1}\right) \oplus E_{2}
$$

Furthermore, the following diagram commutes:

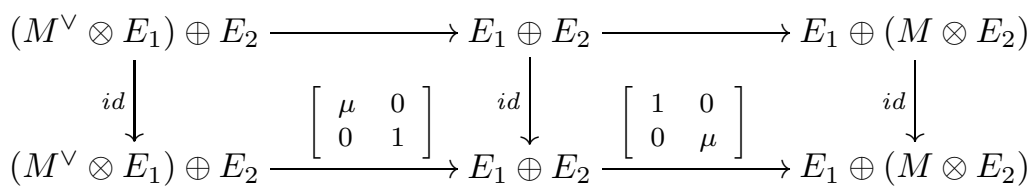

where the upper horizontal arrows are defined as in Construction 7.2 .

Proof. It is clear that $\mathcal{E}^{\prime}$ is admissible. By Lemma 5.9 we have canonical isomorphisms $f_{*} \mathcal{O}_{\mathcal{C}^{\prime}} \stackrel{\sim}{\rightarrow} \mathcal{O}_{\mathcal{C}}$ and $f_{*} \mathcal{M} \stackrel{\sim}{\rightarrow} \mathcal{O}_{\mathcal{C}}$. Therefore we get an isomorphism

$$
\left(f_{*} \mathcal{E}^{\prime}\left(-s^{\prime}\right)\right)(s)=f_{*}\left(\mathcal{M} \otimes \mathcal{E}^{\prime}\right)=\left(f_{*} \mathcal{O}_{\mathcal{C}^{\prime}} \otimes \mathcal{E}_{1}\right) \oplus\left(f_{*} \mathcal{M} \otimes \mathcal{E}_{2}\right) \stackrel{\sim}{\rightarrow} \mathcal{E}_{1} \oplus \mathcal{E}_{2}
$$

The isomorphism $\left(s^{\prime}\right)^{*} \mathcal{E}^{\prime} \stackrel{\sim}{\rightarrow}\left(M^{\vee} \otimes E_{1}\right) \oplus E_{2}$ is the obvious one. For the commutativity of the diagram it suffices to consider the two cases $\left(\mathcal{E}_{1}, \mathcal{E}_{2}\right)=\left(\mathcal{O}_{\mathcal{C}}, 0\right)$ and $\left(\mathcal{E}_{1}, \mathcal{E}_{2}\right)=\left(0, \mathcal{O}_{\mathcal{C}}\right)$. In the first case $\mathcal{E}^{\prime}=\mathcal{M}^{\vee}$. The main point in this case is the observation that the diagram

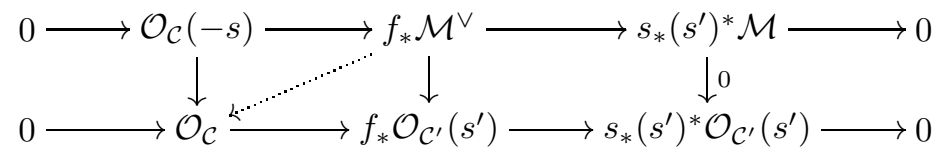

defining $f_{*} \mathcal{M}^{\vee}=f_{*} \mathcal{E}^{\prime} \rightarrow\left(f_{*} \mathcal{E}^{\prime}\left(-s^{\prime}\right)\right)(s)=\mathcal{O}_{\mathcal{C}}$ (cf. Construction 7.2) is obtained by applying $f_{*}$ to the diagram

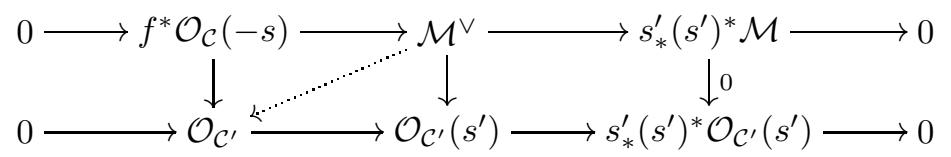

and that the dotted arrow of this last diagram is dual to the dotted arrow in Construction 5.2. If $\left(\mathcal{E}_{1}, \mathcal{E}_{2}\right)=\left(0, \mathcal{O}_{\mathcal{C}}\right)$, then $\mathcal{E}^{\prime}=\mathcal{O}_{\mathcal{C}^{\prime}}$. Here the main point is to show that the composition of the isomorphism $\mathcal{O}_{\mathcal{C}^{\prime}} \stackrel{\sim}{\rightarrow} f^{*} f_{*} \mathcal{M}$ with the adjunction morphism $f^{*} f_{*} \mathcal{M} \rightarrow \mathcal{M}$ coincides with the dotted arrow in Construction [5.2. But this follows from the commutativity of the diagram

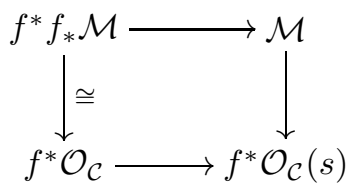

which in turn is easy to see.

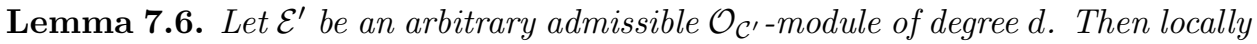
in a neighbourhood of $s(S) \subset \mathcal{C}$ there exist free $\mathcal{O}_{\mathcal{C}}$-modules $\mathcal{E}_{1}$ and $\mathcal{E}_{2}$ of rank $d$ and $n-d$ respectively, such that $\mathcal{E}^{\prime}$ is isomorphic to $\mathcal{E}^{\prime}\left(\mathcal{E}_{1}, \mathcal{E}_{2}\right)$. 
Proof. We may assume that $S=\operatorname{Spec}(A), \mathcal{C}=\operatorname{Spec}(B)$ for a noetherian ring $A$ and smooth $A$-algebra $B$. By Proposition 5.3 we may assume furthermore that $\left(\mathcal{C}^{\prime}, f, \pi^{\prime}, s^{\prime}\right)$ is the simple modification of $(\mathcal{C}, \pi, s)$ associated to a pair $(M, \mu)$ by Construction [5.4, where $M$ is a free $A$-module of rank one. Let $A_{0}:=$ $\operatorname{coker}\left(\mu: \mathcal{M}^{\vee} \rightarrow A\right)$ and $S_{0}:=\operatorname{Spec}\left(A_{0}\right)$. In other words, $S_{0}$ is the zero set of the section $\mu$. If $S_{0}$ is empty, then $f$ is an isomorphism and the lemma becomes trivial. So let us assume that $S_{0}$ is nonempty. Let $\mathcal{R}$ be defined by the cartesian diagram

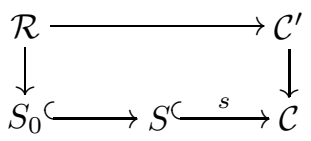

Then $\mathcal{R}=\operatorname{Proj}(\operatorname{Sym}(H))$ for a free $A_{0}$-module $H$ of rank two. Let $\pi_{0}^{\prime}: \mathcal{R} \rightarrow S_{0}$ be the projection, $s_{0}^{\prime}: S_{0} \rightarrow \mathcal{R}$ the section of $\pi_{0}^{\prime}$ induced by $s^{\prime}$, and let $\mathcal{E}_{\mathcal{R}}^{\prime}$ be the restriction of $\mathcal{E}^{\prime}$ to $\mathcal{R}$. The admissibility of $\mathcal{E}^{\prime}$ implies the vanishing of $H^{1}\left(\mathcal{R}_{x},\left.\mathcal{E}_{\mathcal{R}}^{\prime}\left(-s^{\prime}\right)\right|_{\mathcal{R}_{x}}\right)$ for every fiber $\mathcal{R}_{x}$ of $\pi_{0}^{\prime}$. From this and the flatness of $\pi_{0}^{\prime}$ it follows that the sheaf $\mathcal{E}_{\mathcal{R}, 1}^{\prime}:=\left(\pi_{0}^{\prime}\right)^{*}\left(\left(\pi_{0}^{\prime}\right)_{*} \mathcal{E}_{\mathcal{R}}^{\prime}\left(-s_{0}^{\prime}\right)\right)\left(s_{0}^{\prime}\right)$ is locally free and its formation commutes with base change. The adjunction morphism gives us a canonical morphism $\mathcal{E}_{\mathcal{R}, 1}^{\prime} \rightarrow \mathcal{E}_{\mathcal{R}}^{\prime}$. Let $\mathcal{E}_{\mathcal{R}, 2}^{\prime}$ be the cokernel of this morphism. By restricting to fibers one finds that the sequence

$$
0 \rightarrow \mathcal{E}_{\mathcal{R}, 1}^{\prime} \rightarrow \mathcal{E}_{\mathcal{R}}^{\prime} \rightarrow \mathcal{E}_{\mathcal{R}, 2}^{\prime} \rightarrow 0
$$

is exact and that

$$
\left.\left(\mathcal{E}_{\mathcal{R}, 1}^{\prime}\right)\right|_{\mathcal{R}_{x}} \cong \bigoplus^{d} \mathcal{O}_{\mathcal{R}_{x}}(1) \quad,\left.\quad\left(\mathcal{E}_{\mathcal{R}, 2}^{\prime}\right)\right|_{\mathcal{R}_{x}} \cong \bigoplus^{n-d} \mathcal{O}_{\mathcal{R}_{x}}
$$

for all $x \in S_{0}$. Now we choose a direct sum decomposition $\left(s^{\prime}\right)^{*} \mathcal{E}^{\prime} \stackrel{\sim}{\rightarrow}\left(M^{\vee} \otimes E_{1}\right) \oplus E_{2}$ compatible with the above exact sequence, i.e. in such a way that there exist isomorphisms $\left.\left(s_{0}^{\prime}\right)^{*} \mathcal{E}_{\mathcal{R}, 1} \stackrel{\sim}{\rightarrow}\left(M^{\vee} \otimes E_{1}\right)\right|_{S_{0}}$ and $\left.\left(s_{0}^{\prime}\right)^{*} \mathcal{E}_{\mathcal{R}, 2} \stackrel{\sim}{\rightarrow} E_{2}\right|_{S_{0}}$ which make the following diagram commute:

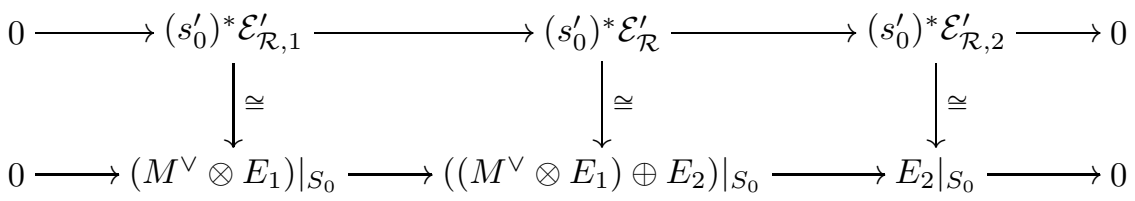

where the middle vertical arrow is the one induced from $\left(s^{\prime}\right)^{*} \mathcal{E}^{\prime} \stackrel{\sim}{\rightarrow}\left(M^{\vee} \otimes E_{1}\right) \oplus E_{2}$ by restriction to $S_{0}$. Define the locally free $\mathcal{O}_{\mathcal{C}^{\prime}}$-module $\mathcal{F}^{\prime}$ by the exact sequence

$$
0 \rightarrow \mathcal{F}^{\prime} \rightarrow \mathcal{E}^{\prime} \rightarrow s_{*}^{\prime}\left(M^{\vee} \otimes E_{1}\right) \rightarrow 0
$$

and let $\mathcal{F}:=f_{*} \mathcal{F}^{\prime}$. It is easy to see that $\mathcal{F}^{\prime}$ is trivial along the fibers of $f$. By $[\mathrm{Kn}$, Cor. 1.5, the sheaf $\mathcal{F}$ is flat over $S$ and its formation commutes with base change. It is easy to see that the restriction of $\mathcal{F}$ to fibers of $\pi$ is locally free of rank $n$. In particular, all the fibers $\mathcal{F}[x]$ are of dimension $n$.

I claim that $\mathcal{F}$ itself is locally free of rank $n$. To prove this, let $I \in A$ be the nilradical of $A$ and let $J:=I B$. Then $\bar{S}:=S_{\text {red }}=\operatorname{Spec}(A / I)$ and $\overline{\mathcal{C}}:=\mathcal{C} \times{ }_{S} S_{\text {red }}=$ $\operatorname{Spec}(B / J)$. By $[\mathrm{Ma}], 22.3(3)$, it suffices to show that $J \otimes_{B} \mathcal{F}=J \cdot \mathcal{F}$ and that $\left.\mathcal{F}\right|_{\overline{\mathcal{C}}}$ is a flat $\mathcal{O}_{\overline{\mathcal{C}}}$-module. The first of these conditions follows easily from the flatness of $B / A$ and $\mathcal{F} / A$. Since $\overline{\mathcal{C}} \rightarrow \bar{S}$ is smooth and $\bar{S}$ is reduced, $\overline{\mathcal{C}}$ is also reduced (cf. SGA1, Exp. II, Prop 3.1). As observed above, all fibers of $\mathcal{F}$ (and thus of $\left.\mathcal{F}\right|_{\overline{\mathcal{C}}}$ ) 
have the same dimension $n$. But a coherent sheaf over a reduced locally noetherian scheme all of whose fibres have the same dimension is locally free (cf. [Mi], 2.9, where the ground ring is assumed to be integer, but the proof works as well for reduced ground ring). This proves our claim.

I claim furthermore that the canonical morphism $f^{*} \mathcal{F} \rightarrow \mathcal{F}^{\prime}$ is an isomorphism. Indeed, since $\mathcal{F}^{\prime}$ is flat over $S$, it suffices to check this on the fibers of $\pi^{\prime}$, which is easy.

By applying $\left(s^{\prime}\right)^{*}$ to the defining sequence for $\mathcal{F}^{\prime}$ we obtain the exact sequence

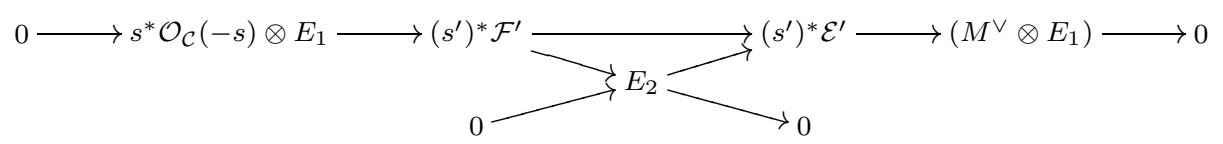

Since we have $s^{*} \mathcal{F}=\left(s^{\prime}\right)^{*} f^{*} \mathcal{F}=\left(s^{\prime}\right)^{*} \mathcal{F}^{\prime}$, we get the exact sequence

$$
0 \rightarrow s^{*} \mathcal{O}_{\mathcal{C}}(-s) \otimes E_{1} \rightarrow s^{*} \mathcal{F} \rightarrow E_{2} \rightarrow 0
$$

After replacing $S$ and $\mathcal{C}$ by members of an open covering, we can assume that there exists a decomposition $\mathcal{F} \stackrel{\sim}{\rightarrow} \mathcal{E}_{1}(-s) \oplus \mathcal{E}_{2}$ together with isomorphisms $E_{1} \stackrel{\sim}{\rightarrow} s^{*} \mathcal{E}_{1}$ and $E_{2} \stackrel{\sim}{\rightarrow} s^{*} \mathcal{E}_{2}$ which is compatible with this exact sequence. Observe that we have

$$
\begin{aligned}
\operatorname{ker}\left(\mathcal{F}^{\prime} \rightarrow s_{*}^{\prime} E_{2}\right) \otimes_{\mathcal{O}_{\mathcal{C}^{\prime}}} \mathcal{O}_{\mathcal{C}^{\prime}}\left(s^{\prime}\right) & \cong \operatorname{ker}\left(f^{*} \mathcal{F} \rightarrow s_{*}^{\prime} E_{2}\right) \otimes_{\mathcal{O}_{\mathcal{C}^{\prime}}} \mathcal{O}_{\mathcal{C}^{\prime}}\left(s^{\prime}\right) \\
& \cong\left(\mathcal{M}^{\vee} \otimes f^{*} \mathcal{E}_{1}\right) \oplus f^{*} \mathcal{E}_{2}=\mathcal{E}^{\prime}\left(\mathcal{E}_{1}, \mathcal{E}_{2}\right)
\end{aligned}
$$

On the other hand, the exact diagram

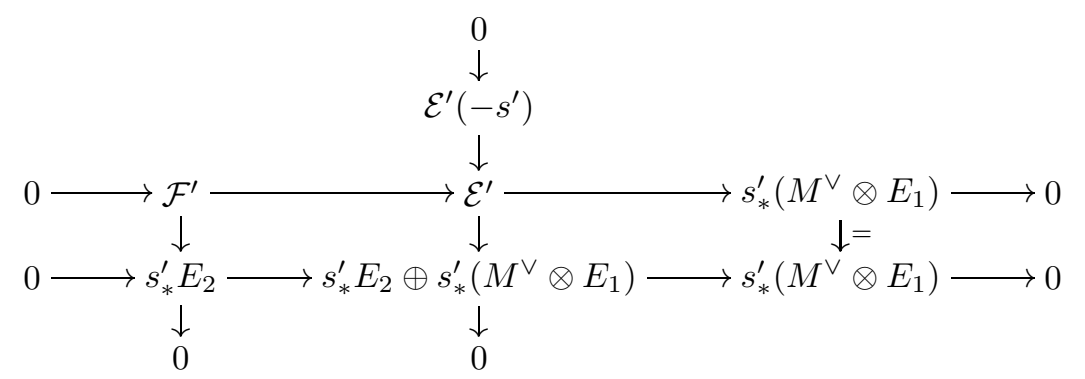

shows that $\operatorname{ker}\left(\mathcal{F}^{\prime} \rightarrow s_{*}^{\prime} E_{2}\right) \otimes_{\mathcal{O}_{\mathcal{C}^{\prime}}} \mathcal{O}_{\mathcal{C}^{\prime}}\left(s^{\prime}\right) \cong \mathcal{E}^{\prime}$. Therefore $\mathcal{E}^{\prime}\left(\mathcal{E}_{1}, \mathcal{E}_{2}\right) \cong \mathcal{E}^{\prime}$.

From Lemmas 7.5 and 7.6 it follows easily that the tuple $\left(M, \mu, E^{\prime} \rightarrow E, M \otimes\right.$

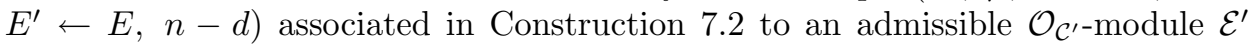
of degree $d$ and isomorphisms $\left(f_{*} \mathcal{E}^{\prime}\left(-s^{\prime}\right)\right)(s) \stackrel{\sim}{\rightarrow} \mathcal{E}$ and $\left(s^{\prime}\right)^{*} \mathcal{E}^{\prime} \stackrel{\sim}{\rightarrow} E^{\prime}$ is indeed a bf-morphism of rank $n-d$ from $E^{\prime}$ to $E$ in the sense of [K1], 3.1. Thus the arrow in Proposition 7.4 is well defined.

Our aim is now to define the inverse of Construction 7.2 Let

$$
\left(M, \mu, E^{\prime} \rightarrow E, M \otimes E^{\prime} \leftarrow E, n-d\right)
$$

be an arbitrary bf-morphism of degree $n-d$ from $E^{\prime}$ to $E$.

Assume first that $S$ and $\mathcal{C}$ are affine, that $M$ is trivial and that there exist free $\mathcal{O}_{\mathcal{C}}$-modules $\mathcal{E}_{1}$ and $\mathcal{E}_{2}$ of rank $d$ and $n-d$ respectively, and isomorphisms

$$
\begin{array}{rll}
\varphi & : & \mathcal{E}_{1} \oplus \mathcal{E}_{2} \stackrel{\sim}{\longrightarrow} \mathcal{E} \\
\varphi^{\prime} & : & \left(M^{\vee} \otimes E_{1}\right) \oplus E_{2} \stackrel{\sim}{\longrightarrow} E^{\prime}
\end{array}
$$


where $E_{1}:=s^{*} \mathcal{E}_{1}$ and $E_{2}:=s^{*} \mathcal{E}_{2}$, such that the following diagram commutes:

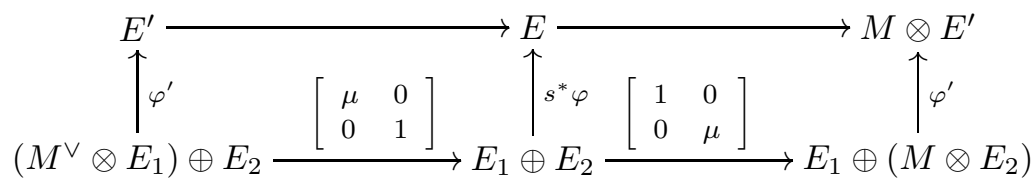

Let $\mathcal{E}^{\prime}:=\mathcal{E}^{\prime}\left(\mathcal{E}_{1}, \mathcal{E}_{2}\right)$. Composing $\varphi$ and $\varphi^{\prime}$ with the natural isomorphisms of Lemma 7.5 we get isomorphisms which we denote again by the same symbols:

$$
\begin{aligned}
\varphi & :\left(f_{*} \mathcal{E}^{\prime}\left(-s^{\prime}\right)\right)(s) \stackrel{\sim}{\longrightarrow} \mathcal{E} \\
\varphi^{\prime} & : \quad\left(s^{\prime}\right)^{*} \mathcal{E}^{\prime} \stackrel{\sim}{\longrightarrow} E^{\prime}
\end{aligned}
$$

If

$$
\begin{aligned}
\psi: & \mathcal{E}_{1} \oplus \mathcal{E}_{2} \stackrel{\sim}{\longrightarrow} \mathcal{E} \\
\psi^{\prime} & : \quad\left(M^{\vee} \otimes E_{1}\right) \oplus E_{2} \stackrel{\sim}{\longrightarrow} E^{\prime}
\end{aligned}
$$

is another pair of isomorphisms such that the diagram analogous to $(*)$ commutes, then we get in the same way a pair of isomorphisms

$$
\begin{aligned}
\psi & : \quad\left(f_{*} \mathcal{E}^{\prime}\left(-s^{\prime}\right)\right)(s) \stackrel{\sim}{\longrightarrow} \mathcal{E} \\
\psi^{\prime} & :\left(s^{\prime}\right)^{*} \mathcal{E}^{\prime} \stackrel{\sim}{\longrightarrow} E^{\prime}
\end{aligned}
$$

Lemma 7.7. In the above situation there exists a unique isomorphism $g: \mathcal{E}^{\prime} \stackrel{\sim}{\longrightarrow} \mathcal{E}^{\prime}$ such that the following diagrams commute:

$$
\left(f_{*} \mathcal{E}^{\prime}\left(-s^{\prime}\right)\right)(s) \stackrel{\cong}{\longrightarrow}\left(f_{*} \mathcal{E}^{\prime}\left(-s^{\prime}\right)\right)(s) \quad\left(s^{\prime}\right)^{*} \mathcal{E}^{\prime} \stackrel{\varphi^{\prime}{ }_{\mathcal{E}^{\prime}} \cong}{\longrightarrow}\left(s^{\prime}\right)^{*} \mathcal{E}^{\prime}
$$

where the horizontal arrows are the ones induced by $g$.

Before proving Lemma 7.7, we will establish the following auxiliary result:

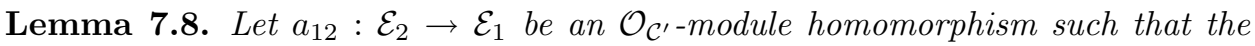
induced morphism $\alpha_{12}: E_{2} \rightarrow E_{1}$ factorizes as follows:

$$
E_{2} \stackrel{\beta_{12}}{\longrightarrow} M^{\vee} \otimes E_{1} \stackrel{\mu}{\longrightarrow} E_{1}
$$

Then there exists a factorization

$$
f^{*} \mathcal{E}_{2} \stackrel{b_{12}}{\longrightarrow} \mathcal{M}^{\vee} \otimes f^{*} \mathcal{E}_{1} \longrightarrow f^{*} \mathcal{E}_{1}
$$

of $f^{*} a_{12}$, such that $\left(s^{\prime}\right)^{*} b_{12}=\beta_{12}$. Here the morphism $\mathcal{M}^{\vee} \otimes f^{*} \mathcal{E}_{1} \rightarrow f^{*} \mathcal{E}_{1}$ is the one induced by the arrow $m: \mathcal{O}_{\mathcal{C}^{\prime}} \rightarrow \mathcal{M}$ of Construction 5.2 .

Proof. After choosing a bases of $\mathcal{E}_{1}$ and of $\mathcal{E}_{2}$ one is easily reduced to the case $\mathcal{E}_{1}=\mathcal{E}_{2}=\mathcal{O}_{\mathcal{C}^{\prime}}$. Let $\mathcal{I}$ be the $\mathcal{O}_{\mathcal{C}}$-module associated to the pair $(M, \mu)$ as in Construction 5.4, Consider the morphism

$$
\pi^{*} M^{\vee} \oplus \mathcal{O}_{\mathcal{C}}(-s) \rightarrow \mathcal{O}_{\mathcal{C}}
$$

which is the sum of the morphism $\pi^{*} M^{\vee} \rightarrow \mathcal{O}_{\mathcal{C}}$ induced by $\mu$ and the inclusion $\mathcal{O}_{\mathcal{C}}(-s) \hookrightarrow \mathcal{O}_{\mathcal{C}}$. Obviously, this morphism factorizes through $\mathcal{I}$. Thus we have a natural morphism $m: \mathcal{I} \rightarrow \mathcal{O}_{\mathcal{C}}$ and this induces a morphism $\mathcal{S}(1) \rightarrow \mathcal{S}$ of graded $\mathcal{S}$-modules, where $\mathcal{S}$ is the graded $\mathcal{O}_{\mathcal{C}}$-algebra $\mathcal{S}=\operatorname{Sym}(\mathcal{I})$. Let $\mathcal{M}:=\mathcal{O}_{\mathcal{C}^{\prime}}\left(-s^{\prime}\right) \otimes$ 
$f^{*} \mathcal{O}_{\mathcal{C}}(s)$. We have seen on page 4924 that $\mathcal{O}_{\mathcal{C}^{\prime}}(1)=\mathcal{M}^{\vee}$. It is not difficult to see that under this identification the morphism $\mathcal{O}_{\mathcal{C}^{\prime}}(1) \rightarrow \mathcal{O}_{\mathcal{C}^{\prime}}$ induced by $\mathcal{S}(1) \rightarrow \mathcal{S}$ corresponds to the morphism dual to $\mathcal{O}_{\mathcal{C}^{\prime}} \rightarrow \mathcal{M}$ defined in Construction [5.2. Let $S=\operatorname{Spec}(A), \mathcal{C}=\operatorname{Spec}(B)$ for a $\operatorname{ring} A$ and an $A$-algebra $B$ (remember that we are still dealing with the affine case). We are given a $B$-module morphism $a_{12}: B \rightarrow B$ such that the induced morphism $\alpha_{12}: A \rightarrow A$ factorizes as $A \stackrel{\beta_{12}}{\rightarrow} M^{\vee} \stackrel{\mu}{\rightarrow} A$. We have to establish a factorization $B \rightarrow \mathcal{I} \stackrel{m}{\rightarrow} B$ such that the following diagram commutes:

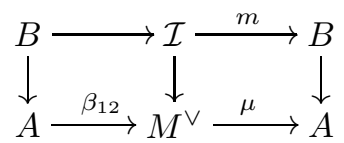

where the middle vertical arrow is the one defining $s^{\prime}$ (cf. Construction 5.4). Let $b \in B$ be a generator of $\operatorname{ker}(B \rightarrow A)$ and let $l: M \stackrel{\sim}{\rightarrow} A$ be an isomorphism. By assumption we have $a_{12}(1)=b_{1} l(\mu)+b_{2} b$ for some $b_{1}, b_{2} \in B$. Furthermore we may choose $b_{1}, b_{2}$ in such a way that $\overline{b_{1}} l=\beta_{12}(1)$, where $\overline{b_{1}} \in A$ is the image of $b_{1}$ under the surjection $B \rightarrow A$. By definition, we may identify $\mathcal{I}$ with the $B$-module $(B \cdot X \oplus B \cdot Y) /(b X-l(\mu) Y)$. With this identification the morphism $\mathcal{I} \rightarrow M^{\vee}$ is given by $X \mapsto l, Y \mapsto 0$ and the morphism $m: \mathcal{I} \rightarrow B$ is given by $X \mapsto l(\mu)$, $Y \mapsto b$. Therefore the morphism $B \rightarrow \mathcal{I}$ defined by $1 \mapsto b_{1} X+b_{2} Y$ yields the required factorization.

Proof of Lemma 7.7. Let

$$
\begin{aligned}
& {\left[\begin{array}{ll}
a_{11} & a_{12} \\
a_{21} & a_{22}
\end{array}\right]:=\psi \circ \varphi^{-1}: \mathcal{E}_{1} \oplus \mathcal{E}_{2} \stackrel{\sim}{\rightarrow} \mathcal{E}_{1} \oplus \mathcal{E}_{2}} \\
& {\left[\begin{array}{ll}
\alpha_{11} & \alpha_{12} \\
\alpha_{21} & \alpha_{22}
\end{array}\right]:=s^{*}\left(\psi \circ \varphi^{-1}\right): E_{1} \oplus E_{2} \stackrel{\sim}{\rightarrow} E_{1} \oplus E_{2}} \\
& {\left[\begin{array}{ll}
\beta_{11} & \beta_{12} \\
\beta_{21} & \beta_{22}
\end{array}\right]:=\psi^{\prime} \circ\left(\varphi^{\prime}\right)^{-1}:\left(M^{\vee} \otimes E_{1}\right) \oplus E_{2} \stackrel{\sim}{\rightarrow}\left(M^{\vee} \otimes E_{1}\right) \oplus E_{2}}
\end{aligned}
$$

Let $\mathcal{E}_{1}^{\prime}:=\mathcal{M}^{\vee} \otimes f^{*} \mathcal{E}_{1}$ and $\mathcal{E}_{2}^{\prime}:=f^{*} \mathcal{E}_{2}$. Then $\mathcal{E}^{\prime}=\mathcal{E}_{1}^{\prime} \oplus \mathcal{E}_{2}^{\prime}$ and a morphism $\mathcal{E}^{\prime} \rightarrow \mathcal{E}^{\prime}$ is of the form

$$
\left[\begin{array}{ll}
b_{11} & b_{12} \\
b_{21} & b_{22}
\end{array}\right]: \mathcal{E}_{1}^{\prime} \oplus \mathcal{E}_{2}^{\prime} \rightarrow \mathcal{E}_{1}^{\prime} \oplus \mathcal{E}_{2}^{\prime}
$$

We have to show that there exist morphisms $b_{i j} \in \operatorname{Hom}\left(\mathcal{E}_{j}^{\prime}, \mathcal{E}_{i}^{\prime}\right)$ with

$$
f_{*}\left(\mathcal{M} \otimes b_{i j}\right)=a_{i j} \quad \text { and } \quad\left(s^{\prime}\right)^{*} b_{i j}=\beta_{i j}
$$

for $i, j \in\{1,2\}$ and that they are uniquely determined by this property.

We let $b_{11}:=\mathcal{M}^{\vee} \otimes f^{*} a_{11}$ and $b_{22}:=f^{*} a_{22}$ and define $b_{21}$ as the composition

$$
b_{21}: \mathcal{M}^{\vee} \otimes f^{*} \mathcal{E}_{1} \stackrel{m}{\longrightarrow} f^{*} \mathcal{E}_{1} \stackrel{f^{*} a_{21}}{\longrightarrow} f^{*} \mathcal{E}_{2}
$$

It is easy to check that the commutativity of the diagram $(*)$ on page 4936 implies

$$
\begin{array}{cc}
\alpha_{11}=\beta_{11} & \alpha_{12}=\mu \beta_{12} \\
\mu \alpha_{21}=\beta_{21} & \alpha_{22}=\beta_{22}
\end{array}
$$

By Lemma 7.8 there exists a morphism $b_{12}: f^{*} \mathcal{E}_{2} \rightarrow \mathcal{M}^{\vee} \otimes f^{*} \mathcal{E}_{1}$ with $m b_{12}=f^{*} a_{12}$ and $\left(s^{\prime}\right)^{*} b_{12}=\beta_{12}$. One checks without difficulty that the $b_{i j}$ thus defined have the 
required property. We will see below that the matrix $\left[b_{i j}\right]$ defines an isomorphism $\mathcal{E}^{\prime} \stackrel{\sim}{\rightarrow} \mathcal{E}^{\prime}$.

The uniqueness of $b_{i j}$ is clear for $(i, j) \in\{(1,1),(2,1),(2,2)\}$, since in these cases the morphism $\operatorname{Hom}\left(\mathcal{E}_{j}^{\prime}, \mathcal{E}_{i}^{\prime}\right) \rightarrow \operatorname{Hom}\left(\mathcal{E}_{j}, \mathcal{E}_{i}\right)$ given by $g \mapsto f_{*}(\mathcal{M} \otimes g)$ is an isomorphism. For the uniqueness of $b_{12}$ we have to show the injectivity of the morphism

$$
v^{\prime}:\left\{\begin{array}{cll}
\operatorname{Hom}\left(f^{*} \mathcal{E}_{2}, \mathcal{M}^{\vee} \otimes f^{*} \mathcal{E}_{1}\right) & \rightarrow & \operatorname{Hom}\left(\mathcal{E}_{2}, \mathcal{E}_{1}\right) \\
g & \mapsto \quad \operatorname{Hom}\left(E_{2}, M^{\vee} \otimes E_{1}\right) \\
& \left.\mapsto\left(f_{*} \otimes g\right),\left(s^{\prime}\right)^{*} g\right)
\end{array}\right.
$$

Let $v_{1}: f_{*} \mathcal{M}^{\vee} \rightarrow \mathcal{O}_{\mathcal{C}}$ and $v_{2}: f_{*} \mathcal{M}^{\vee} \rightarrow s_{*} M^{\vee}$ be the indicated arrows in the commutative diagram with exact rows

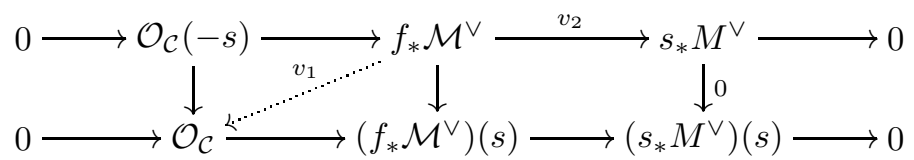

We have canonical identifications

$$
\begin{aligned}
& \operatorname{Hom}\left(f^{*} \mathcal{E}_{2}, \mathcal{M}^{\vee} \otimes f^{*} \mathcal{E}_{1}\right)=\operatorname{Hom}\left(\mathcal{E}_{2}, \mathcal{E}_{1}\right) \otimes f_{*} \mathcal{M}^{\vee} \\
\text { and } & \operatorname{Hom}\left(E_{2}, M^{\vee} \otimes E_{1}\right)=\operatorname{Hom}\left(\mathcal{E}_{2}, \mathcal{E}_{1}\right) \otimes_{\mathcal{O}_{\mathcal{C}}} s_{*} M^{\vee}
\end{aligned}
$$

and it is not difficult to see that the morphism $v^{\prime}$ coincides with the morphism

$$
\operatorname{Hom}\left(\mathcal{E}_{2}, \mathcal{E}_{1}\right) \otimes f_{*} \mathcal{M}^{\vee} \rightarrow \operatorname{Hom}\left(\mathcal{E}_{2}, \mathcal{E}_{1}\right) \otimes\left(\mathcal{O}_{\mathcal{C}} \oplus s_{*} M^{\vee}\right)
$$

obtained by tensoring $v:=v_{1}+v_{2}: f_{*} \mathcal{M}^{\vee} \rightarrow \mathcal{O}_{\mathcal{C}} \oplus s_{*} M^{\vee}$ with $\operatorname{Hom}\left(\mathcal{E}_{2}, \mathcal{E}_{1}\right)$. Therefore the injectivity of $v^{\prime}$ is equivalent to the injectivity of $v$. But the injectivity of $v$ is clear, since

$$
\operatorname{ker}(v)=\operatorname{ker}\left(v_{1}\right) \cap \operatorname{ker}\left(v_{2}\right)=\operatorname{ker}\left(\mathcal{O}_{\mathcal{C}}(-s) \rightarrow \mathcal{O}_{\mathcal{C}}\right)=0 .
$$

It is now easy to see that the morphism $g: \mathcal{E}^{\prime} \rightarrow \mathcal{E}^{\prime}$ defined by the matrix $\left[b_{i j}\right]$ is an isomorphism: Let $g^{\prime}: \mathcal{E}^{\prime} \rightarrow \mathcal{E}^{\prime}$ be the morphism constructed in the same way as $g$, only with the roles of $\left(\varphi, \varphi^{\prime}\right)$ and $\left(\psi, \psi^{\prime}\right)$ interchanged. By uniqueness it follows that $g \circ g^{\prime}=g^{\prime} \circ g=$ id.

Construction 7.9. We return now to the general case and let

$$
\left(M, \mu, E^{\prime} \rightarrow E, M \otimes E^{\prime} \leftarrow E, n-d\right)
$$

be a bf-morphism of rank $n-d$ from $E^{\prime}$ to $E$. With the help of Lemma 4.1 in K1 it is easy to see that there exists an open affine covering $\mathcal{C}=U_{0} \cup \bigcup_{i \in I} U_{i}$ with the following properties:

(1) $U_{0}$ is the complement of the section $s$ in $\mathcal{C}$.

(2) For each $i \in I, s$ induces a section of $\left.\pi\right|_{U_{i}}: U_{i} \rightarrow \pi\left(U_{i}\right)$. In particular, $\pi\left(U_{i}\right)$ is affine.

(3) For each $i \in I$ the invertible sheaf $M$ is trivial over $\pi\left(U_{i}\right)$.

(4) For each $i \in I$ there exist free $\mathcal{O}_{U_{i}}$-modules $\mathcal{E}_{1}^{i}$ and $\mathcal{E}_{2}^{i}$ of rank $d$ and $n-d$ respectively, and isomorphisms

$$
\begin{array}{ll}
\varphi_{i}: & \left.\mathcal{E}_{1}^{i} \oplus \mathcal{E}_{2}^{i} \stackrel{\sim}{\longrightarrow} \mathcal{E}\right|_{U_{i}} \\
\varphi_{i}^{\prime} & :\left.\quad\left(M^{\vee} \otimes E_{1}^{i}\right) \oplus E_{2}^{i} \stackrel{\sim}{\longrightarrow} E^{\prime}\right|_{s^{-1}\left(U_{i}\right)}
\end{array}
$$

where $E_{1}^{i}:=s^{*} \mathcal{E}_{1}^{i}$ and $E_{2}^{i}:=s^{*} \mathcal{E}_{2}^{i}$, such that over $s^{-1}\left(U_{i}\right)$ the diagram analogous to $(*)$ (cf. page 4936) commutes. 
For $i \in I$ we have over $V_{i}:=f^{-1}\left(U_{i}\right)$ the locally free $\mathcal{O}_{V_{i}}$-modules

$$
\mathcal{E}_{i}^{\prime}:=\mathcal{E}^{\prime}\left(\mathcal{E}_{1}^{i}, \mathcal{E}_{2}^{i}\right)=\left(\mathcal{O}_{\mathcal{C}^{\prime}}\left(s^{\prime}\right) \otimes f^{*} \mathcal{E}_{1}^{i}(-s)\right) \oplus f^{*} \mathcal{E}_{2}^{i}
$$

together with the isomorphisms

$$
\begin{aligned}
& \varphi_{i}:\left(f_{*} \mathcal{E}_{i}^{\prime}\left(-s^{\prime}\right)\right)(s) \stackrel{\sim}{\longrightarrow} \mathcal{E} \mid U_{i} \\
& \varphi_{i}^{\prime}:\left.\left(s^{\prime}\right)^{*} \mathcal{E}_{i}^{\prime} \stackrel{\sim}{\longrightarrow} E^{\prime}\right|_{s^{-1}\left(U_{i}\right)}
\end{aligned}
$$

Let $U:=\bigcup_{i \in I} U_{i}$ and $V:=f^{-1}(U)$. Then $\left(V,\left.f\right|_{V},\left.\pi\right|_{U}, s\right)$ is a simple modification of $\left(U,\left.\pi\right|_{U}, s\right)$ and by Lemmas 7.5 and 7.7 the data $\left(\mathcal{E}_{i}^{\prime}, \varphi_{i}, \varphi_{i}^{\prime}\right)$ glue together to give an admissible $\mathcal{O}_{V}$-module $\mathcal{E}_{V}^{\prime}$ of degree $d$ together with isomorphisms $\varphi_{V}$ : $\left.\left(\left(\left.f\right|_{V}\right)_{*} \mathcal{E}_{V}^{\prime}\left(-s^{\prime}\right)\right)(s) \stackrel{\sim}{\longrightarrow} \mathcal{E}\right|_{U}$ and $\varphi^{\prime}:\left(s^{\prime}\right)^{*} \mathcal{E}_{V}^{\prime} \stackrel{\sim}{\longrightarrow} E^{\prime}$. Let $V_{0}:=f^{-1}\left(U_{0}\right)$ and $\mathcal{E}_{0}^{\prime}:=\left(\left.f\right|_{V_{0}}\right)^{*} \mathcal{E}$. Since the restriction of $f$ to $V_{0}$ is an isomorphism onto $U_{0}$, we may identify $V_{0}$ with $U_{0}, \mathcal{E}_{0}^{\prime}$ with $\left.\mathcal{E}\right|_{U_{0}}$, and $\left.\left(\left(\left.f\right|_{V}\right)_{*} \mathcal{E}_{V}^{\prime}\left(-s^{\prime}\right)\right)(s)\right|_{V_{0} \cap V}$ with $\left.\mathcal{E}_{V}^{\prime}\right|_{V_{0} \cap V}$. Thus $\mathcal{E}_{V}^{\prime}$ and $\mathcal{E}_{0}^{\prime}$ glue together via $\varphi_{V}$ to give an admissible $\mathcal{O}_{\mathcal{C}^{\prime}}$-module $\mathcal{E}^{\prime}$ of degree $d$ together with isomorphisms $\left(f_{*} \mathcal{E}^{\prime}\left(-s^{\prime}\right)\right)(s) \stackrel{\sim}{\longrightarrow} \mathcal{E}$ and $\left(s^{\prime}\right)^{*} \mathcal{E}^{\prime} \stackrel{\sim}{\longrightarrow} E^{\prime}$. By Lemma 7.7, this construction does not depend on the choice of the $U_{i}, \varphi_{i}, \varphi_{i}^{\prime}$.

Remark 7.10. Construction 7.9 has the drawback that it involves a choice of the data $U_{i}, \varphi_{i}, \varphi_{i}^{\prime}$. I have tried in vain to find a direct construction, which would produce a canonical graded $\operatorname{Sym}(\mathcal{I})$-module out of a bf-morphism from $E^{\prime}$ to $E$ such that the associated $\mathcal{O}_{\mathcal{C}^{\prime}}$-module would be $\mathcal{E}^{\prime}$.

It is an immediate consequence of Lemmas 7.5, 7.6 and 7.7 that Constructions 7.2 and 7.9 are inverses of each other. This proves Proposition 7.4.

\section{Contractions}

The following proposition is implicit in the proof of Theorem $2.1 \mathrm{in}$ [n].

Proposition 8.1. Let $Y$ be a locally noetherian scheme, let $\pi: X \rightarrow Y$ be a proper flat morphism with $\pi_{*} \mathcal{O}_{X}=\mathcal{O}_{Y}$ and let $\mathcal{L}$ be an invertible $\mathcal{O}_{X}$-module. For $y \in Y$ we denote by $X_{y}$ the fibre of $\pi$ over $y$ and by $\mathcal{L}_{y}$ the restriction of $\mathcal{L}$ to $X_{y}$. Assume that for every $y \in Y$ the following holds:

(1) $H^{1}\left(X_{y}, \mathcal{L}_{y}^{\otimes i}\right)=(0)$ for $i \geq 1$.

(2) The $\mathcal{O}_{X_{y}}$-module $\mathcal{L}_{y}$ is generated by global sections.

(3) For every $i \geq 1$ the canonical morphism $\left(H^{0}\left(X_{y}, \mathcal{L}_{y}\right)\right)^{\otimes i} \rightarrow H^{0}\left(X_{y}, \mathcal{L}_{y}^{\otimes i}\right)$ is surjective.

Let $\mathcal{S}$ be the graded $\mathcal{O}_{Y}$-algebra $\bigoplus_{i \geq 0} \pi_{*} \mathcal{L}^{\otimes i}$ and let $X^{c}:=\operatorname{Proj}(\mathcal{S})$. Then the canonical morphism $\pi^{*} \pi_{*} \mathcal{L} \rightarrow \mathcal{L}$ (resp. Sym $\pi_{*} \mathcal{L} \rightarrow \mathcal{S}$ ) is surjective and therefore induces a morphism $p: X \rightarrow \operatorname{Proj}\left(\operatorname{Sym} \pi_{*} \mathcal{L}\right)$ (resp. a closed immersion $X^{c} \hookrightarrow$ $\left.\operatorname{Proj}\left(\operatorname{Sym} \pi_{*} \mathcal{L}\right)\right)$. The scheme theoretic image of $p$ is $X^{c}$ and $X^{c}$ is flat over $Y$.

Proof. We set $\mathcal{S}^{i}:=\pi_{*} \mathcal{L}^{\otimes i}$ for $i \geq 0$. It is well known that property (1) ensures that the sheaves $\mathcal{S}^{i}$ are locally free and that their construction commutes with any base change $Y^{\prime} \rightarrow Y$. By definition, $\mathcal{S}$ is the graded algebra with components $\mathcal{S}^{i}$. An appplication of Nakayama's lemma shows that property (2) and property (3) entails the surjectivity of $\pi^{*} \mathcal{S}^{1} \rightarrow \mathcal{L}$ and of $\operatorname{Sym} \mathcal{S}^{1} \rightarrow \mathcal{S}$, respectively. The first of these surjections induces a morphism $p: X \rightarrow \operatorname{Proj}\left(\operatorname{Sym} \mathcal{S}^{1}\right.$ ) (cf. [EGA II], (4.3.2)), and the second induces a closed immersion $X^{c} \hookrightarrow \operatorname{Proj}\left(\operatorname{Sym} \mathcal{S}^{1}\right)$ (cf. [EGA II], $(2.9 .2)(\mathrm{i}))$. 
To prove that $X^{c}$ is the scheme-theoretic image of $p$, we may assume that $Y$ is affine. Let $S^{i}:=\Gamma\left(Y, \mathcal{S}^{i}\right)$ and $S:=\Gamma(Y, \mathcal{S})$. For $f \in S^{1}$ we consider the open subscheme $U_{f}:=\operatorname{Spec}\left(\operatorname{Sym} S^{1}\right)_{(f)}$ of $\operatorname{Proj}\left(\operatorname{Sym} S^{1}\right)$. Its preimage under $p: X \rightarrow \operatorname{Proj}\left(\operatorname{Sym} S^{1}\right)$ is $X_{f}:=\{x \in X \mid f(x) \neq 0\}$ and its preimage under $X^{c} \hookrightarrow \operatorname{Proj}\left(\operatorname{Sym} S^{1}\right)$ is $V_{f}:=\operatorname{Spec}\left(S_{(f)}\right)$. The restriction of $f$ to $X_{f}$ defines a trivialization $\left.\mathcal{L}\right|_{X_{f}} \stackrel{\sim}{\rightarrow} \mathcal{O}_{X_{f}}$ and thus a morphism $S^{1}=\Gamma(X, \mathcal{L}) \rightarrow \Gamma\left(X_{f}, \mathcal{L}\right) \stackrel{\sim}{\rightarrow}$ $\Gamma\left(X_{f}, \mathcal{O}_{X}\right)$ which maps $f$ to 1 . The induced morphism $\left(\operatorname{Sym} S^{1}\right)_{(f)} \rightarrow \Gamma\left(X_{f}, \mathcal{O}_{X}\right)$ is the defining one for the morphism $\left.p\right|_{X_{f}}: X_{f} \rightarrow U_{f} \subset \operatorname{Proj}\left(\operatorname{Sym} S^{1}\right)$. We have a canonical morphism

$$
\begin{cases}S_{(f)} & \longrightarrow \Gamma\left(X_{f}, \mathcal{O}_{X}\right) \\ a / f^{n} & \longrightarrow b\end{cases}
$$

where $a \in S^{n}=\Gamma\left(X, \mathcal{L}^{\otimes n}\right)$ and $b \in \Gamma\left(X_{f}, \mathcal{O}_{X}\right)$ is the element defined by the relation $\left.a\right|_{X_{f}}=\left.b \cdot f^{n}\right|_{X_{f}} \in \Gamma\left(X_{f}, \mathcal{L}^{\otimes n}\right)$. It is easy to see that this morphism is injective and that the diagram

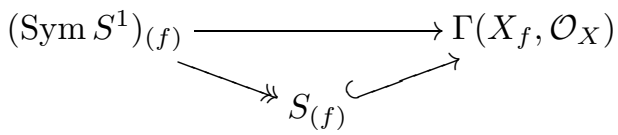

commutes. This shows that $V_{f}$ is the scheme-theoretic image of $X_{f}$ in $U_{f}$ (cf. [EGA I], (6.10)).

The flatness of $X^{c} \rightarrow Y$ is clear, since as we already remarked, all the $\mathcal{S}^{i}(i \geq 0)$ are locally free $\mathcal{O}_{Y}$-modules.

Lemma 8.2. Let $S$ be a $k$-scheme, let $\left(\mathcal{C}, \pi, s_{1}, s_{2}, h\right)$ be a modification of $\left(\tilde{C}_{0}, p_{1}, p_{2}\right)$ over $S$ (cf. 4.4). Then the canonical morphisms $\mathcal{O}_{S} \rightarrow \pi_{*} \mathcal{O}_{\mathcal{C}}$ and $\mathcal{O}_{\tilde{C} \times S} \rightarrow h_{*} \mathcal{O}_{\mathcal{C}}$ are isomorphisms.

Proof. By $\left[\mathrm{Kn}\right.$, Cor. 1.5, the sheaf $h_{*}^{\prime} \mathcal{O}_{\mathcal{C}^{\prime}}$ is locally free and commutes with any base change $S^{\prime} \rightarrow S$. It is easy to see that when restricted to any geometric fibre of the projection $\operatorname{pr}_{2}: \tilde{C}_{0} \times S \rightarrow S$, the morphism $\mathcal{O}_{\tilde{C}_{0} \times S} \rightarrow h_{*}^{\prime} \mathcal{O}_{\mathcal{C}^{\prime}}$ becomes an isomorphism. Therefore $\mathcal{O}_{\tilde{C}_{0} \times S} \rightarrow h_{*}^{\prime} \mathcal{O}_{\mathcal{C}^{\prime}}$ is an isomorphism. Clearly we have $\left(\mathrm{pr}_{2}\right)_{*} \mathcal{O}_{\tilde{C}_{0} \times S} \stackrel{\sim}{\rightarrow} \mathcal{O}_{S}$. Consequently we have $\pi_{*}^{\prime} \mathcal{O}_{\mathcal{C}^{\prime}}=\left(\mathrm{pr}_{2}\right)_{*}\left(h_{*}^{\prime} \mathcal{O}_{\mathcal{C}^{\prime}}\right) \stackrel{\sim}{\rightarrow} \mathcal{O}_{S}$.

Definition 8.3. Let $Y$ be a locally noetherian connected scheme and let $\pi: X \rightarrow Y$ be a proper flat curve over $Y$. For a locally free $\mathcal{O}_{X}$-module $\mathcal{E}$ we let $\operatorname{deg}_{X / Y} \mathcal{E}:=$ $\chi\left(\operatorname{det} \mathcal{E}_{y}\right)-\chi\left(\mathcal{O}_{X_{y}}\right)$ for some $y \in Y$, where $\mathcal{E}_{y}$ is the restriction of $\mathcal{E}$ to the fibre $X_{y}$ of $\pi$ over $y$. Note that by [EGA III], (7.9.4), this number is independent of the choice of $y$. In case $Y$ is the Spec of a field, we recover Definition 3.18,

Lemma 8.4. Let $K$ be a field and let $X / K$ be a proper curve over $K$. Let $x \in$ $X(K)$ be an ordinary double point of $X$ such that the blowing up of $X$ in $x$ is the disjoint union of two curves $X_{1}$ and $X_{2}$. Let $\mathcal{L}$ be an invertible $\mathcal{O}_{X}$-module and let $\mathcal{L}_{\nu}:=i_{\nu}^{*} \mathcal{L}$, where $i_{\nu}: X_{\nu} \rightarrow X$ is the canonical closed immersion $(\nu=1,2)$. Then the following holds:

(1) $\operatorname{deg}_{X / K} \mathcal{L}=\operatorname{deg}_{X_{1} / K} \mathcal{L}_{1}+\operatorname{deg}_{X_{2} / K} \mathcal{L}_{2}$.

(2) Assume that either $\mathcal{L}_{1}$ or $\mathcal{L}_{2}$ is generated by global sections. Then we have $H^{1}(X, \mathcal{L})=H^{1}\left(X_{1}, \mathcal{L}_{1}\right) \oplus H^{1}\left(X_{2}, \mathcal{L}_{2}\right)$.

(3) If $\mathcal{L}_{\nu}$ is generated by global sections and the canonical morphisms $\Gamma\left(X_{\nu}, \mathcal{L}_{\nu}\right)^{\otimes m} \rightarrow \Gamma\left(X_{\nu}, \mathcal{L}_{\nu}^{\otimes m}\right)$ are surjective for all $m \geq 0(\nu=1,2)$, then $\mathcal{L}$ 
is generated by global sections and the canonical morphisms $\Gamma(X, \mathcal{L})^{\otimes m} \rightarrow$ $\Gamma\left(X, \mathcal{L}^{\otimes m}\right)$ are surjective for all $m \geq 0$.

(4) If $\mathcal{L}_{\nu}$ is very ample for $\nu=1,2$, then $\mathcal{L}$ is also very ample.

Proof. The first two assertions follow easily from the following exact sequence of $\mathcal{O}_{X}$-modules:

$$
0 \longrightarrow \mathcal{L} \longrightarrow\left(i_{1}\right)_{*} \mathcal{L}_{1} \oplus\left(i_{2}\right)_{*} \mathcal{L}_{2} \longrightarrow \mathcal{L}[x] \longrightarrow 0
$$

The proof of the third assertion is entirely similar to the proof of Theorem $1.8 \mathrm{c}$ ) in $[\mathrm{Kn}]$. Recall that on a proper $K$-scheme $X$ an invertible $\mathcal{O}_{X}$-module $\mathcal{L}$ is very ample if and only if the canonical morphisms $\Gamma(X, \mathcal{L}) \rightarrow \mathcal{L}[x] \oplus \mathcal{L}[y]$ and $\Gamma(X, \mathcal{L}) \rightarrow$ $\mathcal{L}_{x} / \mathfrak{m}_{x}^{2} \mathcal{L}_{x}$ are surjective for all $x \neq y \in X$. Using this criterion the fourth assertion is easy to see.

Lemma 8.5. Let $S$ be a locally noetherian connected $k$-scheme, let $\left(\mathcal{C}^{\prime}, \pi^{\prime}, s_{1}^{\prime}, s_{2}^{\prime}, h^{\prime}\right)$ be a modification of $\left(\tilde{C}_{0}, p_{1}, p_{2}\right)$ over $S$ (cf. 4.4) and let $\mathcal{E}^{\prime}$ be a locally free $\mathcal{O}_{\mathcal{C}^{\prime-}}$ module of rank $n$ which is admissible for $\left(\mathcal{C}^{\prime}, \pi^{\prime}, s_{1}^{\prime}, s_{2}^{\prime}, h^{\prime}\right)$ (cf. 4.5). Let $\mathcal{L}_{0}$ be an

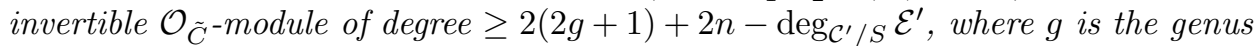
of $\tilde{C}_{0}$ and let $d \in\{0, \ldots, n\}$. Then with respect to the morphism $\pi^{\prime}$ the invertible $\mathcal{O}_{\mathcal{C}^{\prime}-\text { module }}$

$$
\mathcal{L}:=\left(\operatorname{det}^{\prime}\right)\left(-d s_{1}^{\prime}\right) \otimes_{\mathcal{O}_{\mathcal{C}^{\prime}}}\left(h^{\prime}\right)^{*}\left(\mathcal{L}_{0} \bigotimes_{k} \mathcal{O}_{S}\right)
$$

has the properties (1)-(3) listed in Proposition 8.1. Furthermore, if $Z$ is the component of a fibre $\mathcal{C}_{z}^{\prime}$ of $\pi^{\prime}$ which by $h^{\prime}$ is mapped isomorphically to $\tilde{C}_{0} \otimes_{k} \kappa(z)$, then $\left.\mathcal{L}\right|_{Z}$ is very ample.

Proof. It is clearly sufficient to consider the case where $S$ is the spectrum of an algebraically closed field $K$. Let $\mathcal{R}_{i}:=\left(h^{\prime}\right)^{-1}\left(p_{i}\right) \subset \mathcal{C}^{\prime}$ be the chain of rational curves mapped to to $p_{i}(i=1,2)$ and let $Z \subseteq \mathcal{C}^{\prime}$ be the component of $\mathcal{C}^{\prime}$ which is mapped isomorphically to $\tilde{C}_{0} \times S$. Since $\left.\mathcal{E}\right|_{\mathcal{R}_{i}}$ is strictly positive, it follows easily from $8.4(2)-(3)$ that $H^{1}\left(\mathcal{R}_{i},\left.\mathcal{L}\right|_{\mathcal{R}_{i}}\right)=0$, that $\left.\mathcal{L}\right|_{\mathcal{R}_{i}}$ is generated by global sections and that the morphisms $\Gamma\left(\mathcal{R}_{i},\left.\mathcal{L}\right|_{\mathcal{R}_{i}}\right)^{\otimes m} \rightarrow \Gamma\left(\mathcal{R}_{i},\left(\left.\mathcal{L}\right|_{\mathcal{R}_{i}}\right)^{\otimes m}\right)$ are surjective for $m \geq 0(i=1,2)$. By 8.4 it is clear therefore that it suffices to show that $H^{1}\left(Z,\left.\mathcal{L}\right|_{Z}\right)=0$, that $\left.\mathcal{L}\right|_{Z}$ is very ample and that the morphism $\Gamma\left(Z,\left.\mathcal{L}\right|_{Z}\right)^{\otimes m} \rightarrow$ $\Gamma\left(Z,\left(\left.\mathcal{L}\right|_{Z}\right)^{\otimes m}\right)$ is surjective for all $m \geq 0$. By 8.4 (1) we have $\operatorname{deg}_{Z / K}\left(\left.\mathcal{E}\right|_{Z}\right)=$ $\operatorname{deg}_{\mathcal{C}^{\prime} / K}(\mathcal{E})-\sum_{i=1}^{2} \operatorname{deg}_{\mathcal{R}_{i} / K}\left(\left.\mathcal{E}\right|_{\mathcal{R}_{i}}\right) \geq \operatorname{deg}_{\mathcal{C}^{\prime} / K}(\mathcal{E})-n$. Therefore we have

$$
\operatorname{deg}_{Z / K}\left(\left.\mathcal{L}\right|_{Z}\right) \geq \operatorname{deg}_{Z / K}\left(\left.\mathcal{E}\right|_{Z}\right)-d+\operatorname{deg}_{\tilde{C}_{0} / k} \mathcal{L}_{0} \geq 2(2 g+1)
$$

It is well known that this lower bound for the degree of $\mathcal{L}_{Z}:=\left.\mathcal{L}\right|_{Z}$ implies very ampleness and the vanishing of the first cohomology of $\mathcal{L}_{Z}$. The morphisms $\Gamma\left(Z, \mathcal{L}_{Z}\right)^{\otimes m}$ $\rightarrow \Gamma\left(Z, \mathcal{L}_{Z}^{\otimes m}\right)$ are trivially surjective for $m=0,1$. Let $\mathcal{L}_{1}, \mathcal{L}_{2}$ be two invertible $\mathcal{O}_{Z^{-}}$ modules with $\mathcal{L}_{1} \otimes \mathcal{L}_{2}=\mathcal{L}_{Z}$ and $\operatorname{deg}_{Z / K} \mathcal{L}_{i} \geq 2 g+1$ for $i=1,2$. For $m \geq 1$ consider the commutative diagram

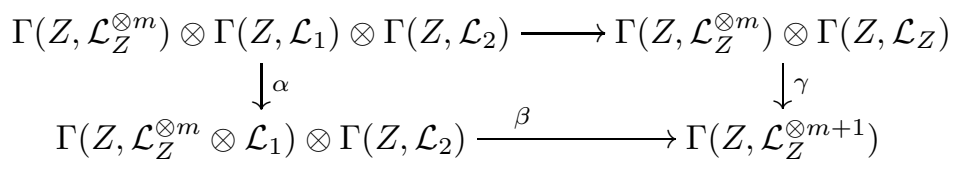

By the generalized lemma of Castelnuovo (cf. $\underline{\mathrm{Kn}}$, p.170) the vanishing of $H^{1}\left(Z, \mathcal{L}_{Z}^{\otimes m} \otimes \mathcal{L}_{1}^{-1}\right)$ and of $H^{1}\left(Z, \mathcal{L}_{Z}^{\otimes m} \otimes \mathcal{L}_{1} \otimes \mathcal{L}_{2}^{-1}\right)$ implies the surjectivity of $\alpha$ 
and $\beta$, respectively. The surjectivity of $\gamma$ now follows. By induction this shows that $\Gamma\left(Z, \mathcal{L}_{Z}\right)^{\otimes m} \rightarrow \Gamma\left(Z, \mathcal{L}_{Z}^{\otimes m}\right)$ is indeed surjective for all $m \geq 0$.

Proposition 8.6. Let $S$ be a $k$-scheme, let $\left(\mathcal{C}^{\prime}, \pi^{\prime}, s_{1}^{\prime}, s_{2}^{\prime}, h^{\prime}\right)$ be a modification of

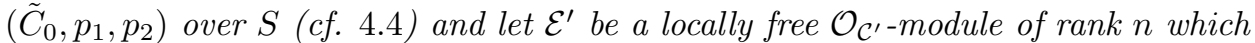
is admissible for $\left(\mathcal{C}^{\prime}, \pi^{\prime}, s_{1}^{\prime}, s_{2}^{\prime}, h^{\prime}\right)$ (cf. 4.5). Assume that $\mathcal{E}^{\prime}$ is of extremal degree $\geq\left(d_{1}, d_{2}\right)$ (cf. 4.5). There exists a modification $\left(\mathcal{C}, \pi, s_{1}, s_{2}, h\right)$ of $\left(\tilde{C}_{0}, p_{1}, p_{2}\right)$ over $S$ and an $S$-morphism $f: \mathcal{C}^{\prime} \rightarrow \mathcal{C}$ such that the following holds:

(1) $h \circ f=h^{\prime}$ and $f \circ s_{i}=s_{i}^{\prime}$ for $i=1,2$.

(2) The diagram

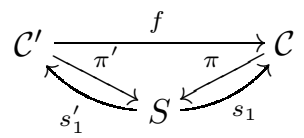

defines a simple modification of $\left(\mathcal{C}, \pi, s_{1}\right)$ in the sense of 5.1 and the bundle $\mathcal{E}^{\prime}$ is admissible of degree $d_{1}$ (in the sense of 7.1 ) for $\left(\mathcal{C}^{\prime}, f, \pi^{\prime}, s_{1}^{\prime}\right)$.

(3) Let

$$
\mathcal{E}:=\left(f_{*} \mathcal{E}^{\prime}\left(-s_{1}^{\prime}\right)\right)\left(s_{1}\right)
$$

Then $\mathcal{E}$ is a locally free $\mathcal{O}_{\mathcal{C}}$-module of rank $n$ and is admissible for $\left(\mathcal{C}, \pi, s_{1}\right.$, $\left.s_{2}, h\right)$. The extremal degree of $\mathcal{E}$ is $\geq\left(d_{1}+1, d_{2}\right)$.

Furthermore, by these properties the data $\left(\mathcal{C}, \pi, s_{1}, s_{2}, h\right)$ and $f: \mathcal{C}^{\prime} \rightarrow \mathcal{C}$ are uniquely determined in the following sense: Let $\left(\mathcal{C}^{0}, \pi^{0}, s_{1}^{0}, s_{2}^{0}, h^{0}\right)$ and $f^{0}: \mathcal{C}^{\prime} \rightarrow \mathcal{C}^{0}$ be another set of data of the same kind. Then there exists a unique isomorphism $\mathcal{C} \stackrel{\sim}{\rightarrow} \mathcal{C}^{0}$ such that the obvious diagrams commute.

Proof. We first prove the uniqueness of $\left(\mathcal{C}, \pi, s_{1}, s_{2}, h\right)$ and $f: \mathcal{C}^{\prime} \rightarrow \mathcal{C}$. Let $z \in S$. By properties 8.6 (2) and 8.6 (3) the morphism $f_{z}: \mathcal{C}_{z}^{\prime} \rightarrow \mathcal{C}_{z}$ induced by $f$ between the fibres over $z$ is not an isomorphism if and only if $\left.\mathcal{E}^{\prime}\right|_{\mathcal{C}_{z}^{\prime}}$ is of extremal degree $\left(d_{1}, d\right)$ for some $d \geq d_{2}$. The same holds for $f^{0}$, where $\left(\mathcal{C}^{0}, \pi^{0}, s_{1}^{0}, s_{2}^{0}, h^{0}\right)$ and $f^{0}: \mathcal{C}^{\prime} \rightarrow \mathcal{C}^{0}$ is another set of data satisfying 8.6(1)-8.6(3). Therefore $f^{0}$ is constant on the fibres of $f$. The morphism $f$ is proper by assumption and the morphism $\mathcal{O}_{\mathcal{C}} \rightarrow f_{*} \mathcal{O}_{\mathcal{C}^{\prime}}$ is an isomorphism by 5.9. Therefore we may apply EGA II], (8.11.1), to conclude that there is a unique morphism $u: \mathcal{C} \rightarrow \mathcal{C}^{0}$ such that $f^{0}=u \circ f$. With the same argument, there exists a unique morphism $v: \mathcal{C}^{0} \rightarrow \mathcal{C}$ with $f=v \circ f^{0}$ and we have $u \circ v=\mathrm{id}_{\mathcal{C}^{0}}$ and $v \circ u=\mathrm{id}_{\mathcal{C}}$. In particular, $u$ is an isomorphism. It is clear that $u \circ s_{i}=s_{i}^{0}$ for $i=1,2$ and it follows immediately that $\pi=\pi^{0} \circ u$. Since the morphism $h$ is clearly constant on the fibres of $h^{0} \circ u$ and furthermore the morphism $h^{0}$ is proper by assumption and we have $h_{*}^{0} \mathcal{O}_{\mathcal{C}^{0}}=\mathcal{O}_{\tilde{C} \times S}$ by 8.2 , we may again apply [EGA II], (8.11.1), to conclude that there is a unique morphism $w: \tilde{C} \times S \rightarrow \tilde{C} \times S$ such that $h=w \circ h^{0} \circ u$. Then we have $h^{\prime}=h \circ f=w \circ h^{0} \circ u \circ f=w \circ h^{\prime}$ and yet another application of EGA II], (8.11.1), leads to the conclusion that $w$ is in fact the identitiy on $\tilde{C} \times S$, in particular that $h=h^{0} \circ u$. This proves the uniqueness statement.

For the existence it suffices by 4.6 to consider the case where $S$ is locally noetherian. Furthermore we may assume $S$ to be connected. By 8.5 there is a very ample invertible $\mathcal{O}_{\tilde{C}_{0}}$-module $\mathcal{L}_{0}$ such that the $\mathcal{O}_{\mathcal{C}^{\prime}}$-module

$$
\mathcal{L}:=\left(\operatorname{det} \mathcal{E}^{\prime}\right)\left(-d_{1} s_{1}^{\prime}\right) \otimes_{\mathcal{O}_{\mathcal{C}^{\prime}}}\left(h^{\prime}\right)^{*}\left(\mathcal{L}_{0} \otimes_{k} \mathcal{O}_{S}\right)
$$


has the properties (1)-(3) listed in 8.1 with respect to $\pi^{\prime}$. Let $\mathcal{C} \subset \operatorname{Proj}\left(\operatorname{Sym} \pi_{*}^{\prime} \mathcal{L}\right)$ be the scheme-theoretic image of the morphism $\mathcal{C}^{\prime} \rightarrow \operatorname{Proj}\left(\operatorname{Sym} \pi_{*}^{\prime} \mathcal{L}\right)$ induced by the surjection $\left(\pi^{\prime}\right)^{*} \pi_{*}^{\prime} \mathcal{L} \rightarrow \mathcal{L}$. We have the commutative diagram

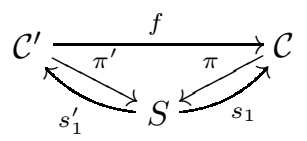

where $\pi: \mathcal{C} \rightarrow S$ is the structure morphism and $s_{i}:=f \circ s_{i}^{\prime}$ for $i=1,2$. Since by 8.2 we have $\pi_{*}^{\prime} \mathcal{O}_{\mathcal{C}^{\prime}}=\mathcal{O}_{S}$, we can apply Proposition 8.1 to conclude that $\pi$ is a flat morphism. Since $\pi$ and $\pi^{\prime}$ are obviously proper, the morphism $f$ is also proper. Let $z \in S$ and let $\mathcal{C}_{z}^{\prime}, \mathcal{C}_{z}, f_{z}$ etc. denote the objects over $\operatorname{Spec} \kappa(z)$ induced by pull back. By definition, the curve $\mathcal{C}_{z}^{\prime}$ is of the following shape:

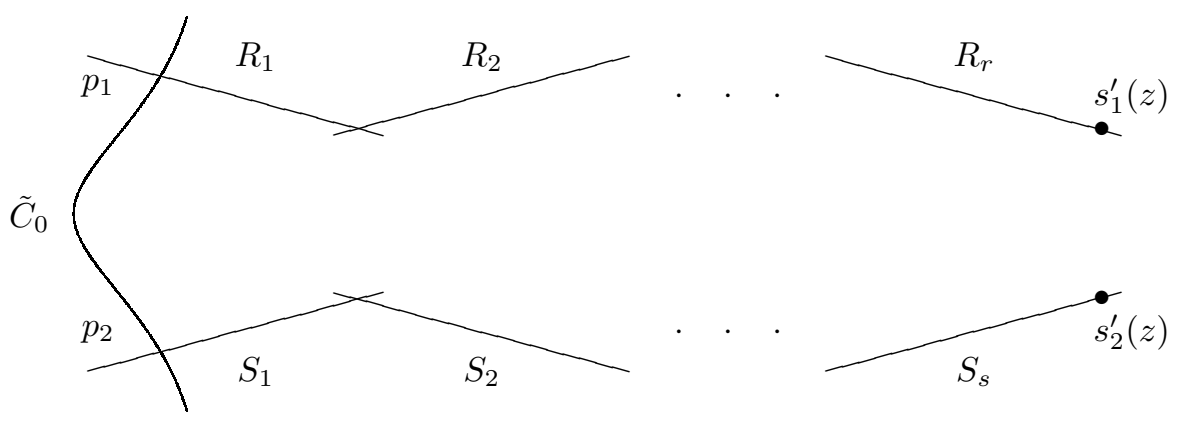

for some $r, s \geq 0$, with $R_{i} \cong S_{j} \cong \mathbb{P}_{\kappa(z)}^{1}$. Let $D \subseteq \mathcal{C}_{z}^{\prime}$ be the closed subscheme of $\mathcal{C}_{z}^{\prime}$ consisting of all components except $R_{r}$. It is not difficult to check that $\left.\mathcal{L}_{z}\right|_{D}$ is very ample and that $\mathcal{L}_{z}$ is very ample if $d_{1}<\left.\operatorname{deg} \mathcal{E}_{z}^{\prime}\right|_{R_{r}}=: d$. Furthermore it is clear that $\left.\mathcal{L}_{z}\right|_{R_{r}}$ is trivial if $d_{1}=d$. It follows that there are two possible cases:

(1) $d_{1}<d$. Then $f_{z}: \mathcal{C}_{z}^{\prime} \rightarrow \mathcal{C}_{z}$ is an isomorphism.

(2) $d_{1}=d$. Then $\mathcal{C}_{z} \cong D$ and $f_{z}$ is the contraction of the rational curve $R_{r}$.

It is now clear that $\mathcal{C}$ is a flat curve over $S$, that the $s_{i}$ are sections of $\pi$ which meet $\mathcal{C}$ in points where $\pi$ is smooth, that the diagram $(*)$ defines a simple modification of $\left(\mathcal{C}, \pi, s_{1}\right)$ and that $\mathcal{E}^{\prime}$ is admissible of degree $d_{1}$ for $\left(\mathcal{C}^{\prime}, f, \pi^{\prime}, s_{1}^{\prime}\right)$.

In particular, the morphism $h^{\prime}$ is constant on the fibres of $f$. Since $f$ is proper and we have $f_{*} \mathcal{O}_{\mathcal{C}^{\prime}}=\mathcal{O}_{\mathcal{C}}$ by [5.9, we can apply [EGA II], (8.11.1), to show that there exists a unique morphism $h: \mathcal{C} \rightarrow \tilde{C}_{0} \times S$ with $h^{\prime}=h \circ f$. It is clear from the fibrewise consideration above that $\left(\mathcal{C}, \pi, s_{1}, s_{2}, h\right)$ is a modification of $\left(\tilde{C}_{0}, p_{1}, p_{2}\right)$.

That $\mathcal{E}:=\left(f_{*} \mathcal{O}_{\mathcal{C}^{\prime}}\left(-s_{1}^{\prime}\right)\right)\left(s_{1}\right)$ is locally free of rank $n$, follows from 7.6 and 7.5 , For the remaining part of property (3) it suffices to consider the case $S=\operatorname{Spec} K$ for some field $K$. Let $R^{\prime} \subset \mathcal{C}^{\prime}$ be the fibre $f^{-1}\left(s_{1}\right)$. Since otherewise $f$ is an isomorphism, we may assume that $R^{\prime}$ is a chain of rational curves of length $r \geq 1$. Let $R_{i}(i=1, \ldots, r)$ be its successive irreducible components. For $i=1, \ldots, r-1$ let $x_{i} \in R^{\prime}(K)$ be the point where $R_{i}$ and $R_{i+1}$ intersect. Let $x_{r}:=s_{1}^{\prime} \in R_{r}(K)$ and let $x_{0}:=p_{1} \in R_{1}(K)$ be the point, where $R^{\prime}$ meets the rest of $\mathcal{C}^{\prime}$. We identify $\mathcal{C}$ with the closed subset of $\mathcal{C}^{\prime}$ consisting of the union of all irreducible components of $\mathcal{C}^{\prime}$, except $R_{r}$. Therefore we consider $R:=\bigcup_{i=1}^{r-1} R_{i}$ also as a closed subscheme of $\mathcal{C}$ and we have $x_{r-1}=s_{1}$. For $i=1, \ldots, r$ (resp. $\left.i=1, \ldots, r-1\right)$ let $\mathcal{E}_{i}^{\prime}:=\left.\mathcal{E}^{\prime}\right|_{R_{i}}$ and 
$\delta_{i}^{\prime}:=\operatorname{deg}\left(\mathcal{E}_{i}^{\prime}\right)\left(\operatorname{resp} . \mathcal{E}_{i}:=\left.\mathcal{E}\right|_{R_{i}}\right.$ and $\left.\delta_{i}:=\operatorname{deg}\left(\mathcal{E}_{i}\right)\right)$. Since $\left.\mathcal{E}^{\prime}\right|_{R^{\prime}}$ is strictly standard, we have canonical exact sequences

$$
0 \rightarrow \mathcal{F}_{i}^{\prime} \rightarrow \mathcal{E}_{i}^{\prime} \rightarrow \mathcal{G}_{i}^{\prime} \rightarrow 0
$$

for all $i=1, \ldots, r$, where $\mathcal{F}_{i}^{\prime} \cong \bigoplus^{\delta_{i}^{\prime}} \mathcal{O}_{R_{i}}(1)$ and $\mathcal{G}_{i}^{\prime} \cong \bigoplus^{n-\delta_{i}^{\prime}} \mathcal{O}_{R_{i}}$. It is easy to see that $f_{*} \mathcal{E}^{\prime}\left(-s_{1}^{\prime}\right)=\operatorname{ker}\left(\mathcal{E}^{\prime} \rightarrow \mathcal{E}^{\prime}\left[x_{r-1}\right] \rightarrow \mathcal{G}_{r}\left[x_{r-1}\right]\right)$. Therefore we have an exact diagram of $\mathcal{O}_{R_{r-1}}$-modules as follows:

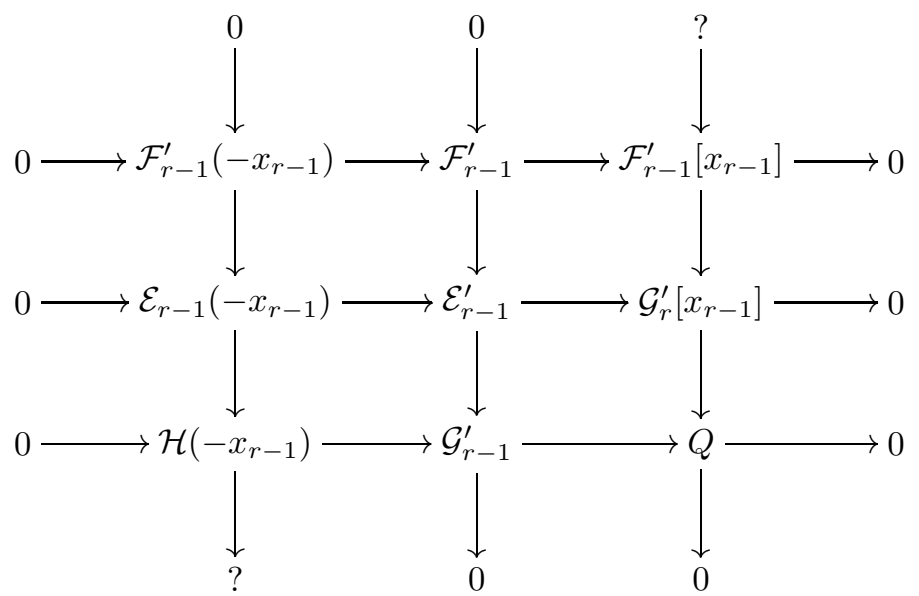

where $Q:=\operatorname{coker}\left(\mathcal{F}_{r-1}^{\prime}\left[x_{r-1}\right] \rightarrow \mathcal{G}_{r}^{\prime}\left[x_{r-1}\right]\right)$ and $\mathcal{H}:=\operatorname{ker}\left(\mathcal{G}_{r-1}^{\prime} \rightarrow Q\right)\left(x_{r-1}\right)$. I claim that this diagram remains exact if we replace "?" by zero. Indeed, we have

$$
\begin{gathered}
\operatorname{ker}\left(\mathcal{F}_{r-1}^{\prime}\left[x_{r-1}\right] \rightarrow \mathcal{G}_{r}^{\prime}\left[x_{r-1}\right]\right)=\mathcal{F}_{r-1}^{\prime}\left[x_{r-1}\right] \cap \mathcal{F}_{r}^{\prime}\left[x_{r-1}\right] \\
\quad=H^{0}\left(R_{r-1} \cup R_{r},\left.\mathcal{E}^{\prime}\right|_{R_{r-1} \cup R_{r}}\left(-x_{r-2}-x_{r}\right)\right)
\end{gathered}
$$

which is zero, since $\mathcal{E}^{\prime}$ and therefore also $\left.\mathcal{E}^{\prime}\right|_{R_{r-1} \cup R_{r}}$ is admissible. By definition of $\mathcal{H}$ it is clear that it is strictly standard of degree $\mathrm{rk}\left(\mathcal{G}_{r-1}^{\prime}\right)-\operatorname{dim}(Q)=\delta_{r}^{\prime}$. Being an extension of $\mathcal{F}_{r-1}^{\prime}$ and $\mathcal{H}$, the $\mathcal{O}_{R_{r-1}}$-module $\mathcal{E}_{r-1}$ is therefore strictly standard of degree $\delta_{r-1}=\delta_{r}^{\prime}+\delta_{r-1}^{\prime}$. Furthermore, we have

$$
H^{0}\left(R,\left.\mathcal{E}\right|_{R}\left(-x_{0}-x_{r-1}\right)\right)=H^{0}\left(R,\left.f_{*} \mathcal{E}^{\prime}\right|_{R^{\prime}}\left(-x_{0}-x_{r}\right)\right)=(0) \quad .
$$

This proves the admissibility of $\mathcal{E}$

\section{Gieseker VeCtor Bundle Data AND Generalized isomorphisms}

Let $\operatorname{VB}\left(\tilde{C}_{0}\right)$ be the moduli stack of vector bundles of rank $n$ over $\tilde{C}_{0}$ (cf. [LM], (4.6.2.1)). Let $\mathcal{E}_{\text {univ }}$ be the universal bundle over $\tilde{C}_{0} \times \operatorname{VB}\left(\tilde{C}_{0}\right)$. By abuse of notation, we also denote by $p_{i}$ the section of the projection $\tilde{C}_{0} \times \operatorname{VB}\left(\tilde{C}_{0}\right) \rightarrow \operatorname{VB}\left(\tilde{C}_{0}\right)$ which is induced by the point $p_{i} \in \tilde{C}_{0}(i=1,2)$. The purpose of this section is to show that the stack $\operatorname{GVBD}\left(\tilde{C}_{0}, p_{1}, p_{2}\right)$ is canonically isomorphic to the stack $\mathrm{KGl}_{n}\left(p_{1}^{*} \mathcal{E}_{\text {univ }}, p_{2}^{*} \mathcal{E}_{\text {univ }}\right)$. By definition, an $S$-valued point of $\mathrm{KGl}_{n}\left(p_{1}^{*} \mathcal{E}_{\text {univ }}, p_{2}^{*} \mathcal{E}_{\text {univ }}\right)$ is a vector bundle $\mathcal{E}$ over $C_{0} \times S$ together with a generalized isomorphism between the $\mathcal{O}_{S}$-modules $\left.\mathcal{E}\right|_{\left\{p_{1}\right\} \times S}$ and $\left.\mathcal{E}\right|_{\left\{p_{2}\right\} \times S}($ cf. [K1] $)$.

Construction 9.1. Let $S$ be a $k$-scheme and let $\left(\mathcal{C}, \pi, s_{1}, s_{2}, h ; \mathcal{E}, \varphi\right)$ an object in $\operatorname{GVBD}_{n}\left(\tilde{C}_{0}, p_{1}, p_{2}\right)(S)$ (cf. Definition 4.7). We want to construct a vector bundle $h_{\bullet}(\mathcal{E})$ of rank $n$ on $\tilde{C}_{0} \times{ }_{\operatorname{Spec}(k)} S$ out of this data. 
By setting

$$
\left(\mathcal{C}^{(n)}, \pi^{(n)}, s_{1}^{(n)}, s_{2}^{(n)}, h^{(n)}\right):=\left(\mathcal{C}, \pi, s_{1}, s_{2}, h\right)
$$

and $\mathcal{E}^{(n)}:=\mathcal{E}$ and repeatedly applying 8.6 , we obtain a sequence

$$
\left(\mathcal{C}^{(i)}, \pi^{(i)}, s_{1}^{(i)}, s_{2}^{(i)}, h^{(i)}\right)_{i=0, \ldots, n}
$$

of modifications of $\left(\tilde{C}_{0}, p_{1}, p_{2}\right)$ over $S$, which are linked by morphisms as follows

$$
\mathcal{C}=\mathcal{C}^{(n)} \stackrel{f^{(n)}}{\longrightarrow} \mathcal{C}^{(n-1)} \stackrel{f^{(n-1)}}{\longrightarrow} \ldots \stackrel{f^{(1)}}{\longrightarrow} \mathcal{C}^{(0)}
$$

and for each $i \in[0, n]$ a locally free $\mathcal{O}_{\mathcal{C}^{(i)}}$-module $\mathcal{E}^{(i)}$ of rank $n$, which is admissible for $\left(\mathcal{C}^{(i)}, \pi^{(i)}, s_{1}^{(i)}, s_{2}^{(i)}, h^{(i)}\right)$ and which is of extremal degree $\geq(n-i+1,1)$.

Now we set

$$
\left(\mathcal{C}^{[n]}, \pi^{[n]}, s_{1}^{[n]}, s_{2}^{[n]}, h^{[n]}\right):=\left(\mathcal{C}^{(0)}, \pi^{(0)}, s_{1}^{(0)}, s_{2}^{(0)}, h^{(0)}\right)
$$

and $\mathcal{E}^{[n]}:=\mathcal{E}^{(0)}$ and again successively apply 8.6 with the role of $s_{1}$ and $s_{2}$ interchanged, thus obtaining a sequence

$$
\left(\mathcal{C}^{[i]}, \pi^{[i]}, s_{1}^{[i]}, s_{2}^{[i]}, h^{[i]}\right)_{i=0, \ldots, n}
$$

of modifications of $\left(\tilde{C_{0}}, p_{1}, p_{2}\right)$ over $S$, a chain

$$
\mathcal{C}^{(0)}=\mathcal{C}^{[n]} \stackrel{f^{[n]}}{\longrightarrow} \mathcal{C}^{[n-1]} \stackrel{f^{[n-1]}}{\longrightarrow} \ldots \stackrel{f^{[1]}}{\longrightarrow} \mathcal{C}^{[0]}
$$

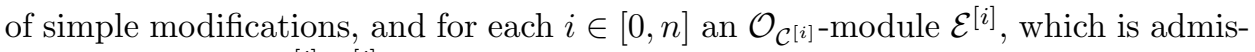
sible for $\left(\mathcal{C}^{[i]}, \pi^{[i]}, s_{1}^{[i]}, s_{2}^{[i]}, h^{[i]}\right)$ and of extremal degree $\geq(n+1, n-i+1)$.

In particular, $\mathcal{E}^{[0]}$ is of extremal degree $\geq(n+1, n+1)$ which is easily seen to imply that the morphism $h^{[0]}: \mathcal{C}^{[0]} \rightarrow \tilde{C}_{0} \times_{\operatorname{Spec}(k)} S$ is an isomorphism. We set $h_{\bullet}(\mathcal{E}):=h_{*}^{[0]}\left(\mathcal{E}^{[0]}\right)$.

Construction 9.2. Let $S$ be a $k$-scheme, let $\left(\mathcal{C}, \pi, s_{1}, s_{2}, h ; \mathcal{E}, \varphi\right)$ be an object in $\operatorname{GVBD}_{n}\left(\tilde{C}_{0}, p_{1}, p_{2}\right)(S)$ (cf. Definition 4.7) and let $h \bullet(\mathcal{E})$ be the $\mathcal{O}_{\tilde{C}_{0} \times \text { Spec }(k)} S^{\text {-module }}$ associated to this data (cf. 9.1). We want to construct a generalized isomorphism

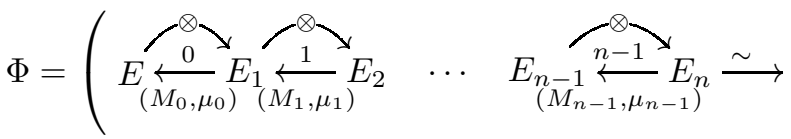

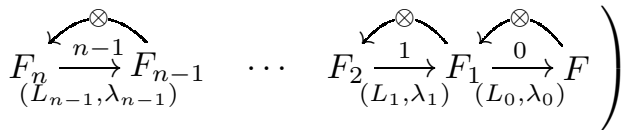

(cf. [K1]) between the two $\mathcal{O}_{S}$-modules $E:=p_{1}^{*} h_{\bullet}(\mathcal{E})$ and $F:=p_{2}^{*} h_{\bullet}(\mathcal{E})$, where by abuse of notation we write $p_{i}: S \rightarrow \tilde{C}_{0} \times \operatorname{Spec}(k) S$ for the section of $\tilde{C}_{0} \times \operatorname{Spec}(k) S \rightarrow S$ induced by the point $p_{i} \in \tilde{C}_{0}(k)$.

Let

$$
E_{i}:=\left(s_{1}^{(i)}\right)^{*} \mathcal{E}^{(i)} \quad, \quad F_{i}:=\left(s_{2}^{[i]}\right)^{*} \mathcal{E}^{[i]}
$$

for $i=0, \ldots, n$ and for $i=1, \ldots, n$ let

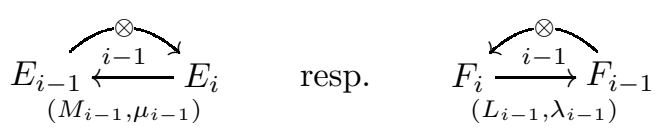


be the bf-morphism associated by Construction 7.2 to the simple modification

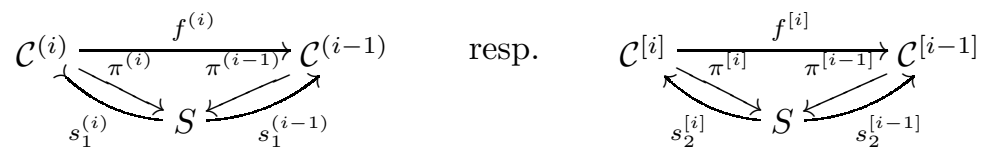

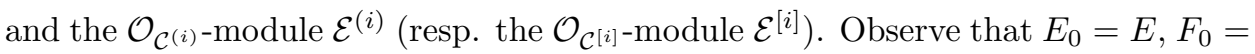
$F, E_{n}=s_{1}^{*} \mathcal{E}$ and $F_{n}=s_{2}^{*} \mathcal{E}$. In particular, $\varphi$ defines an isomorphism $E_{n} \stackrel{\sim}{\rightarrow} F_{n}$ and thus we have constructed all the data $\Phi$, which make up a generalized isomorphism from $E$ to $F$.

Lemma 9.3. The data

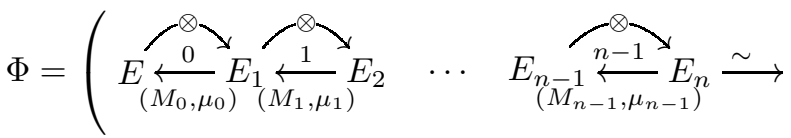

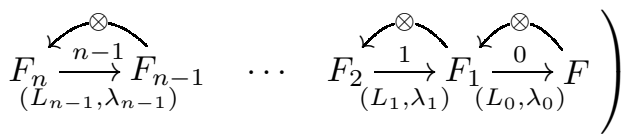

constructed in 9.2 is a generalized isomorphism from $E$ to $F$. The association

$$
\left(\mathcal{C}, \pi, s_{1}, s_{2}, h ; \mathcal{E}, \varphi\right) \mapsto\left(h_{\bullet} \mathcal{E}, \Phi\right)
$$

is functorial with respect to isomorphisms and commutes with base-change $S^{\prime} \rightarrow S$, and hence defines a morphism

$$
\operatorname{GVBD}\left(\tilde{C}_{0}, p_{1}, p_{2}\right) \longrightarrow \operatorname{KGl}\left(p_{1}^{*} \mathcal{E}_{\text {univ }}, p_{2}^{*} \mathcal{E}_{\text {univ }}\right)
$$

of k-groupoids.

Proof. By construction it is clear that the association $\left(\mathcal{C}, \pi, s_{1}, s_{2}, h ; \mathcal{E}, \varphi\right) \mapsto(h \bullet \mathcal{E}, \Phi)$ is functorial with respect to isomorphisms and commutes with base change. All that remains to be shown is that $\Phi$ satisfies properties 1 and 2 in [K1, Definition 5.2. Since these are pointwise properties, we may assume that $S=\operatorname{Spec}(K)$ for some field $K$. Consider the following situation:
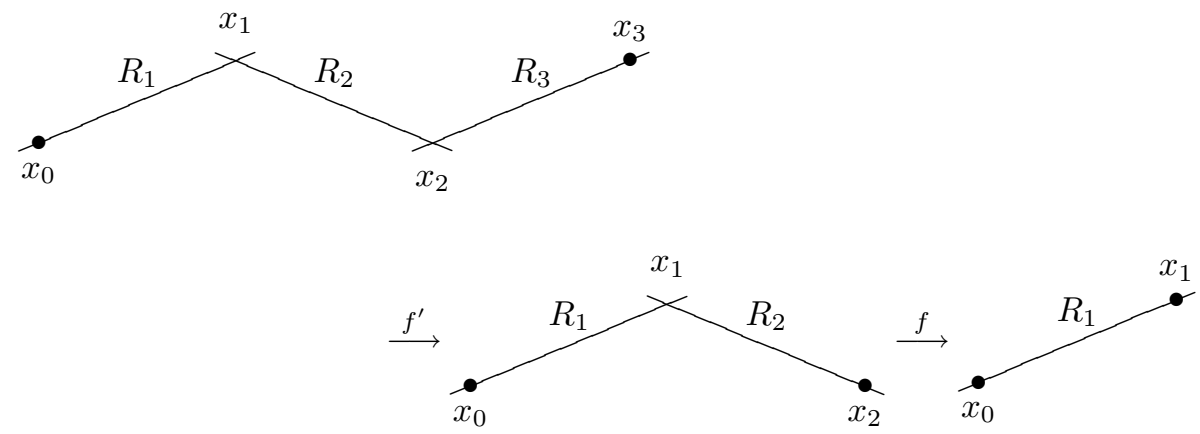

where the $R_{i}$ are projective lines over $K$ and $f^{\prime}: R^{\prime \prime}:=\bigcup_{i=1}^{3} R_{i} \rightarrow R^{\prime}:=\bigcup_{i=1}^{2} R_{i}$ and $f: R^{\prime} \rightarrow R:=R_{1}$ are simple modifications over $K$ as indicated. Let $\mathcal{E}^{\prime \prime}$ be an admissible $\mathcal{O}_{R^{\prime \prime}}$-module of rank $n$, let $\mathcal{E}^{\prime}:=\left(f_{*}^{\prime} \mathcal{E}^{\prime \prime}\left(-x_{3}\right)\right)\left(x_{2}\right)$ and let $\mathcal{E}:=$ $\left(f_{*} \mathcal{E}^{\prime}\left(-x_{2}\right)\right)\left(x_{1}\right)$. Denote by $\mathcal{E}_{i}^{\prime \prime}\left(\right.$ resp. $\left.\mathcal{E}_{i}^{\prime}, \mathcal{E}_{i}\right)$ the restriction of $\mathcal{E}^{\prime \prime}\left(\right.$ resp. of $\left.\mathcal{E}^{\prime}, \mathcal{E}\right)$ to the component $R_{i}$. We have canonical exact sequences

$$
0 \longrightarrow \mathcal{F}_{i}^{\prime \prime} \longrightarrow \mathcal{E}_{i}^{\prime \prime} \longrightarrow \mathcal{G}_{i}^{\prime \prime} \longrightarrow 0
$$


of $\mathcal{O}_{R_{i}}$-modules, where $\mathcal{G}_{i}^{\prime \prime}$ is trivial and $\mathcal{F}_{i}^{\prime \prime}$ is a direct sum of copies of $\mathcal{O}_{R_{i}}(1)$. Analogously let $\mathcal{F}_{i}^{\prime}$ and $\mathcal{G}_{i}^{\prime}$ be the canonical subsheaf and quotient sheaf of $\mathcal{E}_{i}^{\prime}$. By 7.3 we have canonical diagrams

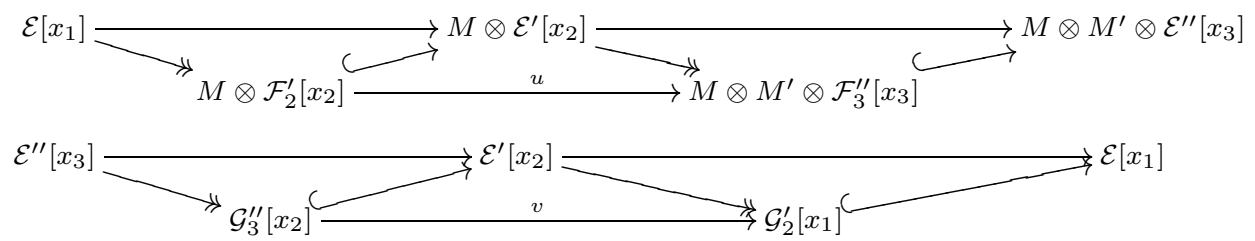

where $M:=\left(\mathfrak{m}_{2} / \mathfrak{m}_{2}^{2}\right) \otimes\left(\mathfrak{m}_{1} / \mathfrak{m}_{1}^{2}\right)^{\vee}, M^{\prime}:=\left(\mathfrak{m}_{3} / \mathfrak{m}_{3}^{2}\right) \otimes\left(\mathfrak{m}_{2} / \mathfrak{m}_{2}^{2}\right)^{\vee}$ and $\mathfrak{m}_{i}$ is the maximal ideal of $\mathcal{O}_{R_{i}, x_{i}}$. To show that $\Phi$ satisfies property 1 in [K1], Definition 5.2 , it is clearly sufficient to show that the morphisms $u$ and $v$ in these diagrams are surjective. In fact by the exact diagram

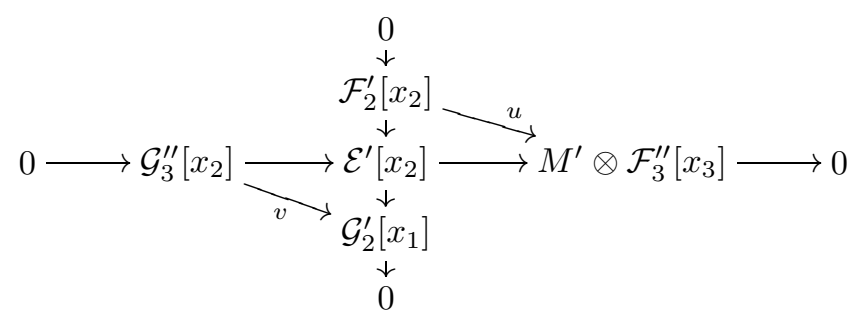

it suffices to prove surjectivity of either $u$ or $v$. From the canonical exact sequence of $\mathcal{O}_{R_{2}}$-modules (cf. 7.3)

$$
0 \longrightarrow \mathcal{E}_{2}^{\prime}\left(-x_{2}\right) \longrightarrow \mathcal{E}_{2}^{\prime \prime} \longrightarrow \mathcal{G}_{3}^{\prime \prime}\left[x_{2}\right] \longrightarrow 0
$$

we deduce the exact diagram of $K$-vector spaces:

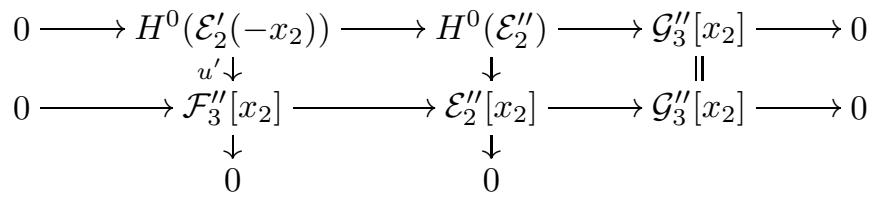

Observe that we have canonical isomorphisms

$$
H^{0}\left(\mathcal{E}_{2}^{\prime}\left(-x_{2}\right)\right)=H^{0}\left(\mathcal{F}_{2}^{\prime}\left(-x_{2}\right)\right)=\left(\mathfrak{m}_{2} / \mathfrak{m}_{2}^{2}\right) \otimes \mathcal{F}_{2}^{\prime}\left[x_{2}\right]
$$

and

and that the morphism

$$
\mathcal{F}_{3}^{\prime \prime}\left[x_{2}\right]=\left(\mathfrak{m}_{3} / \mathfrak{m}_{3}^{2}\right) \otimes \mathcal{F}_{3}^{\prime \prime}\left[x_{3}\right]
$$

$$
\left(\mathfrak{m}_{2} / \mathfrak{m}_{2}^{2}\right) \otimes \mathcal{F}_{2}^{\prime}\left[x_{2}\right]=H^{0}\left(\mathcal{E}_{2}^{\prime}\left(-x_{2}\right)\right) \stackrel{u^{\prime}}{\longrightarrow} \mathcal{F}_{3}^{\prime \prime}\left[x_{2}\right]=\left(\mathfrak{m}_{3} / \mathfrak{m}_{3}^{2}\right) \otimes \mathcal{F}_{3}^{\prime \prime}\left[x_{3}\right]
$$

coincides with the morphism $u$. This proves the surjectivity of $u$ (and of $v$ ).

Now we want to show that $\Phi$ satisfies property 2. in [K1, Definition 5.2, i.e. that $\varphi\left(\operatorname{ker}\left(E_{0} \leftarrow E_{n}\right)\right) \cap \operatorname{ker}\left(F_{n} \rightarrow F_{0}\right)=(0)$. Let $R$ (resp. $R^{\prime}$ ) be the chain of projective lines in $\mathcal{C}$, which by $h$ is contracted to the point $p_{1}$ (resp. to $p_{2}$ ) and let $r$ (resp. $r^{\prime}$ ) be its length. If $r \geq 1$ (resp. $r^{\prime} \geq 1$ ), we denote by $V_{r} \subseteq \mathcal{E}\left[s_{1}\right]$ (resp. by $\left.V_{r^{\prime}}^{\prime} \subseteq \mathcal{E}\left[s_{2}\right]\right)$ the image of $H^{0}\left(\left.\mathcal{E}\right|_{R}\left(-p_{1}\right)\right)$ in $\mathcal{E}\left[s_{1}\right]=E_{n}$ (resp. the image of $H^{0}\left(\left.\mathcal{E}\right|_{R^{\prime}}\left(-p_{2}\right)\right)$ in $\left.\mathcal{E}\left[s_{2}\right]=F_{n}\right)$. If $r=0\left(\right.$ resp. $r^{\prime}=0$ ), we set $V_{r}:=(0)$ (resp. $V_{r^{\prime}}^{\prime}=(0)$ ). Admissibility of $\mathcal{E}$ implies that $\varphi\left(V_{r}\right) \cap V_{r^{\prime}}^{\prime}=(0)$. Therefore (and by reasons of symmetry) it suffices to show the equality $\operatorname{ker}\left(E_{0} \leftarrow E_{n}\right)=V_{r}$. We 
will prove this by induction on $r$. The case $r=0$ is trivial. If $r=1$, we have $\operatorname{ker}\left(E_{0} \leftarrow E_{n}\right)=\mathcal{F}\left[s_{1}\right]=V_{1}$, where $\left.\mathcal{F} \hookrightarrow \mathcal{E}\right|_{R}$ is the canonical subsheaf of $\left.\mathcal{E}\right|_{R}$. Let $r \geq 2$ and let $f: R \rightarrow \bar{R}$ be the simple modification of $\left(R, s_{1}\right)$ which contracts the component $R_{r}$ of $R$ to a point $x_{r-1} \in \bar{R}$. As usual, we identify $\bar{R}$ with a subchain of $R$. Let $\mathcal{G}$ be the canonical quotient sheaf of $\left.\mathcal{E}\right|_{R_{r}}$ and let $\overline{\mathcal{E}}:=\left(\left.f_{*} \mathcal{E}\right|_{R}\left(-s_{1}\right)\right)\left(x_{r-1}\right)$. By 7.3 the morphism $E_{n} \rightarrow E_{0}$ factorizes as follows: $E_{n}=\mathcal{E}\left[s_{1}\right] \rightarrow \mathcal{G}\left[x_{r-1}\right] \rightarrow$ $\overline{\mathcal{E}}\left[x_{r-1}\right] \rightarrow E_{0}$. Let $\bar{V}_{r-1} \subseteq \overline{\mathcal{E}}\left[x_{r-1}\right]$ (resp. $V_{r-1} \subseteq \mathcal{E}\left[x_{r-1}\right]$ ) be the image of the map $H^{0}\left(\left.\overline{\mathcal{E}}\right|_{\bar{R}}\left(-p_{1}\right)\right) \rightarrow \overline{\mathcal{E}}\left[x_{r-1}\right]$ (resp. of the map $\left.H^{0}\left(\left.\mathcal{E}\right|_{\bar{R}}\left(-p_{1}\right)\right) \rightarrow \mathcal{E}\left[x_{r-1}\right]\right)$. By the induction hypothesis, we have $\bar{V}_{r-1}=\operatorname{ker}\left(\overline{\mathcal{E}}\left[x_{r-1}\right] \rightarrow E_{0}\right)$ and by lemma 3.3 the subspace $V_{r}$ is the preimage under $\mathcal{E}\left[s_{1}\right] \rightarrow \mathcal{G}\left[x_{r-1}\right]$ of the image of $V_{r-1}$ by $\mathcal{E}\left[x_{r-1}\right] \rightarrow \mathcal{G}\left[x_{r-1}\right]$. Therefore we have to show the following equality of subspaces of $\mathcal{G}\left[x_{r-1}\right]$ :

$$
\begin{aligned}
\bar{U} & :=\left(\begin{array}{l}
\text { preimage of } \bar{V}_{r-1} \text { under the } \\
\text { morphism } \mathcal{G}\left[x_{r-1}\right] \rightarrow \overline{\mathcal{E}}\left[x_{r-1}\right]
\end{array}\right) \\
& =\left(\begin{array}{l}
\text { image of } V_{r-1} \text { by the } \\
\text { morphism } \mathcal{E}\left[x_{r-1}\right] \rightarrow \mathcal{G}\left[x_{r-1}\right]
\end{array}\right)=: U .
\end{aligned}
$$

Consider the exact diagram of $\mathcal{O}_{\bar{R}}$-modules (cf. 7.3 ): 2

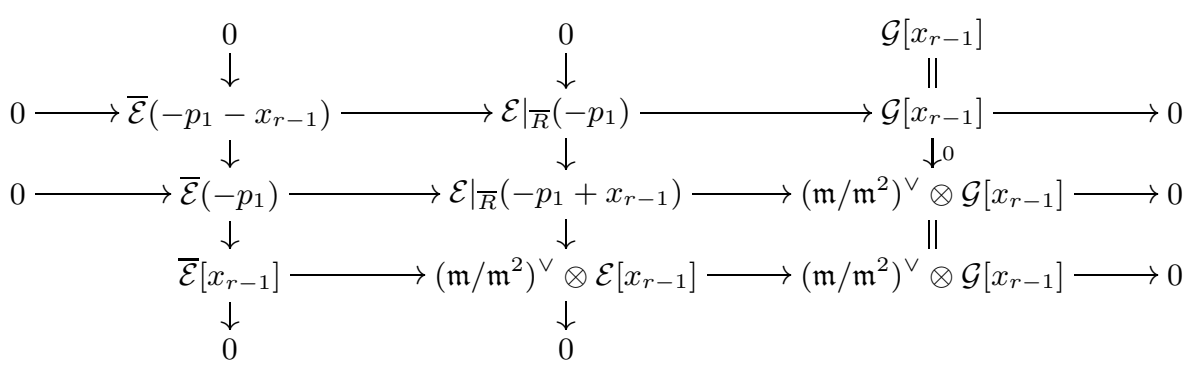

Application of $H^{0}=H^{0}(\bar{R}, \cdot)$ leads to the exact diagram of $K$-vector spaces

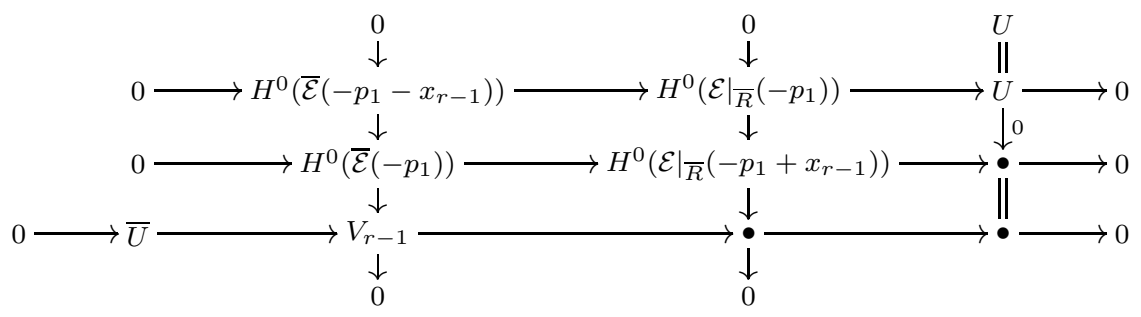

which proves $\bar{U}=U$ as required.

Remark 9.4. In 9.1, 9.2 and 9.3 we have shown that starting with a Gieseker vector bundle data, one can produce a sequence of simple modifications together with admissible bundles on them, and that the corresponding (via 7.4) bf-morphisms make up a generalized isomorphism. The following picture may help to keep track 
of which simple modification corresponds to which bf-morphism:

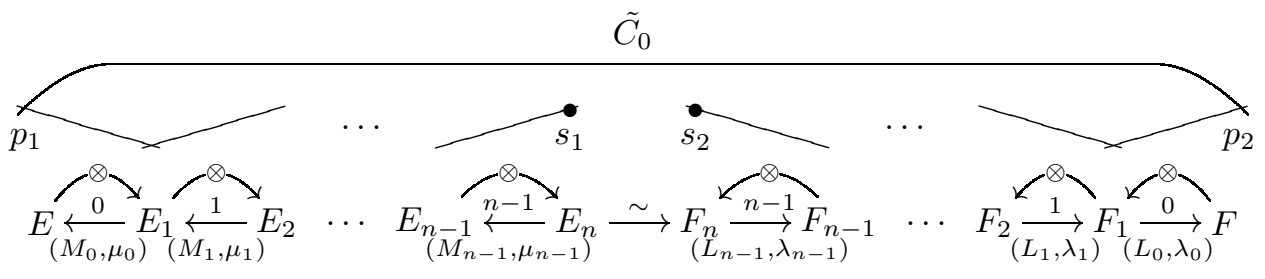

But one should keep in mind that a projective line corresponds only to those bf-morphisms, where the section $\mu_{i}$ (resp. $\lambda_{i}$ ) vanishes! In this sense the picture is slightly misleading.

Theorem 9.5. The morphism

$$
\operatorname{GVBD}\left(\tilde{C}_{0}, p_{1}, p_{2}\right) \longrightarrow \operatorname{KGl}\left(p_{1}^{*} \mathcal{E}_{\text {univ }}, p_{2}^{*} \mathcal{E}_{\text {univ }}\right)
$$

defined by Construction 9.2 is an isomorphism of algebraic k-stacks.

The proof of Theorem 9.5 will be given after Lemma 9.7 below.

Construction 9.6. Let $S$ be a $k$-scheme, let $\mathcal{F}$ be a locally free $\mathcal{O}_{\tilde{C}_{0} \times \times_{\text {Spec }(k)} S^{-}}$ module of rank $n$ and let

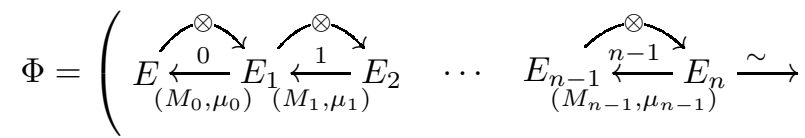

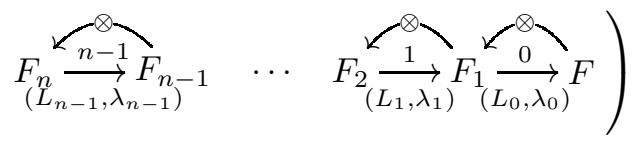

be a generalized isomorphism from $E=E_{0}:=p_{1}^{*} \mathcal{F}$ to $F=F_{0}:=p_{2}^{*} \mathcal{F}$. We want to construct a Gieseker vector bundle data $\left(\mathcal{C}, \pi, s_{1}, s_{2}, h ; \mathcal{E}, \varphi\right)$ on $\left(\tilde{C}_{0}, p_{1}, p_{2}\right)$ over $S$ (cf. 4.7).

Let

$$
\left(\mathcal{C}^{[0]}, \pi^{[0]}, s_{1}^{[0]}, s_{2}^{[0]}, h^{[0]}\right):=\left(\tilde{C}_{0} \times_{\operatorname{Spec}(k)} S, \operatorname{pr}_{2}, p_{1}, p_{2}, \text { id }\right)
$$

and $\mathcal{F}^{[0]}:=\mathcal{F}$, and for $i=1, \ldots, n$ define inductively a modification $\left(\mathcal{C}^{[i]}, \pi^{[i]}, s_{1}^{[i]}\right.$, $\left.s_{2}^{[i]}, h^{[i]}\right)$ of $\left(\tilde{C}_{0}, p_{1}, p_{2}\right)$ over $S$ together with a locally free $\mathcal{O}_{\mathcal{C}^{[i]}}$-module $\mathcal{F}^{[i]}$ and an isomorphism $\left(s_{2}^{[i]}\right)^{*} \mathcal{F} \mathcal{F}^{[i]} \stackrel{\sim}{\rightarrow} F_{i}$ as follows:

Assume that $\left(\mathcal{C}^{[i-1]}, \pi^{[i-1]}, s_{1}^{[i-1]}, s_{2}^{[i-1]}, h^{[i-1]}\right)$ together with $\mathcal{F}^{[i-1]}$ and an isomorphism $\left(s_{2}^{[i-1]}\right)^{*} \mathcal{F}^{[i-1]} \stackrel{\sim}{\rightarrow} F_{i-1}$ has already been defined. By Proposition 7.4 , the bf-morphism

$$
\underset{\left(L_{i-1}, \lambda_{i-1}\right)}{\stackrel{\swarrow_{i-1}^{\otimes}}{\longrightarrow}} F_{i-1}
$$

induces a canonical simple modification $\left(\mathcal{C}^{[i]}, f^{[i]}, \pi^{[i]}, s_{2}^{[i]}\right)$ of $\left(\mathcal{C}^{[i-1]}, \pi^{[i-1]}, s_{2}^{[i-1]}\right)$

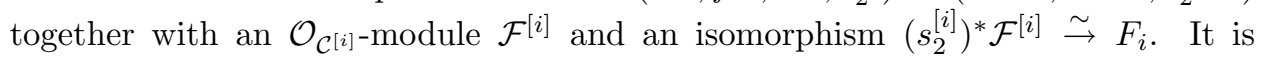
clear that there exists a unique section $s_{1}^{[i]}$ of $\pi^{[i]}$ such that $s_{1}^{[i-1]}=f^{[i]} \circ s_{1}^{[i]}$. Let $h^{[i]}:=h^{[i-1]} \circ f^{[i]}$. 
Analogously, we define for $i=0, \ldots, n$ a modification $\left(\mathcal{C}^{(i)}, \pi^{(i)}, s_{1}^{(i)}, s_{2}^{(i)}, h^{(i)}\right)$ of $\left(\tilde{C}_{0}, p_{1}, p_{2}\right)$ over $S$ together with a locally free $\mathcal{O}_{\mathcal{C}^{(i)}}$-module $\mathcal{F}^{(i)}$ and an isomorphism $\left(s_{1}^{(i)}\right)^{*} \mathcal{F}^{(i)} \stackrel{\sim}{\longrightarrow} E_{i}$ by setting

$$
\left(\mathcal{C}^{(0)}, \pi^{(0)}, s_{1}^{(0)}, s_{2}^{(0)}, h^{(0)}\right):=\left(\mathcal{C}^{[n]}, \pi^{[n]}, s_{1}^{[n]}, s_{2}^{[n]}, h^{[n]}\right)
$$

and repeatedly applying Proposition 7.4 with the role of $s_{1}$ and $s_{2}$ interchanged where we now use the bf-morphism $\left(M_{i-1}, \mu_{i-1}, E_{i} \rightarrow E_{i-1}, M_{i-1} \otimes E_{i} \leftarrow E_{i-1}, i-\right.$ 1) instead of $\left(L_{i-1}, \lambda_{i-1}, F_{i} \rightarrow F_{i-1}, L_{i-1} \otimes F_{i} \leftarrow F_{i-1}, i-1\right)$ for the induction step.

Finally, let

$$
\begin{aligned}
\left(\mathcal{C}, \pi, s_{1}, s_{2}, h\right) & :=\left(\mathcal{C}^{(n)}, \pi^{(n)}, s_{1}^{(n)}, s_{2}^{(n)}, h^{(n)}\right), \\
\mathcal{E} & :=\mathcal{F}^{(n)}, \\
\varphi & : \quad s_{1}^{*} \mathcal{E} \stackrel{\sim}{\rightarrow} E_{n} \stackrel{\sim}{\rightarrow} F_{n} \stackrel{\sim}{\rightarrow} s_{2}^{*} \mathcal{E} .
\end{aligned}
$$

This completes the construction.

Lemma 9.7. The tuple $\left(\mathcal{C}, \pi, s_{1}, s_{2}, h ; \mathcal{E}, \varphi\right)$ constructed in 9.6 is a Gieseker vector bundle data on $\left(\tilde{C}_{0}, p_{1}, p_{2}\right)$ over $S$.

Proof. It is clear by construction that the diagram

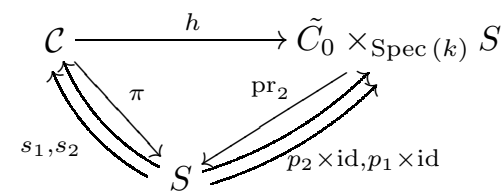

is commutative, that $\pi$ is flat, $h$ is proper and finitely presented and that the $s_{i}$ are sections of $\pi$. Since furthermore Construction 9.6 commutes with base-change, we may assume that $S=\operatorname{Spec}(K)$ for some field $K$.

Let $X$ be a curve over $K, \pi: X \rightarrow \operatorname{Spec}(K)$ the structure morphism and $x_{0} \in X(K)$. Let $\mathcal{G}$ be a locally free $\mathcal{O}_{X}$-module of rank $n$ and let $G:=\mathcal{G}\left[x_{0}\right]$. Let

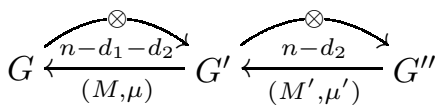

be a sequence of bf-morphisms of $K$-vectorspaces with $\mu=\mu^{\prime}=0$ and such that

$$
\operatorname{im}\left(G \rightarrow \mathcal{M} \otimes G^{\prime} \rightarrow \mathcal{M} \otimes \mathcal{M}^{\prime} \otimes G^{\prime \prime}\right)=\operatorname{im}\left(\mathcal{M} \otimes G^{\prime} \rightarrow \mathcal{M} \otimes \mathcal{M}^{\prime} \otimes G^{\prime \prime}\right)
$$

and

$$
\operatorname{im}\left(G \leftarrow G^{\prime}\right)=\operatorname{im}\left(G \leftarrow G^{\prime} \leftarrow G^{\prime \prime}\right)
$$

Using Proposition 7.4 we get a simple modification $\left(X^{\prime}, f, \pi^{\prime}, x_{1}\right)$ of $\left(X, \pi, x_{0}\right)$ and an $\mathcal{O}_{X^{\prime}}$-module $\mathcal{G}^{\prime}$ together with an isomorphism $\mathcal{G}^{\prime}\left[x_{1}^{\prime}\right] \stackrel{\sim}{\rightarrow} G^{\prime}$. A second application of 7.4 yields a simple modification $\left(X^{\prime \prime}, f^{\prime}, \pi^{\prime \prime}, x_{2}\right)$ of $\left(X^{\prime}, \pi^{\prime}, x_{1}\right)$ and an $\mathcal{O}_{X^{\prime \prime}}$-module $\mathcal{G}^{\prime \prime}$. Thus we have the following situation:
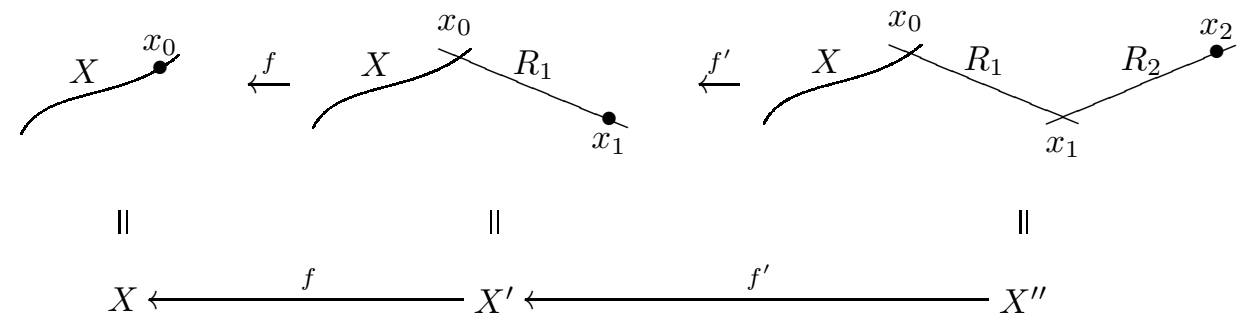
and it is easy to see (by going through Construction 7.9 for this case) that the restriction of $\mathcal{G}^{\prime \prime}$ to the chain of rational curves $R_{1} \cup R_{2}$ is strictly standard. This consideration shows that the restriction of $\mathcal{E}$ to the chains of rational curves $R^{\prime}:=$ $h^{-1}\left(p_{1}\right)$ and $R^{\prime \prime}:=h^{-1}\left(p_{2}\right)$ is strictly standard.

To prove the admissibility of $\left.\mathcal{E}\right|_{R^{\prime}}$, we have to show that $H^{0}\left(R^{\prime},\left.\mathcal{E}\right|_{R^{\prime}}\left(-s_{1}-p_{1}\right)\right)=$ 0 (cf. [3.3). For this let $R_{i}^{\prime}:=\left(h^{(i)}\right)^{-1}\left(p_{1}\right)$ for $i=1, \ldots, n$ and let

$$
R^{\prime}=R_{n}^{\prime} \stackrel{f^{(n)}}{\longrightarrow} R_{n-1}^{\prime} \stackrel{f^{(n-1)}}{\longrightarrow} \cdot \quad \text {. } \quad \stackrel{f^{(2)}}{\longrightarrow} R_{1}^{\prime}
$$

be the successive simple modifications intervening in Construction 9.6. If all $R_{i}^{\prime}$ are reduced to a point, there is nothing to show. Otherwise there exists an $m \in[1, n]$ such that $R_{m}^{\prime} \cong \mathbb{P}^{1}$. By construction we have $f_{*}^{(i)}\left(\mathcal{F}^{(i)}\left(-s_{1}^{(i)}\right)\right)=\mathcal{F}^{(i-1)}\left(-s_{1}^{(i-1)}\right)$ for $i=1, \ldots, n$. It follows that

$$
H^{0}\left(R^{\prime},\left.\mathcal{E}\right|_{R^{\prime}}\left(-s_{1}-p_{1}\right)\right)=H^{0}\left(R_{1}^{\prime},\left.\mathcal{F}^{(m)}\right|_{R_{m}^{\prime}}\left(-s_{1}^{(m)}-p_{1}\right)\right)
$$

which is zero, since $\left.\mathcal{F}^{(m)}\right|_{R_{m}^{\prime}}$ is strictly standard. Analogously, one shows the admissibility of $\left.\mathcal{E}\right|_{R^{\prime \prime}}$.

Now let $R$ be the chain of rational curves which is induced from $R^{\prime}$ and $R^{\prime \prime}$ by identifying the points $s_{1}$ and $s_{2}$ and let $\mathcal{E}_{R}$ be the $\mathcal{O}_{R^{-}}$-module induced by $\left.\mathcal{E}\right|_{R^{\prime}}$, $\left.\mathcal{E}\right|_{R^{\prime \prime}}$ and $\varphi:\left.\left.\mathcal{E}\right|_{R^{\prime}}\left[s_{1}\right] \stackrel{\sim}{\rightarrow} \mathcal{E}\right|_{R^{\prime \prime}}\left[s_{2}\right]$. We have to show that $\mathcal{E}_{R}$ is admissible. This is clear if either $R^{\prime}$ or $R^{\prime \prime}$ is a chain of length zero, so we may assume that both are of length at least one. In the last part of the proof of Lemma 9.3 we have seen that the image of $H^{0}\left(R^{\prime},\left.\mathcal{E}\right|_{R^{\prime}}\left(-p_{1}\right)\right)$ (resp. of $H^{0}\left(R^{\prime \prime},\left.\mathcal{E}\right|_{R^{\prime \prime}}\left(-p_{2}\right)\right)$ in $\left.\mathcal{E}\right|_{R^{\prime}}\left[s_{1}\right]$ (resp. in $\left.\left.\mathcal{E}\right|_{R^{\prime \prime}}\left[s_{2}\right]\right)$ is $\operatorname{ker}\left(E_{0} \leftarrow E_{n}\right)\left(\operatorname{resp} . \operatorname{ker}\left(F_{n} \rightarrow F_{0}\right)\right)$. Since by definition of a generalized isomorphism we have $\varphi\left(\operatorname{ker}\left(E_{0} \leftarrow E_{n}\right)\right) \cap \operatorname{ker}\left(F_{n} \rightarrow F_{0}\right)=0$, it follows that $H^{0}\left(R, \mathcal{E}_{R}\left(-p_{1}-p_{2}\right)\right)=0$ as required.

Proof of Theorem 9.5. By Lemma 9.7 Construction 9.6 is well defined. By Proposition 7.4 it is clear that Construction 9.6 is inverse to Construction 9.2

\section{Relationship With THE STACK OF TORSION FREE SHEAVES}

Let $\operatorname{TFS}(C / B)$ be the algebraic $B$-stack which parametrizes relatively torsionfree sheaves of rank $n$ on $C$ over $B$ (cf. [F]) and let $\operatorname{TFS}\left(C_{0} / B_{0}\right)$ be its special fibre (the $B_{0}$-stack which parametrizes torsion-free sheaves of rank $n$ on $C_{0}$ ). The stack $\operatorname{TFS}(C / B)$ is known to be singular; its local structure has been studied in F]. If $S$ is a $B$-scheme and $\left(h: \mathcal{C} \rightarrow C \times{ }_{B} S, \mathcal{E}\right)$ a Gieseker vector bundle on $C$ over $S$, then the push-forward $h_{*} \mathcal{E}$ is relatively torsion-free (cf. [3.7). Therefore we obtain a morphism $\operatorname{GVB}(C / B) \rightarrow \operatorname{TFS}(C / B)$ which specializes to $\operatorname{GVB}\left(C_{0} / B_{0}\right) \rightarrow$ $\operatorname{TFS}\left(C_{0} / B_{0}\right)$. Denoting, as before, by $\mathcal{E}_{\text {univ }}$ the universal vector bundle on $\tilde{C}_{0} \times$ $\operatorname{VB}\left(\tilde{C}_{0} / B_{0}\right)$, there is also a morphism $\operatorname{Grass}_{n}\left(p_{1}^{*} \mathcal{E}_{\text {univ }} \oplus p_{2}^{*} \mathcal{E}_{\text {univ }}\right) \rightarrow \operatorname{TFS}\left(C_{0} / B_{0}\right)$ which is defined as follows (cf. section 6 of [Se2]): Let $S$ be a $k$-scheme, let $\mathcal{E}$ be a locally free $\mathcal{O}_{\tilde{C}_{0} \times S}$-module of rank $n$ and let $p_{1}^{*} \mathcal{E} \oplus p_{2}^{*} \mathcal{E} \longrightarrow Q$ be an epimorphism of $\mathcal{O}_{S}$-modules, where $Q$ is locally free of rank $n$. To these data we associate a relatively torsion-free $\mathcal{O}_{C_{0} \times S}$-module $\mathcal{F}$ by setting

$$
\mathcal{F}:=\operatorname{ker}\left(r_{*} \mathcal{E} \rightarrow p_{*}\left(p_{1}^{*} \mathcal{E} \oplus p_{2}^{*} \mathcal{E}\right) \rightarrow p_{*} Q\right),
$$

where $r: \tilde{C}_{0} \times S \rightarrow C_{0} \times S$ is induced by the normalization morphism and $p: S \rightarrow$ $C_{0} \times S$ is the section induced by the singular point $p \in C_{0}(k)$. Finally, by section 
10 of [K1] there is a canonical morphism

$$
\operatorname{KGl}_{n}\left(p_{1}^{*} \mathcal{E}_{\text {univ }}, p_{2}^{*} \mathcal{E}_{\text {univ }}\right) \rightarrow \operatorname{Grass}_{n}\left(p_{1}^{*} \mathcal{E}_{\text {univ }} \oplus p_{2}^{*} \mathcal{E}_{\text {univ }}\right) .
$$

Proposition 10.1. The following diagram is commutative:

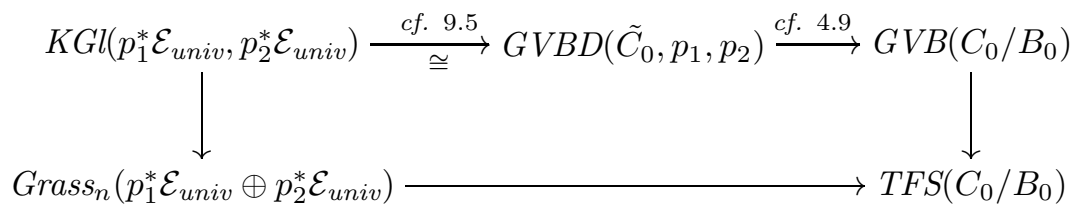

Proof. Let $S$ be a $k$-scheme. Let

$$
\begin{aligned}
& \tilde{C} \stackrel{f}{\longrightarrow} \tilde{C}_{0} \times S \\
& \tilde{\pi} \prod_{S} s_{1}, s_{2} \\
& \text { S }
\end{aligned}
$$

be a modification of $\left(\tilde{C}_{0}, p_{1}, p_{2}\right)$ over $S$ and let $\left(\tilde{\mathcal{E}}, \varphi: s_{1}^{*} \tilde{\mathcal{E}} \stackrel{\sim}{\rightarrow} s_{2}^{*} \tilde{\mathcal{E}}\right)$ be a locally free $\mathcal{O}_{\tilde{\mathcal{C}}}$-module of rank $n$ which is amissible for $\left(\tilde{\mathcal{C}}, \tilde{\pi}, s_{1}, s_{2}, f\right)$. Recall the definition of $f_{\bullet} \mathcal{E}$ from Construction 9.1. We have $f_{\bullet} \mathcal{E}=\left(f_{*} \tilde{\mathcal{E}}\left(-s_{1}-s_{2}\right)\right)\left(p_{1}+p_{2}\right)$. Let $\left(h: \mathcal{C} \rightarrow C_{0} \times S, \mathcal{E}\right.$ ) be the Gieseker vector bundle on $C_{0}$ over $S$ associated to the Gieseker vektor bundle data $\left(\tilde{\mathcal{C}}, \tilde{\pi}, s_{1}, s_{2}, f, \mathcal{E}, \varphi\right)$. Then we have a commutative diagram:

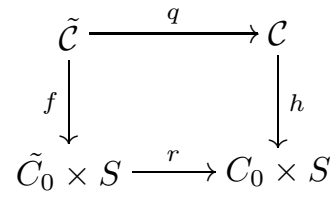

where $q$ is the clutching morphism which maps the sections $s_{1}$ and $s_{2}$ of $\tilde{\pi}$ onto a section $s$ of $\pi$, which meets $\mathcal{C}$ in the singular locus of $\pi$. Let $s_{1}+s_{2}: S \amalg S \rightarrow \tilde{\mathcal{C}}$ and $p_{1}+p_{2}: S \amalg S \rightarrow \tilde{C}_{0} \times S$ be the morphisms induced by the sections $s_{1}, s_{2}$ and $p_{1}, p_{2}$. Similarly as in 7.2 , we have a commutative diagram with exact rows:

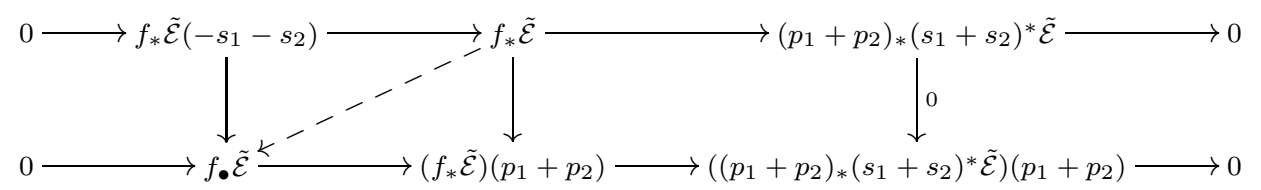

which induces the arrow $f_{*} \tilde{\mathcal{E}} \rightarrow f_{\bullet} \tilde{\mathcal{E}}$. Thus we obtain the following diagram with exact rows:

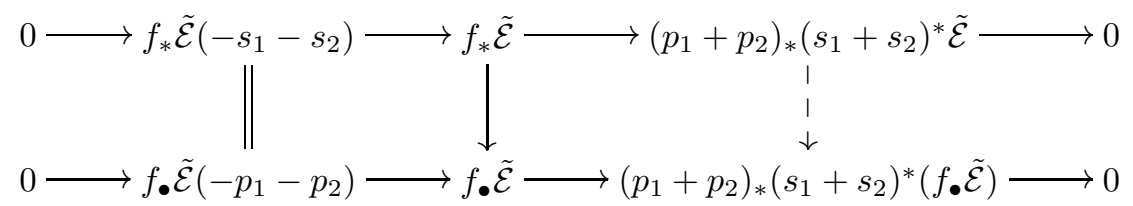

and in particular a canonical morphism $\bar{\alpha}: s_{1}^{*} \tilde{\mathcal{E}} \oplus s_{2}^{*} \tilde{\mathcal{E}} \rightarrow p_{1}^{*}\left(f_{\bullet} \tilde{\mathcal{E}}\right) \oplus p_{2}^{*}\left(f_{\bullet} \tilde{\mathcal{E}}\right)$. Let $Q$ be the cokernel of the composed morphism

$$
s_{1}^{*} \tilde{\mathcal{E}} \stackrel{(\mathrm{id}, \varphi)}{\longrightarrow} s_{1}^{*} \tilde{\mathcal{E}} \oplus s_{2}^{*} \tilde{\mathcal{E}} \stackrel{\bar{\alpha}}{\longrightarrow} p_{1}^{*}\left(f_{\bullet} \tilde{\mathcal{E}}\right) \oplus p_{2}^{*}(f . \tilde{\mathcal{E}})
$$


Then the data consisting in $f_{\bullet} \tilde{\mathcal{E}}$ together with the quotient map

$$
p_{1}^{*}\left(f_{\bullet} \tilde{\mathcal{E}}\right) \oplus p_{2}^{*}\left(f_{\bullet} \tilde{\mathcal{E}}\right) \longrightarrow Q
$$

is the image under

$$
\operatorname{GVBD}\left(\tilde{C}_{0}, p_{1}, p_{2}\right) \stackrel{\sim}{\rightarrow} \operatorname{KGl}\left(p_{1}^{*} \mathcal{E}_{\text {univ }}, p_{2}^{*} \mathcal{E}_{\text {univ }}\right) \rightarrow \operatorname{Grass}_{n}\left(p_{1}^{*} \mathcal{E}_{\text {univ }} \oplus p_{2}^{*} \mathcal{E}_{\text {univ }}\right)
$$

of the Gieseker vector bundle data $\left(\tilde{\mathcal{C}}, \tilde{\pi}, s_{1}, s_{2}, f, \mathcal{E}, \varphi\right)$. Therefore it suffices to show that there exists a canonical exact sequence of $\mathcal{O}_{\tilde{C}_{0} \times S}$-modules as follows:

$$
0 \longrightarrow h_{*} \mathcal{E} \longrightarrow r_{*}(f \cdot \tilde{\mathcal{E}}) \stackrel{\beta}{\longrightarrow} p_{*} Q \longrightarrow 0
$$

where $\beta: r_{*}\left(f_{\bullet} \tilde{\mathcal{E}}\right) \rightarrow p_{*} Q$ is the composed map

$$
r_{*}\left(f_{\bullet} \tilde{\mathcal{E}}\right) \rightarrow r_{*}\left(p_{1}+p_{2}\right)_{*}\left(p_{1}+p_{2}\right)^{*}\left(f_{\bullet} \tilde{\mathcal{E}}\right)=p_{*}\left(p_{1}^{*}\left(f_{\bullet} \tilde{\mathcal{E}}\right) \oplus p_{2}^{*}\left(f_{\bullet} \tilde{\mathcal{E}}\right)\right) \rightarrow p_{*} Q .
$$

For abbreviation we set $E_{n}:=s_{1}^{*} \tilde{\mathcal{E}}, F_{n}:=s_{2}^{*} \tilde{\mathcal{E}}, E_{0}:=p_{1}^{*} f_{\bullet} \tilde{\mathcal{E}}, F_{0}:=p_{2}^{*} f_{\bullet} \tilde{\mathcal{E}}$. Applying $r_{*}$ to the diagram $(*)$ gives the exact diagram

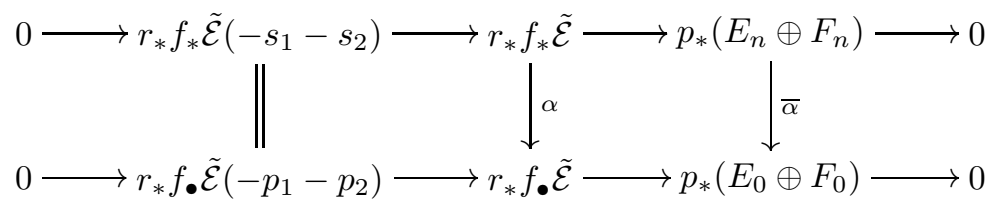

We also have the following exact diagram:

$(* * *)$

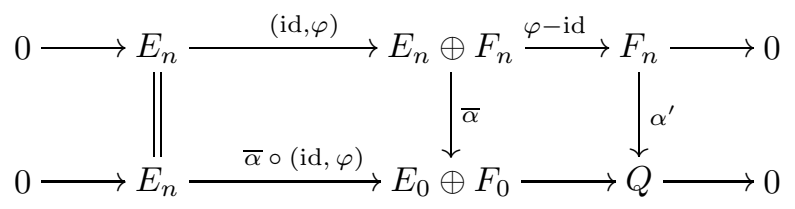

where $\alpha^{\prime}$ is the morphism induced by $\bar{\alpha}$. From diagrams $(* *)$ and $(* * *)$ it follows that the following diagram commutes:

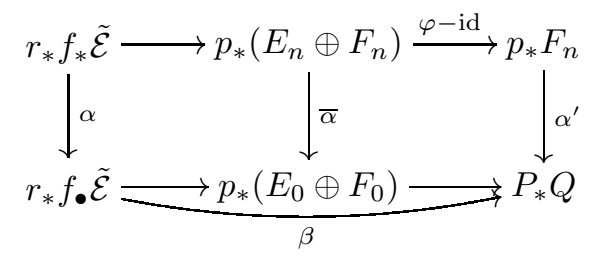

Therefore the right square in the following diagram $(\dagger \dagger)$ commutes:

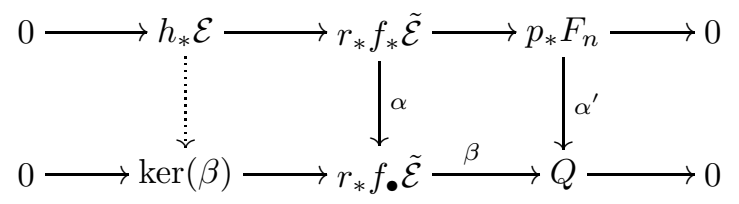

The upper row in $(\dagger \dagger)$ is exact, since it comes from the exact sequence

$$
0 \longrightarrow \mathcal{E} \longrightarrow f_{*} \tilde{\mathcal{E}} \longrightarrow s_{*} F_{n} \longrightarrow 0
$$

by applying the functor $h_{*}$. Furthermore, it follows from $(* *)$ and $(* * *)$ that in diagram $(\dagger)$ the horizontal arrows induce isomorphisms $\operatorname{ker}(\alpha) \stackrel{\sim}{\rightarrow} \operatorname{ker}\left(\alpha^{\prime}\right)$ and 
$\operatorname{coker}(\alpha) \stackrel{\sim}{\rightarrow} \operatorname{coker}\left(\alpha^{\prime}\right)$. Therefore the dotted arrow in $(\dagger \dagger)$ is an isomorphism as required.

Remark 10.2. For $n>1$ the square in Proposition 10.1 is not cartesian. This can be seen as follows. Let $K$ be an algebraically closed field and let $\left(\mathcal{G}, \mathcal{G}\left[p_{1}\right] \oplus \mathcal{G}\left[p_{2}\right] \rightarrow Q\right)$ be a $K$-valued point of $\operatorname{Grass}_{n}\left(p_{1}^{*} \mathcal{E}_{\text {univ }} \oplus p_{2}^{*} \mathcal{E}_{\text {univ }}\right)$ such that $\operatorname{rk}\left(\mathcal{G}\left[p_{1}\right] \rightarrow Q\right) \neq n \neq$ $\operatorname{rk}\left(\mathcal{G}\left[p_{2}\right] \rightarrow Q\right)$ (here we need $\left.n>1\right)$. Let $r: \tilde{C}_{0} \otimes_{k} K \rightarrow C_{0} \otimes_{k} K$ be the normalization morphism and let $\mathcal{F}:=\operatorname{ker}\left(r_{*} \mathcal{G} \rightarrow Q\right)$ be the torsion free sheaf on $C_{0} \otimes_{k} K$, associated to $\left(\mathcal{G}, \mathcal{G}\left[p_{1}\right] \oplus \mathcal{G}\left[p_{2}\right] \rightarrow Q\right)$. By Lemma 2.1 (3) in [Sun] there exists a $K$ valued point $\left(\mathcal{G}^{\prime}, \mathcal{G}^{\prime}\left[p_{1}\right] \oplus \mathcal{G}^{\prime}\left[p_{2}\right] \rightarrow Q^{\prime}\right)$ of $\operatorname{Grass}_{n}\left(p_{1}^{*} \mathcal{E}_{\text {univ }} \oplus p_{2}^{*} \mathcal{E}_{\text {univ }}\right)$ such that $\operatorname{ker}\left(r_{*} \mathcal{G}^{\prime} \rightarrow Q\right) \cong \mathcal{F}$ and $\operatorname{rk}\left(\mathcal{G}^{\prime}\left[p_{1}\right] \rightarrow Q^{\prime}\right)=n$. But then Proposition 10.1 in [K1] tells us that the respective fibres of

$$
\operatorname{KGl}\left(p_{1}^{*} \mathcal{E}_{\text {univ }}, p_{2}^{*} \mathcal{E}_{\text {univ }}\right) \rightarrow \operatorname{Grass}_{n}\left(p_{1}^{*} \mathcal{E}_{\text {univ }} \oplus p_{2}^{*} \mathcal{E}_{\text {univ }}\right)
$$

over the points $\left(\mathcal{G}, \mathcal{G}\left[p_{1}\right] \oplus \mathcal{G}\left[p_{2}\right] \rightarrow Q\right)$ and $\left(\mathcal{G}^{\prime}, \mathcal{G}^{\prime}\left[p_{1}\right] \oplus \mathcal{G}^{\prime}\left[p_{2}\right] \rightarrow Q^{\prime}\right)$ are not isomorphic (as would be the case, if the square in Proposition 10.1 was cartesian).

\section{REFERENCES}

[B] N. Bourbaki: Algèbre, Eléments de Math, Hermann, Paris (1947-59). MR 9:406e

[Bho] U. Bhosle: Generalised parabolic bundles and applications to torsionfree sheaves on nodal curves. Ark. Mat. 30 (1992), no. 2, 187-215. MR.95g:14022

[B-L] A. Beauville and Y. Laszlo: Un lemme de descent, C. R. Acad. Sci. Paris, t. 320, Série I, p. 335-340, 1995. MR96a:14049

[D] P. Deligne: Le lemme de Gabber. Séminaire sur les pinceaux arithmetiques: La conjecture de Mordell, Astérisque 127 (1985), 131-150, Société mathématique de France. MR:87h:14017

[F] G. Faltings: Moduli-stacks for bundles on semistable curves. Math. Ann. 304 (1996), 489-515. MR $97 \mathrm{~d}: 14016$

[G] D. Gieseker: A Degeneration of the Moduli Space of Stable Bundles, J. Differential Geometry 19 (1984), 173-206. MR85j:14014

[EGA I] A. Grothendieck, J. Dieudonné: Éléments de géometrie algébrique I. Die Grundlehren der mathematischen Wissenschaften in Einzeldarstellungen, Band 166, Springer-Verlag 1971. MR:55:5621

[EGA II] A. Grothendieck, J. Dieudonné: Éléments de géometrie algébrique II. Publications Mathématiques IHES, $N^{o}$ 8, (1961). MR29:1208

[EGA III] A. Grothendieck, J. Dieudonné: Éléments de géometrie algébrique III. Publications Mathématiques IHES, $N^{o}$ s 11, 17, $(1961,1963)$. MR29:1209 MR29:1210

[EGA IV] A. Grothendieck, J. Dieudonné: Éléments de géometrie algébrique IV. Publications Mathématiques IHES, $N^{o}$ S 20, 24, 28, 32 (1964, 1965, 1966, 1967). MR 30:3885 MR 33:7330 MR $36: 178$

[SGA1] A. Grothendieck, M. Raynaud: Séminaire de géometrie algébrique du Bois Marie 1960-61. Springer Lecture Notes 224 (1971). MR50:7129

[K1] I. Kausz: A Modular Compactification of the General Linear Group, Documenta Math. 5 (2000) 553-594. MR2002a:14056

[K2] I. Kausz: A canonical decomposition of generalised theta functions on the moduli stack of Gieseker vector bundles. Preprint (April 2003).

[Kn] F. F. Knudsen: The projectivity of the moduli space of stable curves, II: The stacks $M_{g, n}$. Math. Scand. 52 (1983), 161-199. MR85d:14038a

[Ma] H. Matsumura: Commutative ring theory. Cambridge studies in advanced mathematics 8. Cambridge University Texts (1989). MR90i:13001

[Mi] J.S. Milne: Étale Cohomology. Princeton University Press (1980). MR81j:14002

[LM] G. Laumon, L. Moret-Bailly: Champs algebriques. Ergebnisse der Mathematik un ihrer Grenzgebiete, Volume 39. Springer 2000. MR2001f:14006 
[NR] M.S. Narasimhan,T.R. Ramadas: Factorisation of generalised theta functions I. Invent. Math. 114 (1993), no. 3, 565-623. MR.94i:14017

[NS] D.S. Nagaraj and C.S. Seshadri: Degenerations of the moduli spaces of vector bundles on curves II. Proc. Indian Acad. Sci. Math. Sci. 109 (1999), no 2, 165-201. MR2000c:14046

[N] P.E. Newstead: Introduction to moduli problems and orbit spaces. Lectures held at Tata Institute of Fundamental Research, Bombay. Springer Verlag, 1978. MR $81 \mathrm{k}: 14002$

[Se1] C.S. Seshadri: Fibrés vectoriels sur les courbes algébriques. Notes written by J.-M. Drezet from a course at the École Normale Supérieure, June 1980. Astérisque, 96. Société Mathématique de France, Paris, 1982. MR85b:14023

[Se2] C.S. Seshadri: Degenerations of the moduli spaces of vector bundles on curves. School on Algebraic Geometry (Trieste, 1999), 205-265, ICTP Lect. Notes, 1, Abdus Salam Int. Cent. Theoret. Phys., Trieste, 2000. MR2001i:14044

[So] C. Sorger: La formule de Verlinde. Séminaire Bourbaki, Vol. 1994/95. Astérisque No. 237, (1996), Exp. No. 794, 3, 87-114. MR98f:14009

[Sun] Xiaotao Sun: Degeneration of moduli spaces and generalized theta functions. J. Algebraic Geom. 9 (2000), no. 3, 459-527. MR2001h:14040

[T] M. Teixidor i Bigas: Compactifications of moduli spaces of (semi)stable bundles on singular curves: two points of view. Collect. Math. 49 (1998), no. 2-3, 527-548. MR 2000e:14050

NWF I - Mathematik, Universität Regensburg, 93040 Regensburg, Germany

E-mail address: ivan.kausz@mathematik.uni-regensburg.de 